\title{
The Preliminary Design of the SHARCS Rotor Blade
}

by

Travis Mikjaniec, B. Eng.

\author{
A thesis submitted to \\ The Faculty of Graduate Studies and Research \\ in partial fulfilment of \\ the degree requirements of \\ Master of Applied Science
}

Ottawa-Carleton Institute for

Mechanical and Aerospace Engineering

Department of Mechanical and Aerospace Engineering

Carleton University

Ottawa, Ontario, Canada

April, 2006

Copyright (C)

2006 - Travis Mikjaniec 


$\begin{array}{ll}\begin{array}{l}\text { Library and } \\ \text { Archives Canada }\end{array} & \begin{array}{l}\text { Bibliothèque et } \\ \text { Archives Canada }\end{array} \\ \begin{array}{l}\text { Published Heritage } \\ \text { Branch }\end{array} & \begin{array}{l}\text { Direction du } \\ \text { Patrimoine de l'édition }\end{array} \\ \begin{array}{l}\text { 395 Wellington Street } \\ \text { Ottawa ON K1A 0N4 } \\ \text { Canada }\end{array} & \begin{array}{l}\text { 395, rue Wellington } \\ \text { Ottawa ON K1A ON4 } \\ \text { Canada }\end{array}\end{array}$

Your file Votre référence ISBN: 978-0-494-16463-1 Our file Notre référence ISBN: 978-0-494-16463-1

NOTICE:

The author has granted a nonexclusive license allowing Library and Archives Canada to reproduce, publish, archive, preserve, conserve, communicate to the public by telecommunication or on the Internet, loan, distribute and sell theses worldwide, for commercial or noncommercial purposes, in microform, paper, electronic and/or any other formats.

The author retains copyright ownership and moral rights in this thesis. Neither the thesis nor substantial extracts from it may be printed or otherwise reproduced without the author's permission.
AVIS:

L'auteur a accordé une licence non exclusive permettant à la Bibliothèque et Archives Canada de reproduire, publier, archiver, sauvegarder, conserver, transmettre au public par télécommunication ou par l'Internet, prêter, distribuer et vendre des thèses partout dans le monde, à des fins commerciales ou autres, sur support microforme, papier, électronique et/ou autres formats.

L'auteur conserve la propriété du droit d'auteur et des droits moraux qui protège cette thèse. $\mathrm{Ni}$ la thèse ni des extraits substantiels de celle-ci ne doivent être imprimés ou autrement reproduits sans son autorisation.
In compliance with the Canadian

Privacy Act some supporting forms may have been removed from this thesis.

While these forms may be included in the document page count, their removal does not represent any loss of content from the thesis.
Conformément à la loi canadienne sur la protection de la vie privée, quelques formulaires secondaires ont été enlevés de cette thèse.

Bien que ces formulaires aient inclus dans la pagination, il n'y aura aucun contenu manquant.

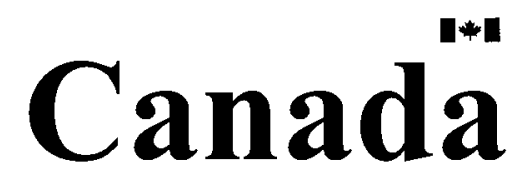




\begin{abstract}
A topic of current rotorcraft research is the development of jet-smooth and quiet rotary flight. To achieve this goal, three rotor blade sub-systems have been developed to reduce the noise and vibrations associated with rotorcraft flight. Testing these systems requires the design and manufacture of a Mach scaled rotor blade with similar rotating natural frequencies as the full scale Messerschmitt-Bölkow-Blohm (MBB) BO 105 rotor blade. The rotor blade and the sub-systems are to be tested in a 4 by 4 metre wind tunnel. This proof of concept wind tunnel rotor blade and the sub-systems are known as SHARCS (Smart Hybrid Active Rotor Control System). In this thesis, a preliminary design and analysis of the SHARCS rotor blade structure is explored.
\end{abstract}

The design of the rotor blade requires the use of principles of rotor blade aerodynamics, mechanics of composites, and structural analysis. Using these principles enables the loads imposed on the blade, its mass, flexural and torsional rigidities, stresses, and the safety factor for a given design to be calculated. The design of the composite lay-up is iterated until a safe structure with rotating natural frequencies within the required range is obtained. To this end, the commercial finite element software MSC.NASTRAN and ANSYS are employed. It is shown using the finite element analysis that the preliminary rotor blade design does indeed satisfy all the requirements that are imposed for this blade at the outset of the project. 


\section{For Mom}




\section{ACKNOWLEDGEMENTS}

I would like to thank my thesis co-supervisors Professor Fred Nitzsche and Professor Choon-Lai Tan. Also, I would like to thank Professor Daniel Feszty for his help in the field of rotorcraft aerodynamics. It is especially important to thank Dr. Mehrdaad Ghorashi for his guidance over the course of this project.

Importantly, without the help of Peter Wierach this project would not have been accomplished. I would like to thank Tony Firmin for his patience and help with ANSYS.

A thank you goes to my fellow Masters of Applied Science students Michael Balajewicz, Marc-Andre Müller, Greg Davis, and Dan Brassard who have contributed to this project in one way or another. A special shout out goes to Jackie Yu (㡼右民) and Phil Genereux, who have given countless hours aiding me in both course and project work, and the honing of my road hockey skills. Finally, a thank you goes to my mom, for being so supportive during my life. 


\section{TABLE OF CONTENTS}

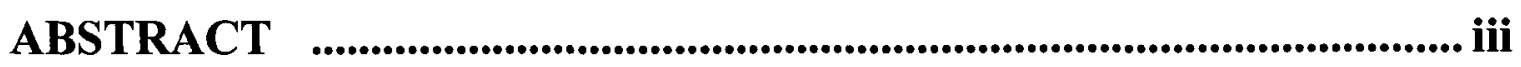

ACKNOWLEDGEMENTS .................................................................... v

TABLE OF CONTENTS ................................................................. vi

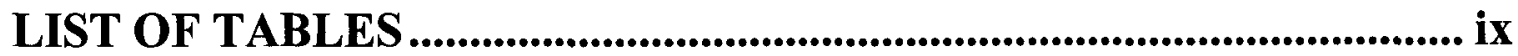

LIST OF FIGURES .......................................................................................... $x$

NOMENCLATURE............................................................................. xiii

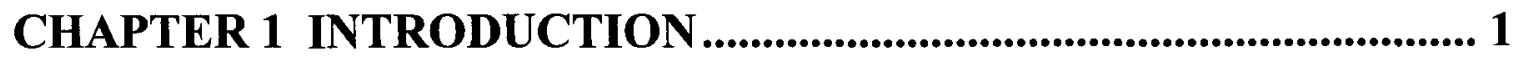

CHAPTER 2 DESIGN REQUIREMENTS......................................... 5

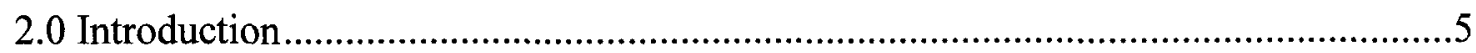

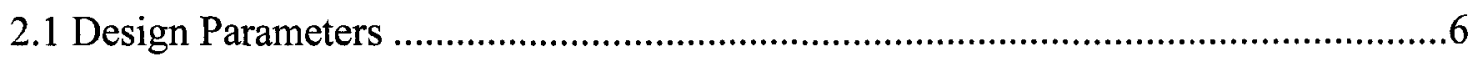

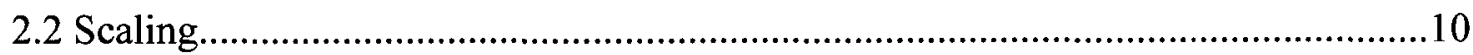

2.3 Required Structural Rigidity .........................................................................12

\section{CHAPTER 3 REVIEW OF COMPOSITE MATERIAL ANALYSIS. 17}

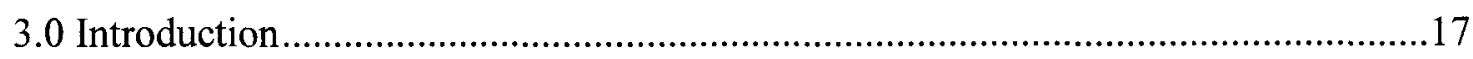

3.1 Classical Laminate Theory ...............................................................................17

3.2 Classical Laminate Theory Simplifications ...........................................................19

3.3 Laminate Theory ……………………………………...............................21

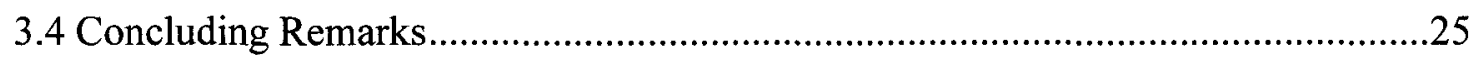

CHAPTER 4 ROTOR BLADE LOADS............................................ 26 


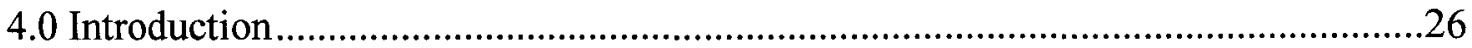

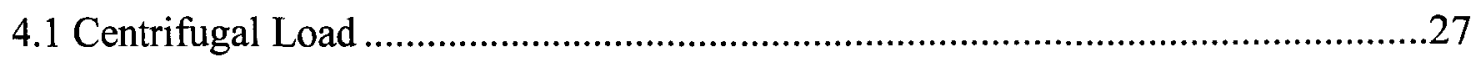

4.2 Angle of Attack

$4.3 \mathrm{Lift}$

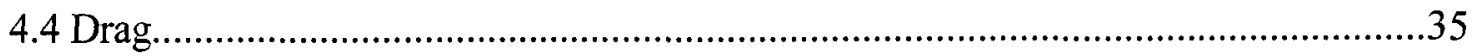

4.5 Flapping and Lead-Lag Bending Moment.........................................................38

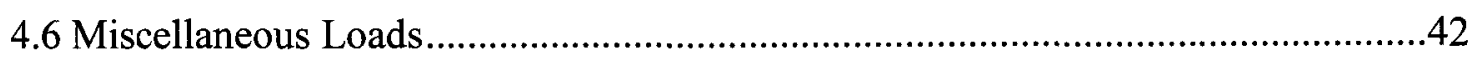

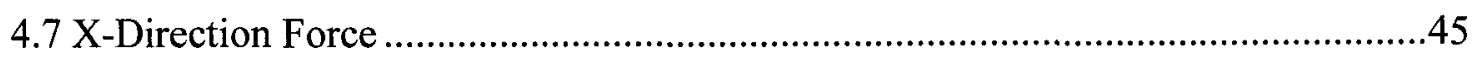

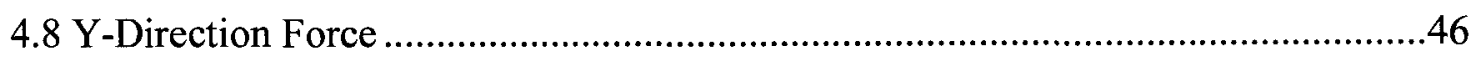

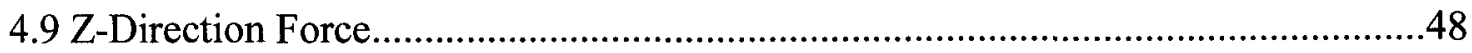

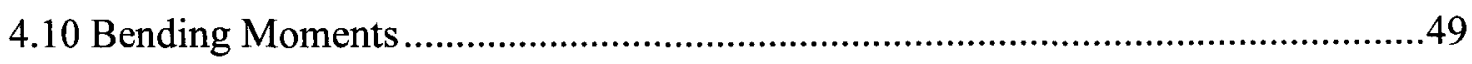

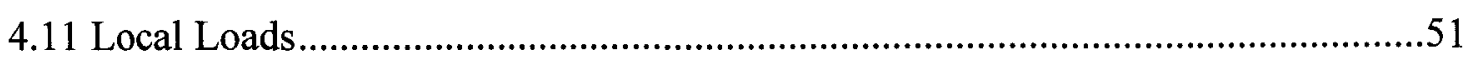

CHAPTER 5 STRUCTURAL ANALYSIS ..........................................52

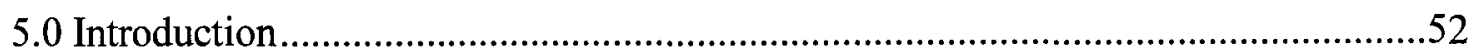

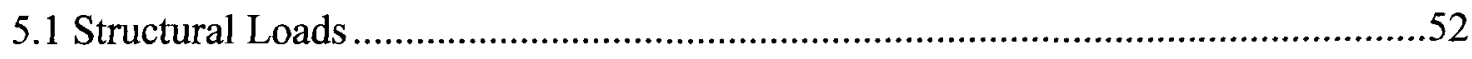

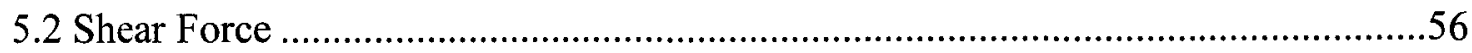

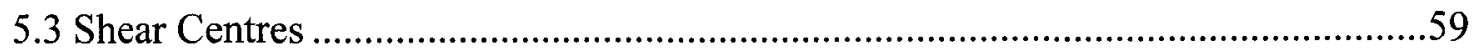

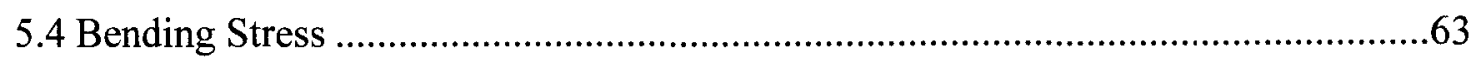

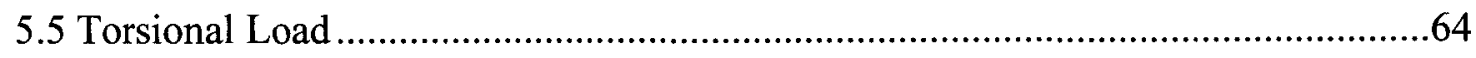

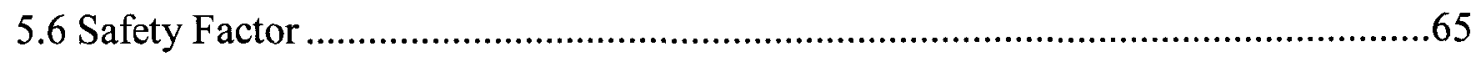

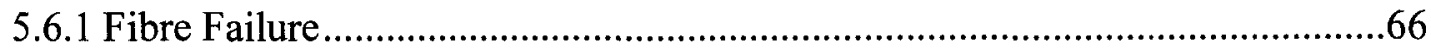

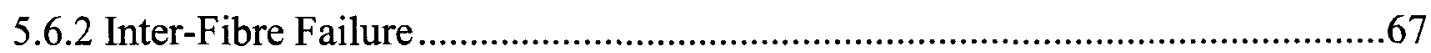

5.6.3 Failure Loci for Inter-Fibre Failure.........................................................

5.6.4 Mathematical Representation of the Failure Banana ..........................................74

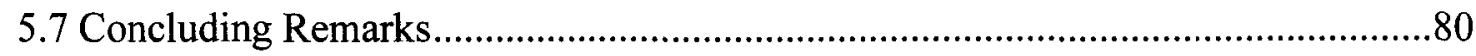

CHAPTER 6 SHARCS ROTOR BLADE DESIGN_............................ 81

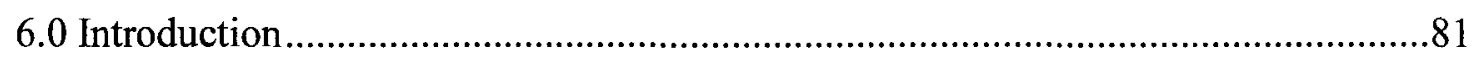

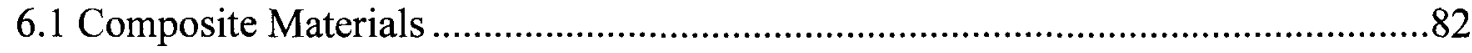




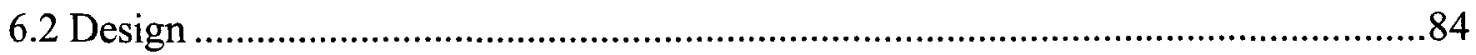

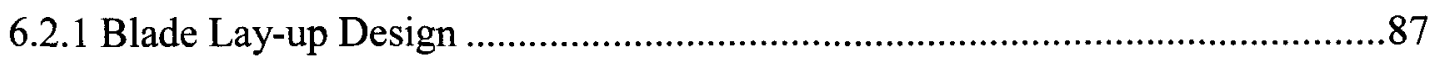

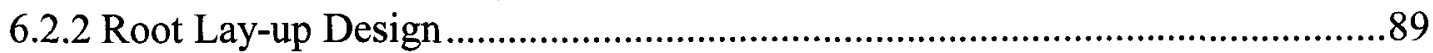

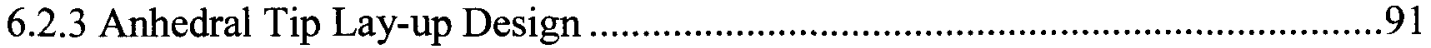

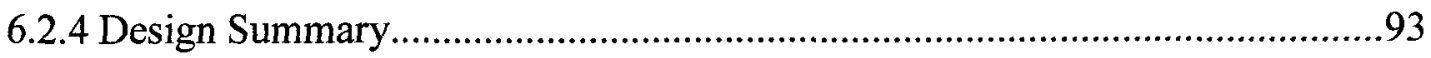

6.3 MSC.Nastran/Patran Finite Element Model Validation .......................................95

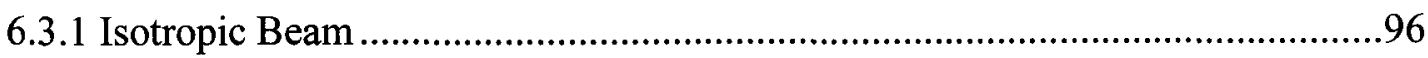

6.3.2 Static Deflection of a Cantilevered Isotropic Beam ...................................97

6.3.3 Non-Rotating Natural Frequencies of an Isotropic Beam...............................99

6.3.4 Composite Beam Static Deflection ...................................................... 102

6.3.5 Composite Beam Natural Frequencies.................................................... 103

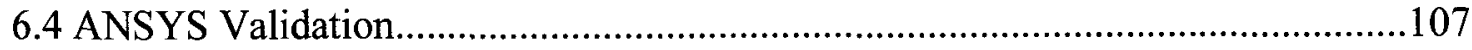

6.5 Composite Rotor Blade Natural Frequencies ..................................................108

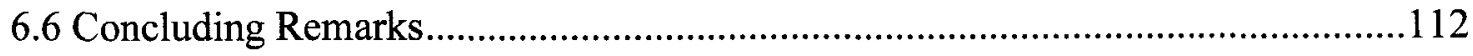

CHAPTER 7 CONCLUSIONS......................................................... 113

REFERENCES.............................................................................. 115

Appendix A: Iterative Design Process Flow Chart......................................A

Appendix B: Drag Polar for NACA 0012 Airfoil [Abbott and Doenhoff

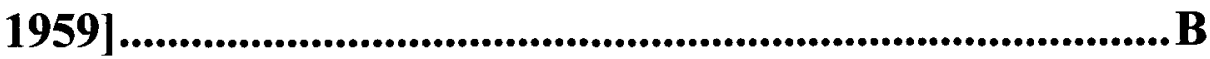

Appendix C: Lift Data for NACA 0012 Airfoil [Abbott and Doenhoff

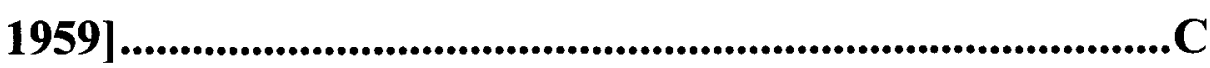

Appendix D: Profile Data for NACA 0012 Airfoil lAbbott and

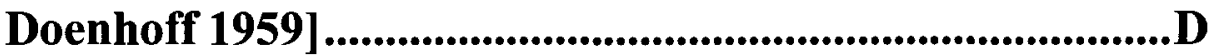

Appendix E: BO 105 Rotor Blade Fan Plot [Anonymous 1993] .............E 


\section{LIST OF TABLES}

Table 2.1: Blade rigidity initial value comparisons $\quad 15$

Table 4.1: Input data for the rotor blade load program 26

Table 6.1: Composite material properties $\quad 83$

Table 6.2: SHARCS main blade lay-up $\quad 88$

Table 6.3: SHARCS root lay-up $\quad 90$

Table 6.4: Anhedral tip lay-up $\quad 93$

Table 6.5: Summary of SHARCS rotor blade lay-up summary 94

Table 6.6: Design versus target stiffness values $\quad 95$

Table 6.7: Isotropic beam properties $\quad 97$

Table 6.8: MSC.Nastran and theoretical static deflection results 98

Table 6.9: MSC.Nastran and theoretical static torsion results 98

Table 6.10: MSC.Nastran and theoretical natural frequencies of the non-rotating $\begin{array}{ll}\text { isotropic beam } & 100\end{array}$

Table 6.11: Composite rotor blade static deflection comparison 103

Table 6.12: Non-dimensional frequency comparison for $\left[0^{\circ} / 90^{\circ} / 90^{\circ} / 0^{\circ}\right]$ graphite/epoxy beam with $\mathrm{L} / \mathrm{h}=15 \quad 105$

Table 6.13: Non-dimensional frequency comparison for $\left[0^{\circ} / 90^{\circ} / 90^{\circ} / 0^{\circ}\right]$ graphite/epoxy beam with $\mathrm{L} / \mathrm{h}=10 \quad 106$

Table 6.14: ANSYS and MSC.Nastran model comparison $\quad 107$

Table 6.15: Articulated rotating natural frequencies for SHARCS rotor blade 110

Table 6.16: SHARCS rotating natural frequencies and required frequency range 111 


\section{LIST OF FIGURES}

Figure 1.1: SHARCS rotor blade sub-systems 2

Figure 1.2: 2-D schematic of blade vortex interaction [Leishman 2000] 3

Figure 2.1: Example articulated rotor $\quad 7$

Figure 2.2: Flapping and lead-lag motion [Leishman 2000] 7

Figure 2.3: Schematic diagram of the rotor blade 9

Figure 2.4: Twist distribution 9

Figure 2.5: NACA 0012 profile $\quad 10$

Figure 3.1: Example laminates [Kollar 2003] 18

Figure 3.2: Lamina co-ordinate system $\quad 21$

Figure 3.3: Angle lamina $\quad 22$

Figure 4.1: Force equilibrium about the rotor blade flapping hinge [Leishman 2000] 28

Figure 4.2: Centrifugal force along the span of the rotor blade 29

Figure 4.3: Incident velocities and aerodynamic forces for a rotor blade element $\begin{array}{ll}\text { [Leishman 2000] } & 30\end{array}$

Figure 4.4: Variations of pitch $(\theta)$, induced $(\phi)$ and angle of attack $(\alpha)$ along the span of the rotor blade

Figure 4.5: SHARCS actual and theoretical differential lift distribution along the span of the rotor blade $\quad 34$

Figure 4.6: Variation of lift reaction force along the span of the rotor blade 35

Figure 4.7: Force equilibrium about the lead-lag hinge [Leishman 2000] 36

Figure 4.8: Differential drag force along the span of the rotor blade 37

Figure 4.9: Drag reaction force along the span of the rotor blade 38

Figure 4.10: Force diagram for the flapping moment balance of a rotor blade [Leishman 2000] 39

Figure 4.11: Distribution of the flapping moment along the span of the rotor blade $\quad 40$

Figure 4.12: Lead-lag moment along the span of the rotor blade $\quad 42$ 
Figure 4.13: Rotor blade static forces

Figure 4.14: Resultant shear force along the span of the blade due to static loads 44

Figure 4.15: Bending moment distribution along the blade due to static loads 45

Figure 4.16: Variation of the force $Q_{x}$ along the span wise direction of the blade $\quad 46$

Figure 4.17: Static and dynamic transverse shear force along the span of the rotor blade $\quad 47$

Figure 4.18: Maximum transverse shear force along the span of the rotor blade $\quad 48$

Figure 4.19: Z-axis shear force distribution along the span of the rotor blade $\quad 49$

Figure 4.20: Bending moment about the y-axis along the span of the rotor blade $\quad 50$

Figure 4.21: Flapping and static moments about the z-axis along the span of the rotor $\begin{array}{ll}\text { blade } & 50\end{array}$

Figure 4.22: Maximum magnitude z-axis moment along the span of the rotor blade 51

Figure 5.1: Schematic rotor blade cross-section with C-spar 53

Figure 5.2: Schematic homogeneous rotor blade cross-section $\quad 54$

Figure 5.3: Structure centre-line $\quad 56$

Figure 5.4: Schematic of rotor blade leading edge discretization 59

Figure 5.5: Three inter-fibre failure modes [Puck 2002] 68

Figure 5.6: Debonding effect of mode C [Puck 2002] 69

Figure 5.7: Failure locus for zero fibre stress [Möller 2004] 70

Figure 5.8: Idealized and actual failure ellipse in the parallel and shear stress axes $\quad 72$

Figure 5.9: Fibre failure ellipse in the parallel and perpendicular stress plane $\quad 73$

Figure 5.10: Failure Banana [Möller 2004] 73

Figure 5.11: $d^{*}$ ellipse $\quad 75$

Figure 6.1: Simplified spar $\quad 85$

Figure 6.2: Schematic of SHARCS rotor blade sections $\quad 87$

Figure 6.3: Main blade section of the SHARCS rotor blade $\quad 87$

Figure 6.4: Blade root lay-up of the SHARCS rotor blade $\quad 91$

Figure 6.5: Anhedral tip lay-up for the SHARCS rotor blade 93 
Figure 6.6: MSC.NASTRAN isotropic beam model

Figure 6.7: Flap frequency response diagram for articulated blade to random unit input applied to the rotor blade tip

Figure 6.8: Lead-lag frequency response diagram for articulated blade to random unit input applied to the rotor blade tip

Figure 6.9: Torsional frequency response diagram for articulated blade to random unit input applied to the rotor blade tip

Figure 6.10: MSC.Nastran composite beam model

Figure 6.11: Computational time versus number of layers for ANSYS composite shell elements [ANSYS help file, 2005]

Figure 6.12: ANSYS model of the SHARCS rotor blade 109

Figure 6.13: SHARCS rotor blade fanplot $\quad 110$

Figure 6.14: SHARCS $1^{\text {st }}$ elastic flapping mode $\quad 111$

Figure 6.15: SHARCS $2^{\text {nd }}$ elastic flapping mode 111

Figure 6.16: SHARCS $1^{\text {st }}$ elastic lead-lag mode 111

Figure 6.17: SHARCS $1^{\text {st }}$ torsion mode $\quad 112$

xii 


\section{NOMENCLATURE}

$\begin{array}{ll}A & \text { cross-sectional area } \\ A_{i j} & \text { sub-matrix relating direct stresses to direct strains } \\ a & \text { speed of sound; loci constant } \\ B & \text { tip loss coefficient } \\ B_{i j} & \text { sub-matrix relating direct stresses to shear strains } \\ b & \text { span; loci constant } \\ \text { BVI } & \text { blade vortex interaction } \\ c & \text { chord length; loci constant } \\ C_{i j} & \text { stiffness matrix } \\ C_{D} & \text { coefficient of drag } \\ C_{D 0} & \text { parasitic drag coefficient } \\ C_{D 2} & \text { induced drag coefficient } \\ d^{*} & \text { force; yield strength } \\ C_{L \alpha}, \frac{d C_{L}}{d \alpha} & \text { lift curve slope } \\ D_{i j} & \text { sub-matrix relating shear stresses to shear strains } \\ d & \text { loci constant } \\ & \end{array}$

xiii 


\begin{tabular}{|c|c|}
\hline FEA & finite element analysis \\
\hline FEM & finite element model \\
\hline$G$ & shear modulus \\
\hline$g$ & acceleration due to gravity; straight-line vector \\
\hline GJ & torsional rigidity \\
\hline$h$ & height of blade element above radius reference line; beam height \\
\hline$I$ & second moment of area \\
\hline$I_{0}$ & polar moment of inertia \\
\hline$J$ & torsion constant \\
\hline$j$ & safety factor \\
\hline$L$ & length \\
\hline$M$ & Mach number; moment \\
\hline$m$ & mass \\
\hline$m_{x}$ & mass per unit length \\
\hline$n$ & number of lamina; mode number \\
\hline$Q$ & shear force \\
\hline$q$ & shear flow \\
\hline$q_{o}$ & constant shear flow \\
\hline$R$ & radius \\
\hline$r$ & radial co-ordinate \\
\hline$r_{0}$ & hub radius \\
\hline$r_{t}$ & perpendicular distance from the moment centre to tangent of the skin \\
\hline
\end{tabular}


first moment of area

$S_{i j}$

compliance matrix

$s$

mid-thickness cross-section perimeter co-ordinate; vector representing the stress state

$T_{i j} \quad$ transformation matrix

$t$

thickness

$V_{T I P} \quad$ tip speed

$V_{f} \quad$ volume fibre fraction

$v_{i} \quad$ induced velocity

W applied load

$x \quad \mathrm{x}$ co-ordinate

$y \quad$ y co-ordinate

$\hat{y} \quad$ distance from neutral axis in y co-ordinate

$z \quad$ number of blades; $z$ co-ordinate

$\hat{z} \quad$ distance from neutral axis in $\mathrm{z}$ co-ordinate

$\alpha \quad$ angle of attack; fibre angle

$\beta \quad$ coning angle; Timoshenko beam coefficient

$\beta L \quad$ frequency equation root

$\delta \quad$ deflection

$\varepsilon \quad$ strain

$\phi \quad$ induced angle

Xv 


$\begin{array}{ll}\lambda & \text { Lock number } \\ \gamma & \text { shear strain } \\ \nu & \text { Poisson's ratio } \\ \theta & \text { pitch angle; cross-section twist angle } \\ \rho & \text { beam density } \\ \rho_{\infty} & \text { air density } \\ \sigma & \text { stress } \\ \tau & \text { shear stress } \\ \Omega & \text { rotational speed } \\ \omega & \text { natural frequency } \\ \zeta & \text { lead-lag angle }\end{array}$

\section{Subscripts}

1,2,3 parallel, perpendicular in-plane, perpendicular out-of-plane to fibre direction

$x, y, z \quad$ span-wise, perpendicular, and chord-wise axes

bending

c closed section; compressive

CF $\quad$ centrifugal

drag

xvi 


\begin{tabular}{|c|c|}
\hline eff & effective \\
\hline$F F$ & fibre failure \\
\hline$g$ & global \\
\hline hom & homogeneous \\
\hline$i$ & station along the profile \\
\hline$I F F$ & inter-fibre failure \\
\hline$L$ & lift \\
\hline$M$ & shear centre; moment \\
\hline maint & maintenance \\
\hline $\max$ & maximum \\
\hline Non-Dim & non-dimensionalized \\
\hline$p$ & centre of mass \\
\hline per-rev & cycles per revolution \\
\hline res & resultant \\
\hline stat & static \\
\hline$T$ & torque \\
\hline$t$ & tension \\
\hline
\end{tabular}

xvii 


\section{CHAPTER 1 INTRODUCTION}

Noise and vibration reduction are two areas of active research for rotorcraft. Noise caused by blade-vortex interaction (BVI) has many drawbacks. It limits the areas of operation of helicopters, decreases the stealth of military vehicles, and affects the comfort of passengers. Vibrations from the rotor blades cause fatigue cracking of the engineering components of the rotorcraft, thus requiring additional maintenance; they can also cause fatigue of the pilots and passengers. In addition, excessive vibrations limit the forward flight speed of the rotorcraft. Both vibration and noise are produced as a result of the complex aerodynamics associated with rotary-wing flight. Various attempts have been made to test rotor blades with noise or vibration reduction systems in wind tunnels [see, e.g., Chopra and Bernhard (1998, 2002), Chopra and Koratkar (2001), Chopra and Shen $(2003,2004)]$. A problem with these approaches is that reduction of either the noise or the vibration results in an increase of the other.

A novel approach to reducing both noise and vibration has been developed by Professor Nitzsche in collaboration with an industrial partner, known as the SHARCS (Smart Hybrid Active Rotor Control System), shown in Figure 1.1. The SHARCS system is comprised of three sub-systems: the smart spring, the flap, and the anhedral tip. The 
smart spring is a variable stiffness system used in place of the rigid pitch link, which changes the torsional boundary condition; this reduces the vibrations transmitted to the fuselage.

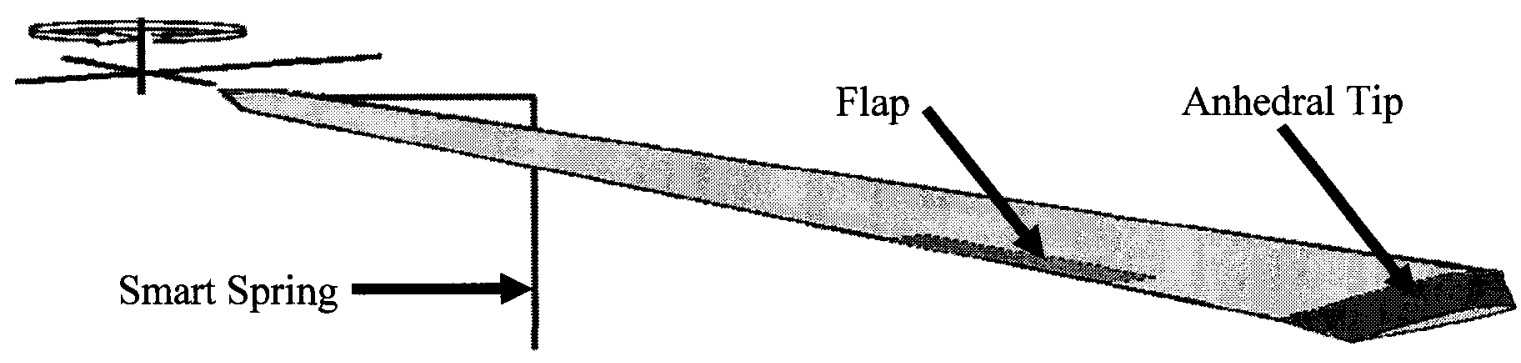

Figure 1.1: SHARCS rotor blade sub-systems

Flap systems have been shown to reduce the rotor blade vibrations [Chopra and Koratkar (2002)] by momentarily reducing the angle of attack. The benefit of this is that the blade can avoid dynamic stall, and the associated vibrations. To alleviate the noise caused by BVI, the anhedral tip pivots about the chord axis to change the tip vortex path. By changing the position of the vortex, the distance between the vortex and the following rotor blade can be increased (Figure 1.2), thereby reducing the generated noise. 


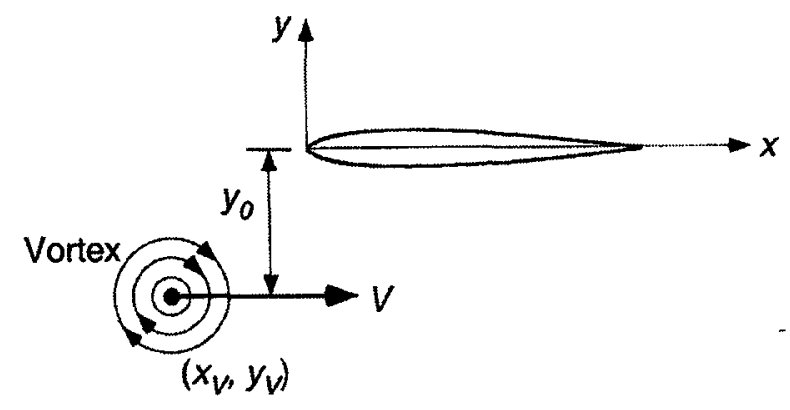

Figure 1.2: 2-D schematic of blade vortex interaction [Leishman 2000]

This thesis is concerned with the preliminary design of a SHARCS rotor blade, carried out in collaboration with an industrial partner, and the Deutsches Zentrum für Luft- und Raumfahrt (DLR) in Germany. This rotor blade would serve as a platform for testing the SHARCS sub-systems in a 4 by $4 \mathrm{~m}$ wind tunnel facility. To ensure the noise generated by the rotor blade could be compared to that of a full-scale rotorcraft, the blade was required to be Mach scaled. The blade is also required to have similar rotating natural frequencies as the existing MBB BO 105 rotor blade; this is in order to compare the effectiveness of the vibration reduction systems with the existing vibration data obtained for that rotor blade, which has become a standard reference within the rotorcraft industry.

The organization of this thesis will be as follows. In the next chapter, the design requirements of the SHARCS rotor blade, the scaling calculations, and the estimation of the required flexural and torsional rigidity values will be discussed. A brief review of the mechanics of composite laminates will be provided in Chapter 3. Chapter 4 explores the various loads the rotor blade would experience during its lifespan. These loads include 
the aerodynamic forces calculated using the Blade Element Momentum Theory (BEMT), and the inertial and static loads. With these loads, the procedures for the structural analysis of the rotor blade, and calculation of the safety factor of the structure are described in Chapter 5. The outcome of the preliminary design of the SHARCS rotor blade will be presented in Chapter 6 , as will the validation and creation of a finite element model with the commercial software MSC.Nastran and ANSYS. The purpose of the finite element analysis was the determination of the rotating natural frequencies, to ensure that the design conforms to the requirements. The main conclusions from this work will then be summarized in Chapter 7 . 


\section{CHAPTER 2 DESIGN REQUIREMENTS}

\subsection{Introduction}

The requirements for the SHARCS rotor blade were set to ensure the rotor blade would provide an acceptable platform to demonstrate the vibration and noise reduction subsystems in a wind tunnel test set-up. This requires the SHARCS rotor blade be Mach scaled, aeroelastic and Lock number similar to existing full-scale rotor blades. To Mach scale a rotor blade, the Mach number at the tip of the rotor blade is made equal to that of the full-scale rotor blade. As the BVI noise generated by a rotor blade strongly depends on the tip Mach number, Mach scaling would ensure that the noise generated is within the range of full-scale rotor blades. Aeroelastic similarity was set to ensure that the vibration reduction systems would reduce the primary vibrations that are experienced by full-scale rotor blades. The Lock number is a non-dimensional parameter representing the ratio of the aerodynamic force to the inertia forces acting on the rotor blade. A similar Lock number to that of full-scale rotor blades would therefore ensure that an acceptable ratio of these forces exists. Thus the excitation forces for rotor blade vibrations would then correspond in scale to those that exist for full-scale rotor blades. 


\subsection{Design Parameters}

Due to the 4 by $4 \mathrm{~m}$ size of the available wind tunnel, there are geometric limitations on the size of the rotor blade. The span of the rotor blade has to be chosen such as to avoid wall effects, yet must be long enough to accurately represent a rotor blade. Also the chord length has to be set so as to maintain a representative rotor blade aspect ratio. The industrial partner determined these geometric requirements.

Based on the dimensions of the rotor blade hub at the wind tunnel, the hub radius and number of rotor blades requirements were obtained. Specific details about the hub have yet to be provided, but was assumed to be made of titanium. The rotor hub also determined the requirement for an articulated rotor blade (example, Figure 2.1). The rotor blade boundary conditions are thus pinned for flapping and lead-lag bending, but fixed for torsion due to the rigid pitch link. Flapping and lead-lag motion is schematically shown in Figure 2.2. 


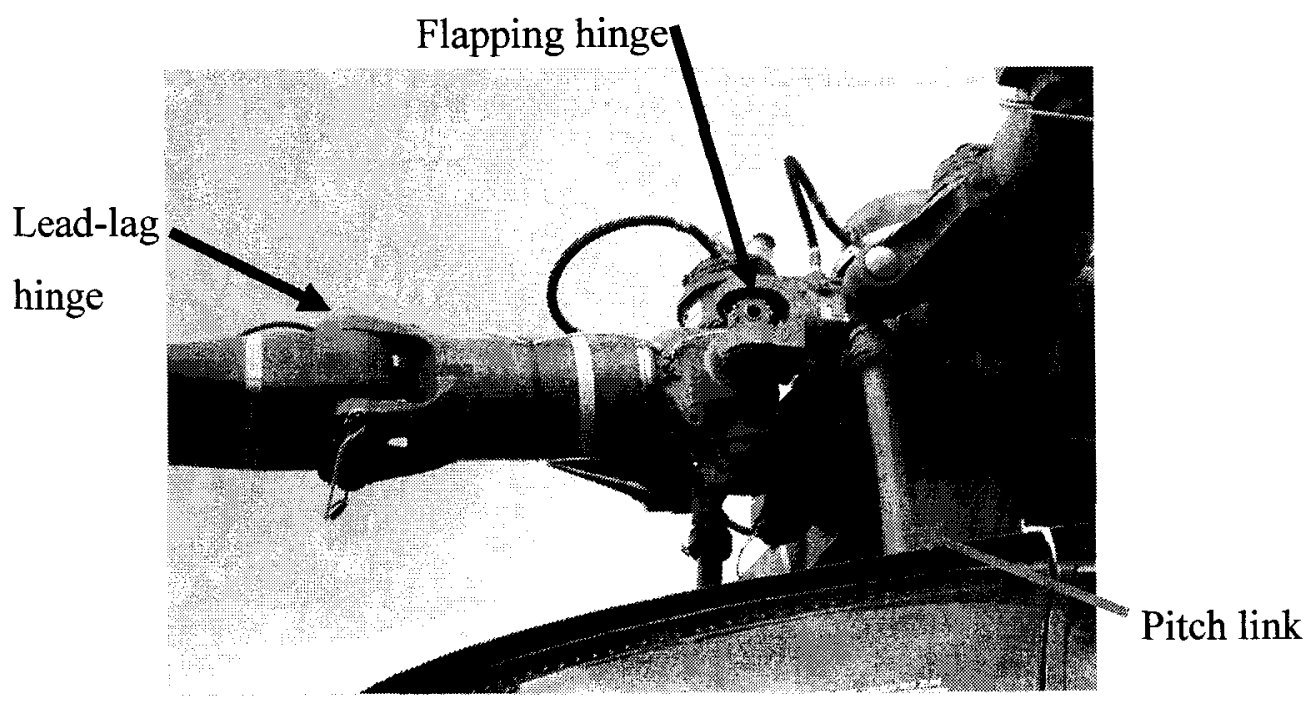

Figure 2.1: Example articulated rotor

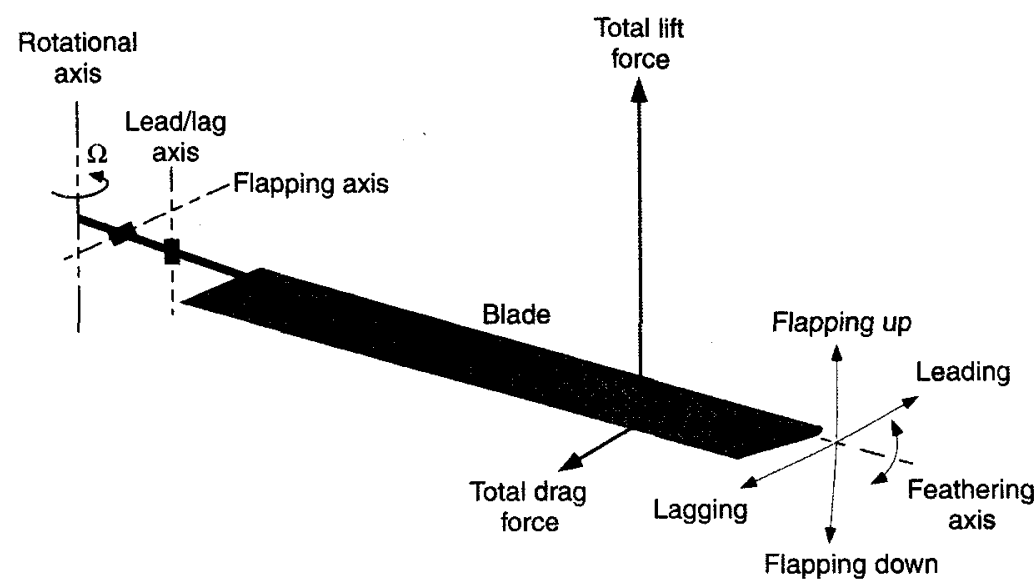

Figure 2.2: Flapping and lead-lag motion [Leishman 2000]

The industrial partner specified a further set of requirements so the SHARCS blade would be comparable to its existing rotor blades. They required a specific twist profile, the specific ranges for aeroelastic similarity, and composite material construction. A 
summary of the values for the key design parameters and other requirements is given below.

- $\quad \operatorname{Span}=1 \mathrm{~m}$

- $\quad$ Chord $=7.53 \mathrm{~cm}$

- Composite construction

- Specified twist distribution

- Articulated rotor

- NACA 0012 airfoil

- Rotating elastic natural frequencies:

$$
\begin{aligned}
& \left.\circ 1^{\text {st }} \text { flapping }=2.5-2.8 \text { cycles per revolution [per-rev }\right] \\
& \circ 2^{\text {nd }} \text { flapping }=4.2-4.7 \text { per-rev } \\
& \circ 1^{\text {st }} \text { lead-lag }=4.5-5.5 \text { per-rev } \\
& \circ \quad 1^{\text {st }} \text { torsion }=5.5-6 \text { per-rev }
\end{aligned}
$$

- $\quad$ Lock number $=5-6$

- $\quad$ Tip speed =0.6 Mach number

- Centre of mass $\approx 1 / 4$-chord (for aeroelastic stability)

- $\quad$ Number of blades $=4$

- Hub radius $=0.126 \mathrm{~m}$

Figure 2.3 shows a schematic of the rotor blade to be designed, while Figure 2.4 illustrates the required twist distribution and Figure 2.5 shows the NACA 0012 profile. 


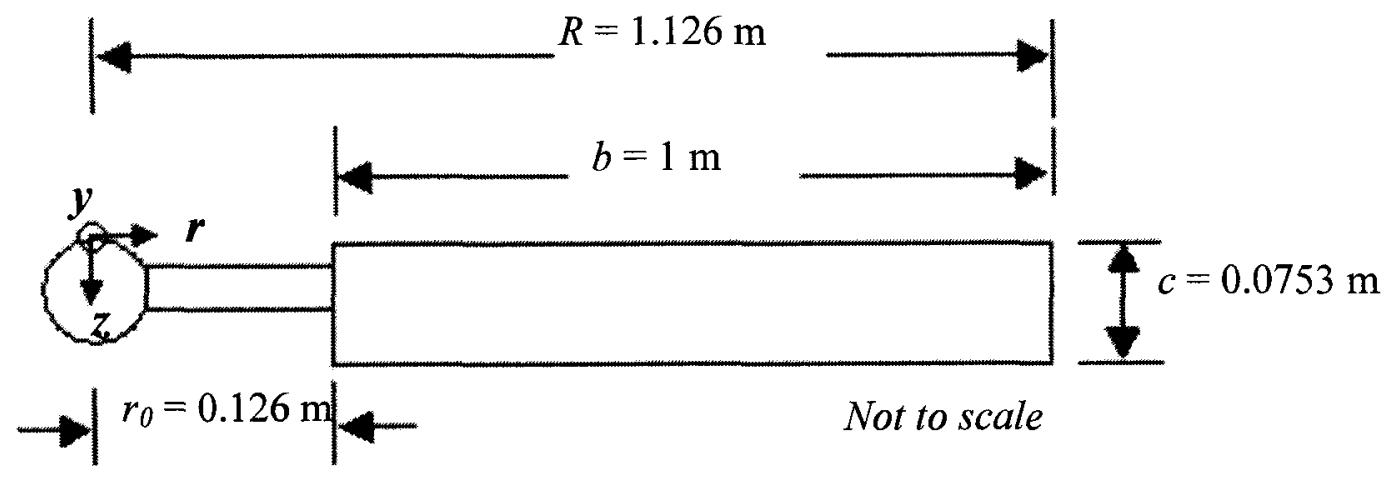

Figure 2.3: Schematic diagram of the rotor blade

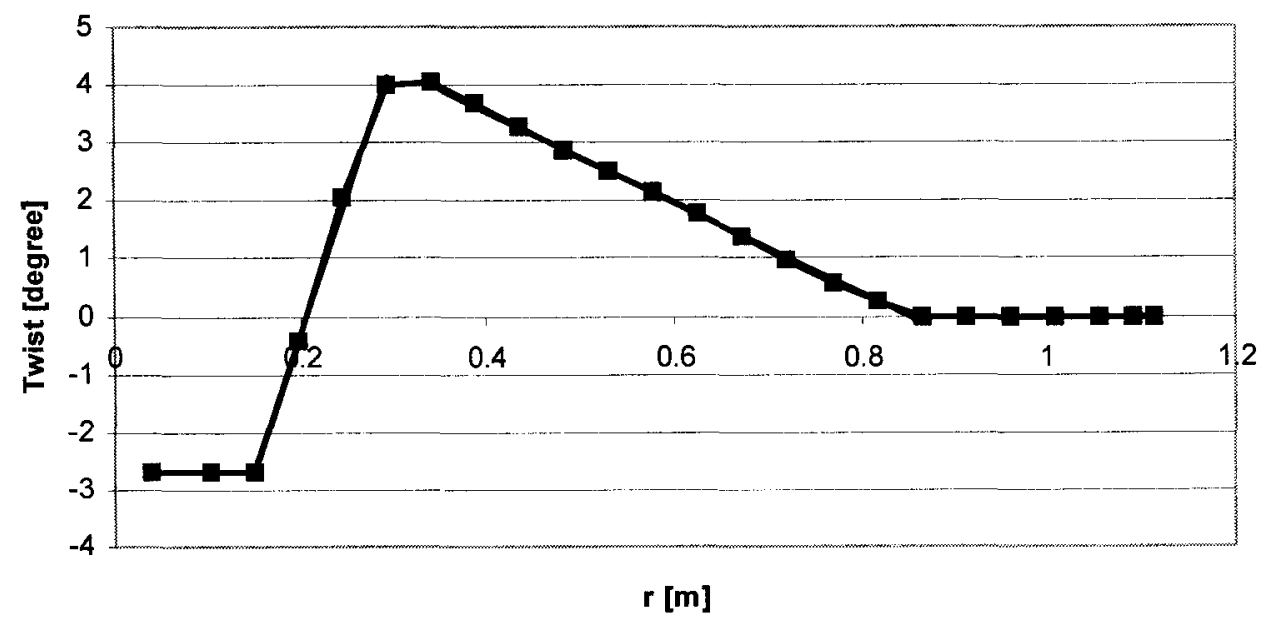

Figure 2.4: Twist distribution 


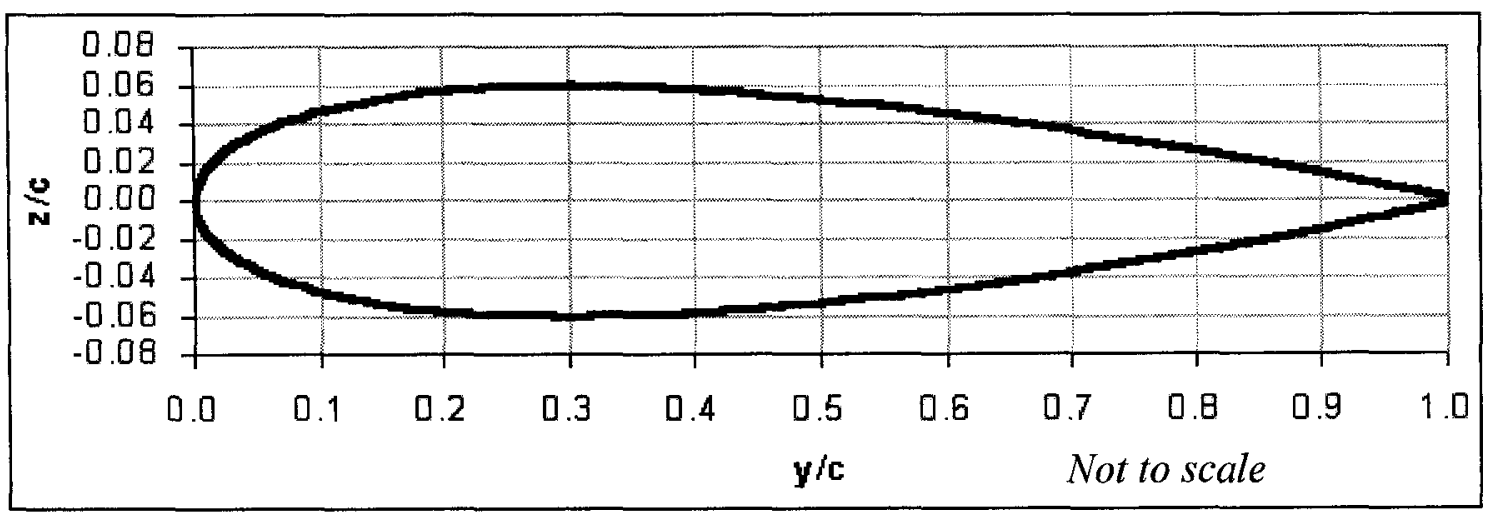

Figure 2.5: NACA 0012 profile

\subsection{Scaling}

Due to the dimensions of the wind tunnel, the SHARCS blade requires a $1 \mathrm{~m}$ span. Knowing the required tip speed of Mach number $=0.6$, the rotational velocity was obtained from these two requirements by the following:

$$
\Omega_{\text {SHARCS }}=\frac{V_{T I P}}{R}=\frac{M \cdot a}{b+r_{o}}=\frac{0.6 * 340}{1.126}=181 \mathrm{rad} / \mathrm{s}
$$

where: $a$ is the speed of sound, and $\Omega_{S H A R C S}$ is the rotational velocity;

However, with this velocity of rotation, it was found using an iterative process illustrated with a flow chart in Appendix A and discussed along this report that the loads exceeded the strength of the final design and the acceptable centrifugal load on the rotor hub. It was, therefore, reduced to $150 \mathrm{rad} / \mathrm{s}$. The result was that the tip speed was no longer 0.6 Mach, but: 


$$
M=\frac{\Omega_{\text {SHARCS }}\left(b+r_{o}\right)}{a}=\frac{150 * 1.126}{340}=0.5
$$

This tip Mach number was deemed to be acceptable for BVI noise generation; therefore the design proceeded using the rotational velocity of $150 \mathrm{rad} / \mathrm{s}(1433 \mathrm{rpm})$.

Next, the initial weight estimation was performed to allow the load calculations to be carried out. From the expression for the Lock number, $\lambda, \mathrm{Eq}(2.3)$, which relates the aerodynamic forces to that of the inertia forces, the weight estimate can be obtained. It's important that this ratio is representative of a full-scale blade to ensure that neither force dominate the characteristics of the SHARCS rotor blade. For example, if the Lock number was smaller, that means the aerodynamic forces would have a diminished effect on the characteristics of the SHARCS rotor blade. In Eq. (2.3), $C_{L \alpha}$ is the lift curve slope, $\rho_{\infty}$ is the density of air, and $\mathrm{m}$ is the mass of the rotor blade. Rotor blades usually have a Lock number in the range of 5 to 6 . As an initial guess, the SHARCS rotor blade was assumed to have a Lock number of 5. Using an approximate lift curve slope, $C_{L \alpha}$, of $2 \pi$ for the NACA 0012 (airfoil data in Appendix B, C and D), the blade mass was calculated as,

$$
\lambda=\frac{3 \cdot \rho_{\infty} \cdot C_{L \alpha} \cdot c \cdot R}{m}
$$




$$
m=\frac{3 \cdot \rho_{\infty} \cdot C_{L \alpha} \cdot c \cdot R}{\lambda}=\frac{3 \cdot 1.225 \cdot 2 \pi \cdot 0.0753 \cdot 1.126}{5}=0.392 \mathrm{~kg}
$$

After a few design iterations, it was decided that the Lock number should be at most 3.7. This was because the smaller mass of the blade for a Lock number of 5 would not allow for sufficient weight of the actuation system and other components. This decision was consistent with those by Bernhard and Chopra (2002), who had also discussed the construction of a Mach scaled rotor blade with active blade tips. However, in the design presented by those authors a Lock number of only 2.55 was achieved. As a result, they had to reduce the tip Mach number because the centrifugal load on the hub from the increased mass became excessive. Using the Lock number of 3.7 , the total mass of the SHARCS blade was determined to be $0.536 \mathrm{~kg}$.

\subsection{Required Structural Rigidity}

Of primary importance in the design of the SHARCS rotor blade was matching the rotating natural frequencies to those of the design requirements. Designing a rotor blade for specific natural frequencies is required for the reduction of vibrations and to avoid aeroelastic problems (Peters et al. 1983). The required rotating natural frequencies depend on the structural rigidity about each axis. Therefore, in order to proceed with the design, these rigidity values were required. To calculate the corresponding rigidity 
values, preliminary calculations were performed using homogeneous, non-rotating beam equations.

For comparison purposes, these calculations were compared to results from Carleton's rotorcraft group's in-house SMARTROTOR program [Nitzsche 2005], from which the structural rigidities for a 1-D isotropic rotating beam can be estimated. This program couples the structural and aerodynamic effects on a rotor blade, as the complex vibrations of the rotor blade are affected by the aerodynamic forces exerted on the rotor blade, and the aerodynamics of the rotor blade are affected by the motions of the rotor blade. The program also has the capability to estimate the noise generated by the rotor blade. To use this program, the structural rigidities were varied until the correct rotating natural frequencies were obtained. While both these methods only gave estimates for the required beam rigidities ( $E I$ 's and $G J$ ), they provided a good starting point for the iterative design process for their determination.

The preliminary rigidity calculations were performed using the following equations, with the length set equal to the radius of the blade, $\omega_{\text {per-rev }}$ as the given target natural frequency values, $\Omega_{\text {SHARCS }}$ as obtained from Eq. (2.1), $m_{x}$ the mass per unit length of the blade, $L$ the length of the blade, $n$ the mode number, $I_{0}$ the polar moment of inertia, and $\rho$ the blade density. 


$$
\begin{aligned}
& \omega_{\text {SHARCS }}=\omega_{\text {per-rev }} * \Omega_{\text {SHARCS }} \\
& E I=\left(\frac{\omega_{\text {SHARCS }}}{(\beta L)^{2}}\right)^{2} \cdot m_{x} \cdot L^{4} \\
& G J=\left(\omega_{\text {SHARCS }} \cdot \frac{2 L}{(2 n-1) \cdot \pi}\right)^{2} \cdot \rho \cdot I_{0}
\end{aligned}
$$

where $\beta L=3.927$ for the $1^{\text {st }}$ elastic mode and $\beta L=7.069$ for the $2^{\text {nd }}$ elastic mode of the articulated (pinned-free) boundary conditions, using the method discussed in Inman (2001) and de Silva (2000). Hence, the $\beta L$ term represents the roots for the characteristic equation for the bending vibration of beams, which gives the normalized mode shapes and natural frequencies.

Table 2.1 lists the calculated stiffness values; they are compared with the computed ones obtained with the SMARTROTOR program. The 1-D beam used in SMARTROTOR was isotropic; therefore the program is limited in its ability to provide realistic behaviour of a composite blade. It should be noted that the blade stiffnesses in flapping are not identical for the corresponding $1^{\text {st }}$ and $2^{\text {nd }}$ modes because the calculated stiffness values were simply obtained by substituting the rotating blade natural frequencies into the nonrotating beam equations. 
The reason for this is that the natural frequencies increase during rotation, due to the centrifugal load providing a restoring moment to any bending of the rotor blade. This natural frequency increase occurs at different rates for the different modes (see Appendix E). Thus, using the non-rotating equations would provide higher stiffness values as the centrifugal stiffening effect would be taken as a structural stiffness, and the values obtained from the $1^{\text {st }}$ and $2^{\text {nd }}$ modes would not be equal due to the difference in the stiffening rates between the two modes.

Consequently, the flexural rigidity $E I$ values obtained through SMARTROTOR were used in the subsequent steps. Since in SMARTROTOR, the blade stiffening effect due to its rotational speed is taken into account, more accurate results were expected in this case. However, for the torsional rigidity $G J$, the calculated value using Eq. (2.6b) was used because the corresponding SMARTROTOR result was unrealistically large, and could not be attain due to the geometric constraints of the rotor blade. This was attributed to the 1-D element used not being suited to calculating torsional rigidities.

Table 2.1: Blade rigidity initial value comparisons

\begin{tabular}{lcc}
\hline & $\begin{array}{c}\text { Non-Rotating } \\
\text { Beam Formulae }\end{array}$ & $\begin{array}{c}\text { SMARTROTOR } \\
\text { Simulation }\end{array}$ \\
\hline \hline $1^{\text {st }}$ Flap El $\left[\mathrm{Nm}^{2}\right]$ & 602 & 40 \\
$2^{\text {nd }}$ Flap El $\left[\mathrm{Nm}^{2}\right]$ & 162 & 40 \\
$1^{\text {st }}$ Lead-Lag $E l\left[\mathrm{Nm}^{2}\right]$ & 1950 & 1600 \\
$1^{\text {st }}$ Torsion GJ $\left[\mathrm{Nm}^{2}\right]$ & 50 & 650 \\
\hline
\end{tabular}


With the design requirements established and the preliminary flexural and torsional rigidities determined, the iterative steps towards the final design could then proceed. However, as the rotor blade is to be constructed from composite materials, it would be necessary to deal with the mechanics of composite laminates. To this end, it is useful to first review some of the basic formulations and principles of composite material mechanics. This will be done in the next chapter. 


\section{CHAPTER 3 REVIEW OF COMPOSITE MATERIAL ANALYSIS}

\subsection{Introduction}

The SHARCS rotor blade is to be fabricated with composite materials. Due to the orthotropic behaviour of composite plies, analysis of such a composite structure must take into account the directional variations of the material properties. Standard equations for structural analysis that assume isotropic material properties would therefore not be appropriate for determining the stresses and strains. Thus, it is useful first to review classical laminate theory in the mechanics of composites. Only the key equations are presented in what follows as details of their development are well established in the literature, see, e.g. (Kaw 1997, Kollar and Springer 2003, Hoskin and Baker 1986).

\subsection{Classical Laminate Theory}

A composite laminate is constructed by stacking a number of uni-directional laminae (e.g. Figure 3.1), each lamina being a thin layer of fibre reinforced matrix, such as carbon 
fibre reinforced polymer. The classical laminate theory takes into account the directional nature of the mechanical properties of a uni-directional (UD) ply, which is orthotropic.
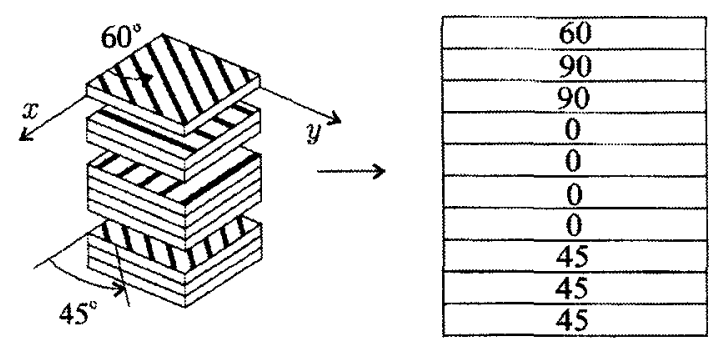

Figure 3.1: Example laminates [Kollar 2003]

Consider the three-dimensional stress-strain relationship for a generally anisotropic, linearly elastic material, given by Eq. (3.1). The direct stresses $\left(\sigma_{i}\right)$, direct strains $\left(\varepsilon_{i}\right)$, shear stresses $\left(\tau_{i j}\right)$ and shear strains $\left(\gamma_{i j}\right)$ vary directionally and they are related by the stiffness matrix elements $\left(C_{i j}\right)$. The elements of the stiffness matrix $([C])$ in the $x, y, z$ coordinate system depend on the properties for the individual uni-directional layers weighted over the layer thickness, see, e.g. (Kollar and Springer, 2003).

$$
\left(\begin{array}{l}
\sigma_{x} \\
\sigma_{y} \\
\sigma_{z} \\
\tau_{y z} \\
\tau_{z x} \\
\tau_{x y}
\end{array}\right)=\left(\begin{array}{cccccc}
C_{11} & C_{12} & C_{13} & C_{14} & C_{15} & C_{16} \\
& C_{22} & C_{23} & C_{24} & C_{25} & C_{26} \\
& & C_{33} & C_{34} & C_{35} & C_{36} \\
& & & C_{44} & C_{45} & C_{46} \\
& s y m . & & & C_{55} & C_{56} \\
& & & & & C_{66}
\end{array}\right)\left(\begin{array}{l}
\varepsilon_{x} \\
\varepsilon_{y} \\
\varepsilon_{z} \\
\gamma_{y z} \\
\gamma_{z x} \\
\gamma_{x y}
\end{array}\right)
$$


This equation can be subdivided into a more common form shown in Eq. (3.2), where the sub-matrix " $A$ " relates the direct stresses to direct strains; sub-matrix " $B$ " "couples" the direct stresses to shear strains; and sub-matrix " $D$ " relates the shear stresses to shear strains.

$$
\left(\begin{array}{l}
\sigma \\
\tau
\end{array}\right)=\left(\begin{array}{ll}
A & B \\
B & D
\end{array}\right) \cdot\left(\begin{array}{l}
\varepsilon \\
\gamma
\end{array}\right)
$$

\subsection{Classical Laminate Theory Simplifications}

The $A-B-D$ form of Eq. (3.1) as shown in Eq. (3.2) is so reduced to facilitate simplifications for numerical calculations. Due to the required symmetric NACA 0012 profile, any bending would produce equal magnitude stresses on the top and bottom surface of the airfoil, eliminating warping of the cross section if the lay-up is also symmetric. Since the airfoil cross-section cannot warp, the laminate cannot be deformed out of the plane of the cross-section. Therefore, an exclusive 2-D plane-stress state exists in the rotor blade cross-section. From that the out-of-plane shear stresses $\left(\tau_{x z}, \tau_{y z}\right)$ and out-of-plane direct stress $\left(\sigma_{z}\right)$ can be set to zero, for a thin lamina. This assumption remains valid for the rotor blade laminate analysis.

A further simplification can be made based on the symmetric cross section of the airfoil. The global stress calculations are done in local coordinates (Chapter 4), which are 
parallel to the symmetric axis of the airfoil. Thus the local coordinates are also the principal axis for the airfoil, resulting in the decoupling of shear stresses caused by bending strain and axial stresses. With that, the sub-matrix (" $B$ ") can be set to zero. Thus, the $6 \times 6$ stiffness matrix of Eq. (3.1) is reduced to a $3 \times 3$ matrix as given by Eq. (3.3).

$$
\left(\begin{array}{l}
\sigma_{x} \\
\sigma_{y} \\
\tau_{x y}
\end{array}\right)=\left(\begin{array}{ccc}
C_{11} & C_{12} & C_{16} \\
& C_{22} & C_{26} \\
\text { sym. } & & C_{66}
\end{array}\right) \cdot\left(\begin{array}{l}
\varepsilon_{x} \\
\varepsilon_{y} \\
\gamma_{x y}
\end{array}\right)
$$

Due to the orthotropy of uni-directional composite lamina, Eq. (3.3) can be further simplified. The local coordinates can be set to be along the principal axes of the rotor blade, so the fibre orientation is the same as this axis. Thus the fibre stress would be along the span axis and there would not be in-plane shear deformation. With this, the $C_{l 6}$ and $C_{26}$ terms can be reduced to zero. Thus Eq. (3.3) can be rewritten as follows.

$$
\left(\begin{array}{l}
\sigma_{1} \\
\sigma_{2} \\
\tau_{12}
\end{array}\right)=\left(\begin{array}{ccc}
C_{11} & C_{12} & 0 \\
& C_{22} & 0 \\
\text { sym. } & & C_{66}
\end{array}\right) \cdot\left(\begin{array}{c}
\varepsilon_{1} \\
\varepsilon_{2} \\
\gamma_{12}
\end{array}\right)
$$

where, the subscript " 1 " denotes parallel, " 2 " denotes in-plane perpendicular, and " 3 " refers to out-of-plane perpendicular to the fibre direction.

Figure 3.2 shows an example of the lamina co-ordinate system. 


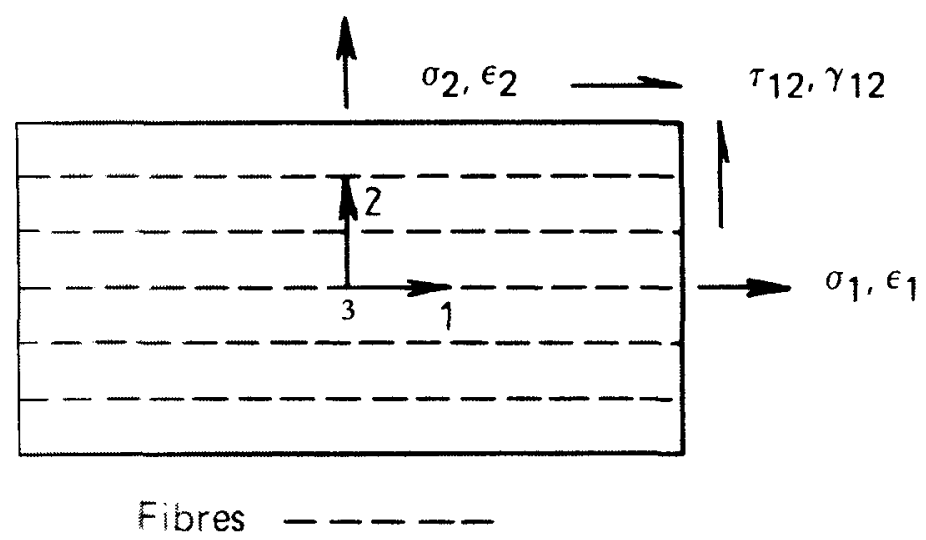

Figure 3.2: Lamina co-ordinate system

\subsection{Laminate Theory}

The stiffness coefficients $C_{i j}$ in Eqs. (3.1) - (3.4) are directly related to the more commonly used engineering constants, namely, Young's moduli, $E_{i}$, shear modulus $G_{i j}$, and Poisson's ratio, $v_{i j}$. In terms of these material constaints, Eq. (3.4) can be written as follows:

$$
\left(\begin{array}{l}
\sigma_{1} \\
\sigma_{2} \\
\tau_{12}
\end{array}\right)=\frac{1}{1-v_{12} \cdot v_{21}} \cdot\left(\begin{array}{ccc}
E_{1} & v_{12} \cdot E_{2} & 0 \\
v_{21} \cdot E_{1} & E_{2} & 0 \\
0 & 0 & \left(1-v_{12} \cdot v_{21}\right) \cdot G_{12}
\end{array}\right) \cdot\left(\begin{array}{c}
\varepsilon_{1} \\
\varepsilon_{2} \\
\gamma_{12}
\end{array}\right)
$$

As all the properties of the uni-directional layer required in Eq. (3.5) are in the parallel or perpendicular directions to the global axes, a co-ordinate transformation of Eq. (3.5) is required for these plies that do not have the fibres parallel with the global axes (Figure 
3.3). The steps for this transformation may be obtained from, e.g. Kollar and Springer (2003). The resulting 3 by 3 stiffness matrix in the global coordinate system $\left(\left[C_{G}\right]\right)$ obtained after the transformation will, in general, be fully populated.

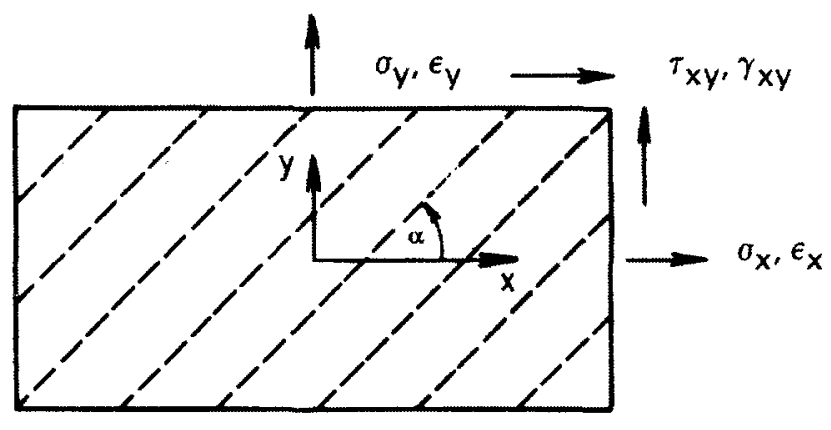

Fibres - - - -

Figure 3.3: Angle lamina

As each lamina in the laminate has a different stiffness matrix (denoted with subscript $i$ ), each must be transformed into the global coordinate system using the transformation matrix (Eq. 3.6).

$$
T=\left(\begin{array}{ccc}
\cos ^{2} \alpha & \sin ^{2} \alpha & 2 \cdot \sin \alpha \cdot \cos \alpha \\
\sin ^{2} \alpha & \cos ^{2} \alpha & -2 \cdot \sin \alpha \cdot \cos \alpha \\
-\sin \alpha \cdot \cos \alpha & \sin \alpha \cdot \cos \alpha & \cos ^{2} \alpha-\sin ^{2} \alpha
\end{array}\right)
$$

As the airfoil was symmetric any bending stiffness effects of the ply lay-up would cancel with that of the opposite side of the airfoil. Also, due to the large distance from the 
neutral axis, the ply lay-up order effects would be negligible. Therefore transformed matrix could then simply be superimposed to form a 3 by 3 total stiffness matrix $([\bar{C}])$ for the laminate, as follows:

$$
\bar{C}=\sum_{i=1}^{n}\left(\frac{t_{i}}{t} \cdot C_{G, i}\right)
$$

where $\quad n=$ total number of lamina in the laminate

$$
\begin{aligned}
& t=\text { total thickness of laminate } \\
& t_{i}=\text { thickness of lamina } \\
& C_{G, i}=\text { global stiffness matrix of lamina } i
\end{aligned}
$$

The effective engineering constants required for subsequent calculations can be obtained by inverting the laminate stiffness matrix to obtain the compliance matrix. It should be noted that only the superposition of the stiffness matrix, not the compliance matrix, results in the correct laminate properties.

$$
[\bar{S}]=\left(\begin{array}{ccc}
S_{11} & S_{12} & S_{16} \\
& S_{22} & S_{26} \\
s y m & & S_{66}
\end{array}\right)=[\bar{C}]^{-1}=\left(\begin{array}{ccc}
C_{11} & C_{12} & C_{16} \\
& C_{22} & C_{26} \\
s y m & & C_{66}
\end{array}\right)^{-1}
$$




$$
\begin{array}{lll}
E_{x}=\frac{1}{S_{11}} & (3.9) & v_{x y}=-\frac{S_{12}}{S_{11}} \\
E_{y}=\frac{1}{S_{22}} & (3.11) & v_{y x}=-\frac{S_{12}}{S_{22}} \\
G_{x y}=\frac{1}{S_{66}} & (3.13)
\end{array}
$$

With the effective engineering constants of the laminate, the global strains of the rotor blade can now be calculated. The lamina strains $\left[\varepsilon_{i}\right]$ were obtained by transforming the global strains $\left[\varepsilon_{G}\right]$ about the lamina angle $\alpha_{i}$,

$$
\left[\varepsilon_{i}\right]=[T] \cdot\left[\varepsilon_{G}\right]
$$

where: $[T]$ is the transformation matrix, given previously by Eq. (3.6)

With the lamina strains calculated, the lamina stresses can be obtained. This would be required for determining the safety factor, which will be calculated in Chapter 5. 


\subsection{Concluding Remarks}

The relationship between the stresses and strains in a composite laminate has now been briefly reviewed and how the effective engineering constants may be determined has also been explained. In order to establish the final dimensions as well as the appropriate layup of the composite that would be used for the rotor blade, the load conditions on the blade must be known. They include various aerodynamic, inertial and static loads. These will be discussed in the following chapter. 


\section{CHAPTER 4 ROTOR BLADE LOADS}

\subsection{Introduction}

The forces acting on the rotor blade must be determined in order to establish its final dimensions; they include the aerodynamic and inertial loads. A load calculation computer program developed at the German Aerospace Center (Deutsches Zentrum für Luft- und Raumfahrt [DLR]) (Wierach, 2004 and Moller 2004) was used for this purpose. The required input values for the program are shown in Table 4.1.

Table 4.1: Input data for the rotor blade load program

\begin{tabular}{lclc}
\hline \hline Mass $\left(m_{x}\right)[\mathrm{kg} / \mathrm{m}]$ & 0.5355 & Chord $(c)[\mathrm{m}]$ & 0.0753 \\
Rotational Speed $(\Omega)[\mathrm{rad} / \mathrm{s}]$ & 150 & Angle of Attack $(\alpha)\left[{ }^{\circ}\right]$ & 5 \\
Hub Radius $\left(r_{0}\right)[\mathrm{m}]$ & 0.126 & No. of blades $(z)$ & 4 \\
Outer Radius $(R)[\mathrm{m}]$ & 1.126 & $F_{\text {Maint }}[\mathrm{N}]$ & 20 \\
Air Density $\left(\rho_{\infty}\right)\left[\mathrm{kg} / \mathrm{m}^{3}\right]$ & 1.225 & & \\
\hline
\end{tabular}

The details for some of the information presented in this table have been explained in Chapter 2. The program can calculate the various loads at 100 discrete locations along the blade span. As the twist profile specified was not linear, the program was modified 
for the present work, wherein the twist values at the discrete radius locations were obtained by interpolation. Any mass variations due to actuation systems were manually added to the specific rotor span position within the program. In the following sections, the relevant formulae used in this program for the calculation of the various loads are explained and the results of the computations are presented.

\subsection{Centrifugal Load}

Due to the high rotational speed of $150 \mathrm{rad} / \mathrm{s}(1433 \mathrm{rpm})$ the most significant load in the design of the rotor blade is the centrifugal force. The elemental centrifugal force, $d F_{C F}$, acting on an infinitesimal blade element may be written as Eq. (4.1).

$$
d F_{C F}=d m \cdot \Omega^{2} \cdot r
$$

Where $d m$ is the mass of the blade element, and $\Omega$ is the rotational speed. An important point to note is that the moment due to the centrifugal force about the flapping hinge balances the bending caused by the aerodynamic forces, since the moment about this hinge is zero. Thus, the centrifugal load helps to ensure that the blade remains almost perpendicular to the rotational axis. This effect is illustrated in Figure 4.1. 


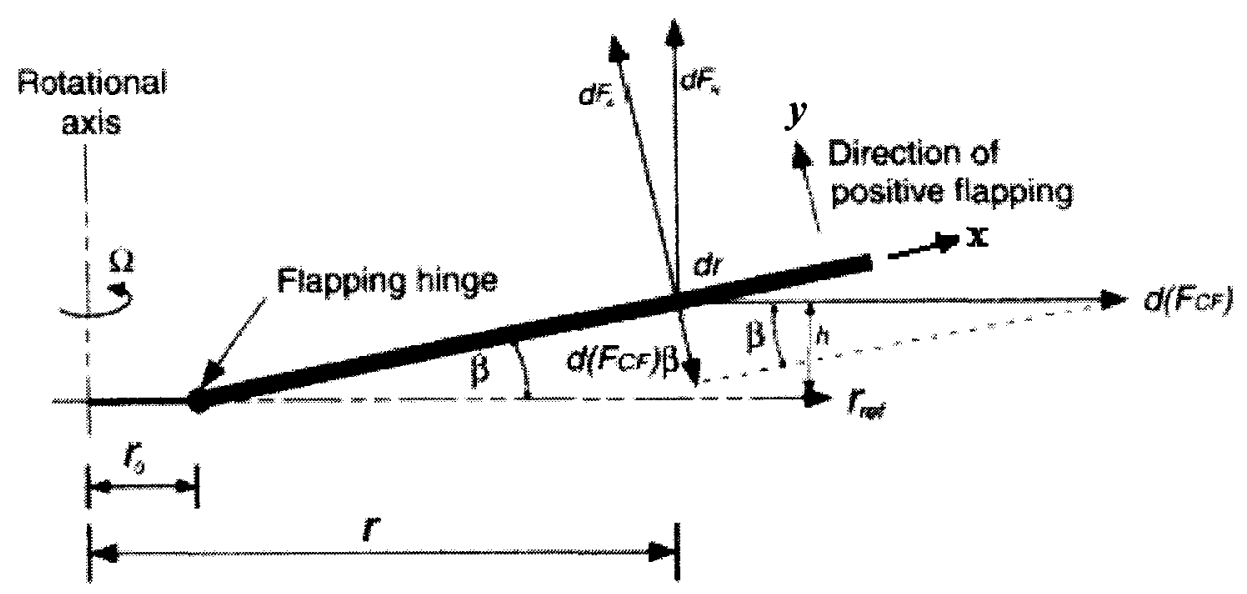

Figure 4.1: Force equilibrium about the rotor blade flapping hinge [Leishman 2000]

Equation (4.1) clearly indicates that as $r$ increases so does the centrifugal force on the blade section. The centrifugal force at a given radial location ( $r$ ) to the tip of the blade $(R)$ can be obtained as follows.

$$
F_{C F}=\int_{r}^{R} d F_{C F} \cdot d r
$$

It is evident that the maximum centrifugal force experienced by the blade occurs at the root. Figure 4.2 shows that for the SHARCS rotor blade, the maximum centrifugal load is $7500 \mathrm{~N}$. The discrete slope changes seen in Figure 4.2 are due to the discrete masses of the actuation systems. In particular, the approximate $1000 \mathrm{~N}$ increase at $r=1.03 \mathrm{~m}$ was due to the estimated $30 \mathrm{~g}$ for the anhedral tip hinge. The load increase was relatively large because additional mass was placed a large distance from the axis of rotation. At this early stage of the design process only an estimate of $30 \mathrm{~g}$ for the anhedral tip hinge and $15 \mathrm{~g}$ for the flap actuator were available. 


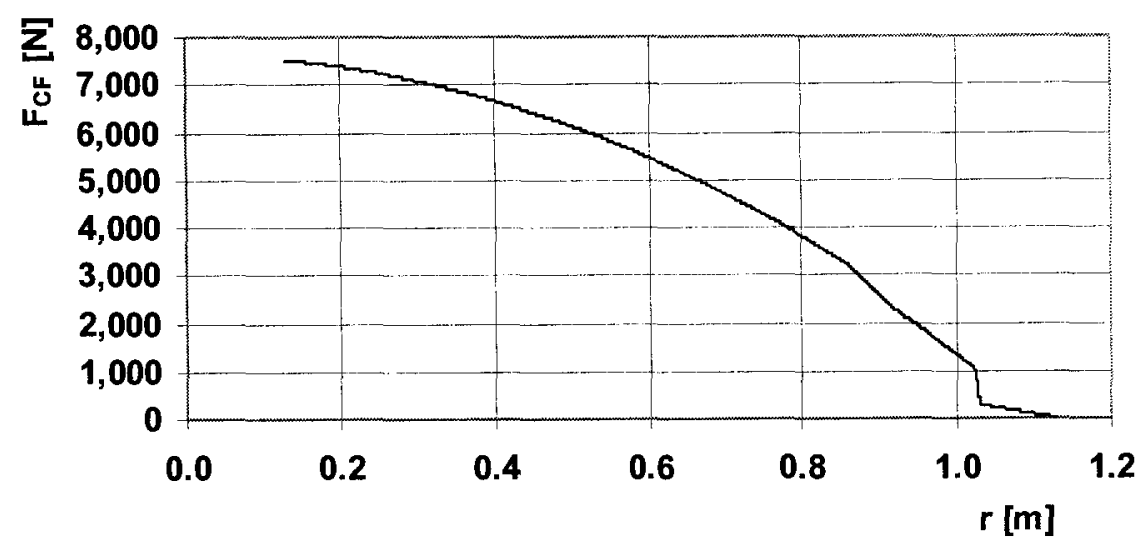

Figure 4.2: Centrifugal force along the span of the rotor blade

\subsection{Angle of Attack}

To determine the aerodynamic loads on the blade, the angle of attack distribution was required. A rotor blade creates an induced downward air velocity $\left(v_{i}\right)$. This induced airflow modifies the pitch of the blade $(\theta)$ to the effective angle of attack $(\alpha)$ by the induced angle $(\phi)$, as shown in Figure 4.3. The effect of the induced airflow would be more pronounced at the blade tip than the blade root. As this effect also occurs for fixed wing aircraft, the same methods used to minimize it could be applied. Fixed wings employ a twist distribution to negate the induced velocity effect. For the SHARCS rotor blade the twist profile was set in the design requirements and is defined by the $\theta$ curve on Figure 4.4. 


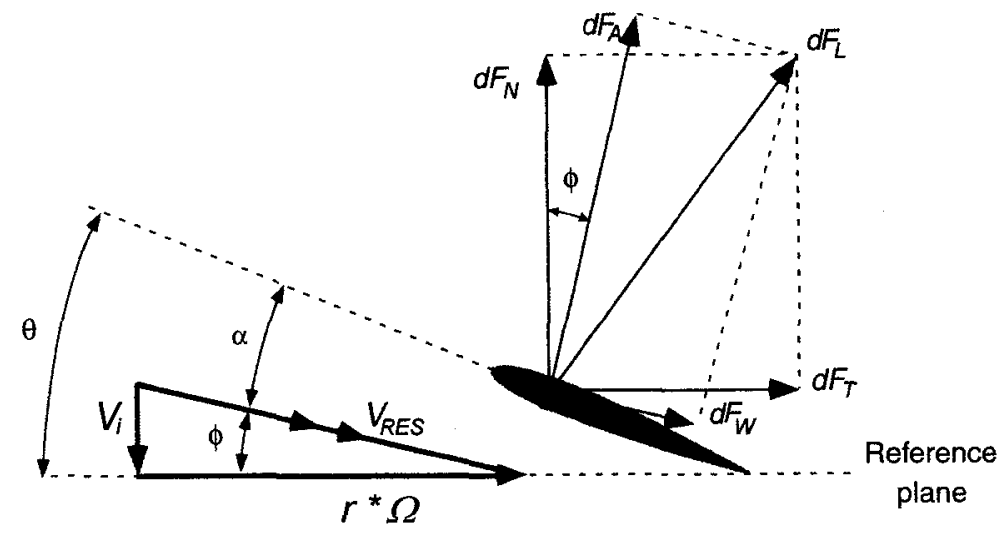

Figure 4.3: Incident velocities and aerodynamic forces for a rotor blade element

[Leishman 2000]

As there was a defined twist profile, the induced airflow would change along the span.

From Blade Element Momentum Theory (BEMT), the induced airflow equation is given by Leishman (2000):

$$
v_{i}(r)=\frac{z \cdot \frac{d C_{L}}{d \alpha} \cdot c \cdot \Omega}{16 \cdot \pi} \cdot\left[\sqrt{1+\frac{32 \cdot \pi \cdot r \cdot \theta}{z \cdot \frac{d C_{L}}{d \alpha} \cdot c}}-1\right]
$$

where: $z=$ number of blades $=4$

$$
\begin{aligned}
& c=\text { chord length }=0.0753 \mathrm{~m} \\
& \frac{d C_{L}}{d \alpha}=\text { lift curve slope; assumed: } 2 \pi
\end{aligned}
$$


The lift curve slope, $\mathrm{C}_{\mathrm{L} \alpha}$ is the derivative of the coefficient of lift with respect to the angle of attack for the airfoil, and can be visualized as the slope of the coefficient of lift curve when plotted against the angle of attack (Appendix B). As the coefficient of lift

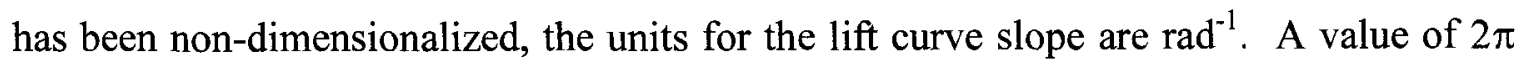
was used for the lift curve slope, as that would be the maximum theoretical lift curve slope. As such, it would lead to the highest aerodynamic forces, thereby yielding a structurally conservative design. To calculate the induced angle $(\phi)$ from the induced velocity, a small angle assumption was made to obtain the following equation [Leishman 2000]:

$$
\tan (\phi(r)) \cong \phi(r)=\frac{v_{i}(r)}{\Omega \cdot r}
$$

Following which the effective angle of attack $(\alpha)$ can be determined as:

$$
\alpha(r)=\theta(r)-\phi(r)
$$

Combining Eq. (4.3-4.5) with the required twist profile and a root pitch angle of $5^{\circ}$, the angle of attack profile shown in Figure 4.4 was obtained. Conservatively a root angle of attack of $5^{\circ}$ was chosen to determine the ultimate aerodynamic forces the blade would experience. In fact, Figure 4.4 shows that while the pitch of the rotor blade has a maximum of $9^{\circ}$, the angle of attack only reached $3^{\circ}$ due to the inflow velocity. 


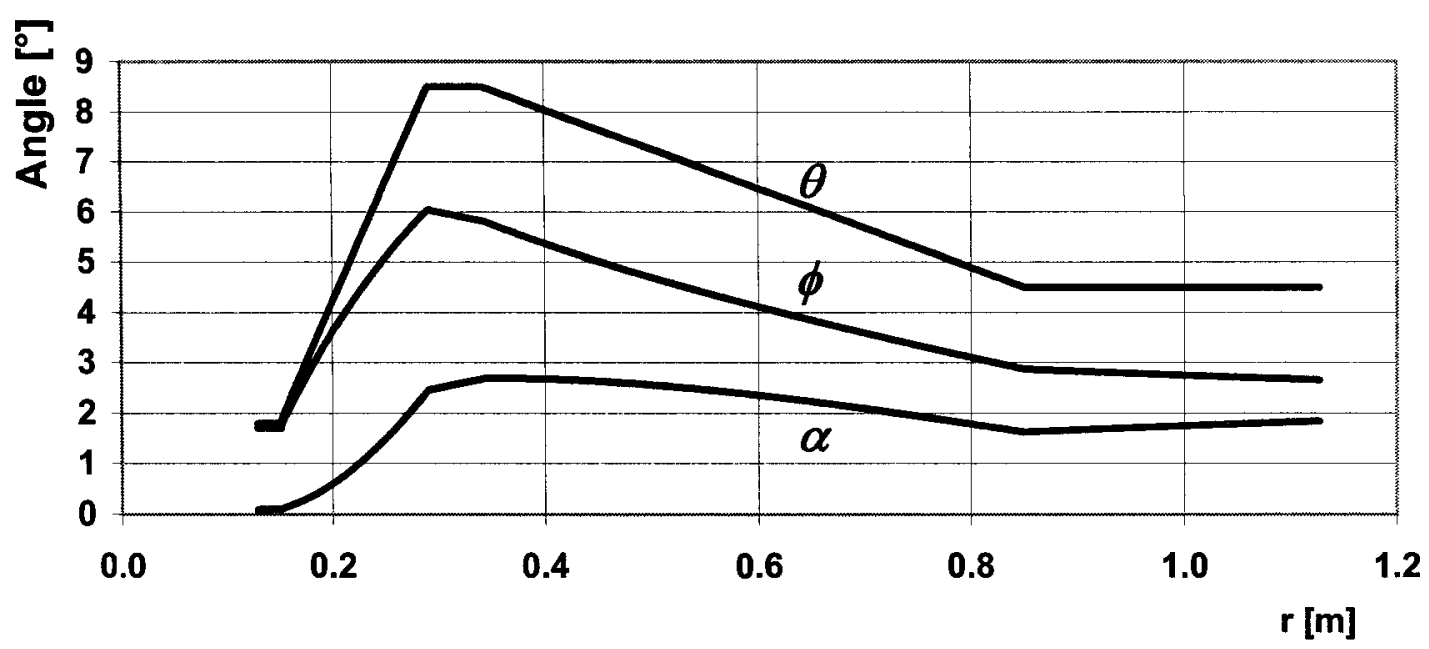

Figure 4.4: Variations of pitch $(\theta)$, induced $(\phi)$ and angle of attack $(\alpha)$ along the span of the rotor blade

\subsection{Lift}

The primary purpose of a rotor blade is the creation of lift. The same basic equation for the determination of lift for a fixed wing aircraft applies to rotorcraft with a difference, however, in that for the velocity of the blade varies linearly along the span due to the rotational velocity $\Omega$. Thus, the elemental force due to lift $\left(d F_{L}\right)$ may be expressed as:

$$
d F_{L}=\frac{1}{2} \cdot \rho_{\infty} \cdot(\Omega \cdot r)^{2} \cdot \alpha \cdot \frac{d C_{L}}{d \alpha} \cdot c \cdot d r
$$

Substitution of the equations for the angle of attack, Eq (4.3 - 4.5), into Eq (4.6) results in the following expression for lift. 
$d F_{L}=\frac{1}{2} \cdot \rho_{\infty} \cdot(\Omega \cdot r)^{2} \cdot \frac{d C_{L}}{d \alpha} \cdot\left[\theta-\frac{z \cdot \frac{d C_{L}}{d \alpha} \cdot c}{16 \cdot \pi \cdot r} \cdot\left(\sqrt{1+\frac{32 \cdot \pi \cdot r \cdot \theta}{z \cdot \frac{d C_{L}}{d \alpha} \cdot c}}-1\right)\right] \cdot c \cdot d r(4.7)$

Using Eq. (4.7) the theoretical lift distribution for the rotor blade was calculated. Without the twist distribution, the theoretical lift distribution takes the form of a parabolic curve, with the magnitude increasing monotonically from the root to the tip of the blade.

For the SHARCS blade, the theoretical lift distribution deviates slightly from a parabola; the lift does not increase monotonically to the tip of the blade due to the leakage of highpressure air to the low-pressure surface at the tip. The actual lift reaches a peak at around $80-90 \%$ span then decreases to zero at the tip. This effect was taken into account by only using the theoretical lift distribution up to a certain span location; a procedure known as Prandtl's tip loss theory. The excess lift calculated by the theoretical lift distribution is cancelled out by the absence of lift at the blade tip. That relative span position, $B$, was calculated using the Gessow and Myers equation (Leishman 2000).

$$
B=1-\frac{c}{2 \cdot R}=1-\frac{0.0753}{2 \cdot 1.126}=0.967
$$

The effective rotor radius, $R_{\text {eff }}$, used to calculate the differential lift was then obtained using:

$$
R_{\text {eff }}=B \cdot R=0.967 \cdot 1.126=1.088 \mathrm{~m}
$$


With these parameters calculated, the lift profile for the SHARCS rotor blade was produced as shown in Figure 4.5. It shows that the twist profile increased the lift generated along the blade radius from 0.25 to $0.85 \mathrm{~m}$.

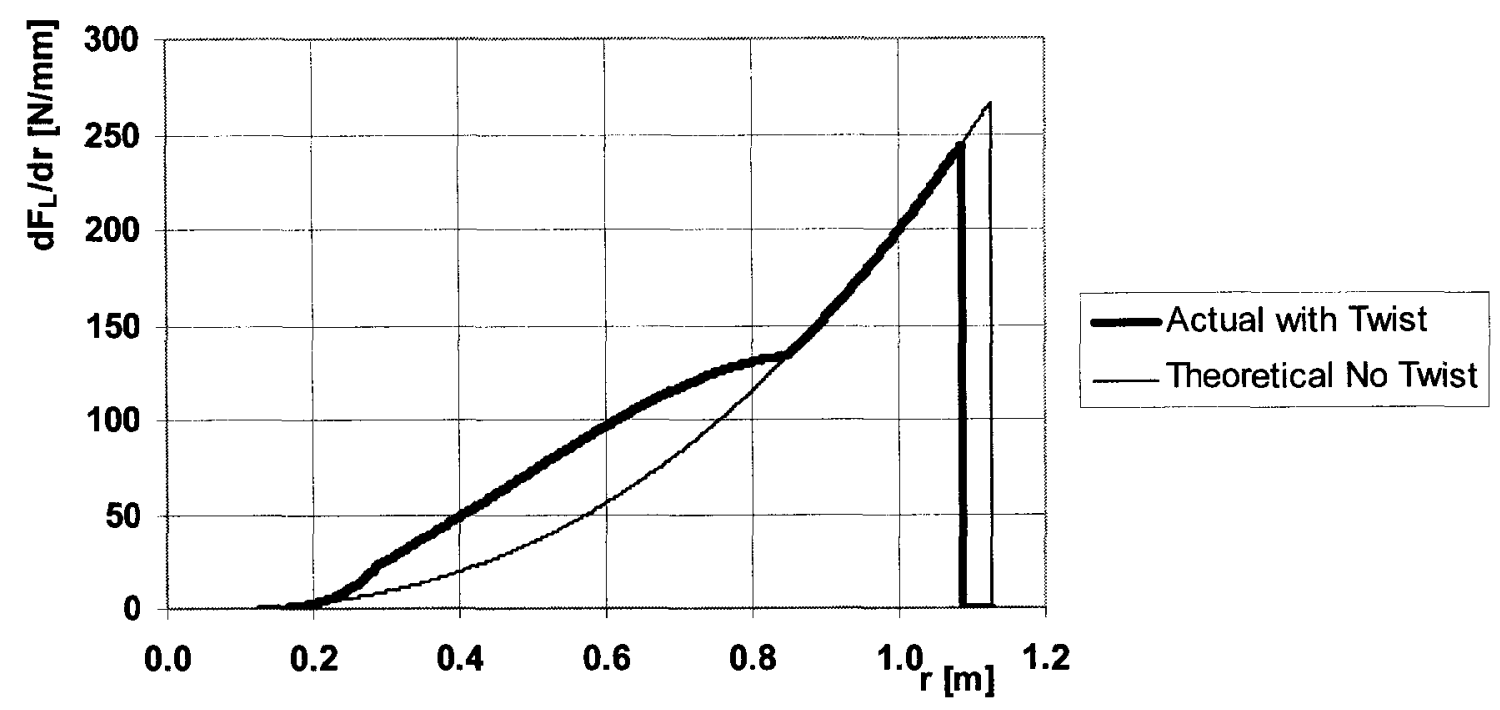

Figure 4.5: SHARCS actual and theoretical differential lift distribution along the span of the rotor blade

From the derivation of the lift equation, the differential lift, $d F_{L}$, acts perpendicular to the resultant velocity $\left(V_{\text {res }}\right)$, which means the normal of the rotor blade chord is effectively rotated by $\alpha$. As the rotor blade contains a twist, the lift does not act in the same direction along the span of the blade. Since, the maximum value for $\alpha$ was approximately $3^{\circ}$, and the twist angle has a range of $-3^{\circ}$ to $+4^{\circ}$, the following small angle approximation was made, $\cos (\alpha+$ twist $) \approx 1$. Using that approximation, the reaction force was calculated from: 


$$
F_{L}(r)=\int_{r}^{R_{e f f}} d F_{L}
$$

Figure 4.6 shows the reaction force distribution due to lift along the rotor blade, with the maximum of $93 \mathrm{~N}$ occurring at the blade root.

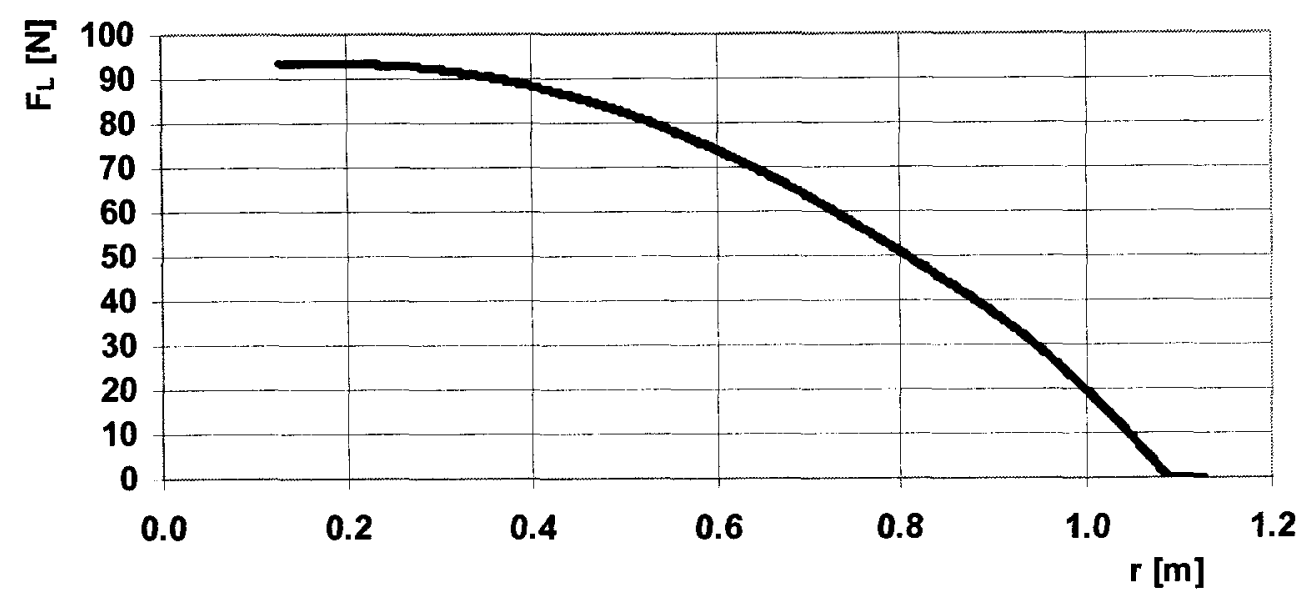

Figure 4.6: Variation of lift reaction force along the span of the rotor blade

\subsection{Drag}

The force equilibrium in the drag plane of the rotor blade is illustrated in Figure 4.7. A lead-lag hinge provides a pivot point, which causes a lead-lag angle $\zeta$. This angle is required to balance the Coriolis forces that appear when the blade flaps up and down. This motion changes the distance to the centre of mass of the rotor blade. With the centre of mass moving closer to the axis of rotation, the speed of rotation increases. 


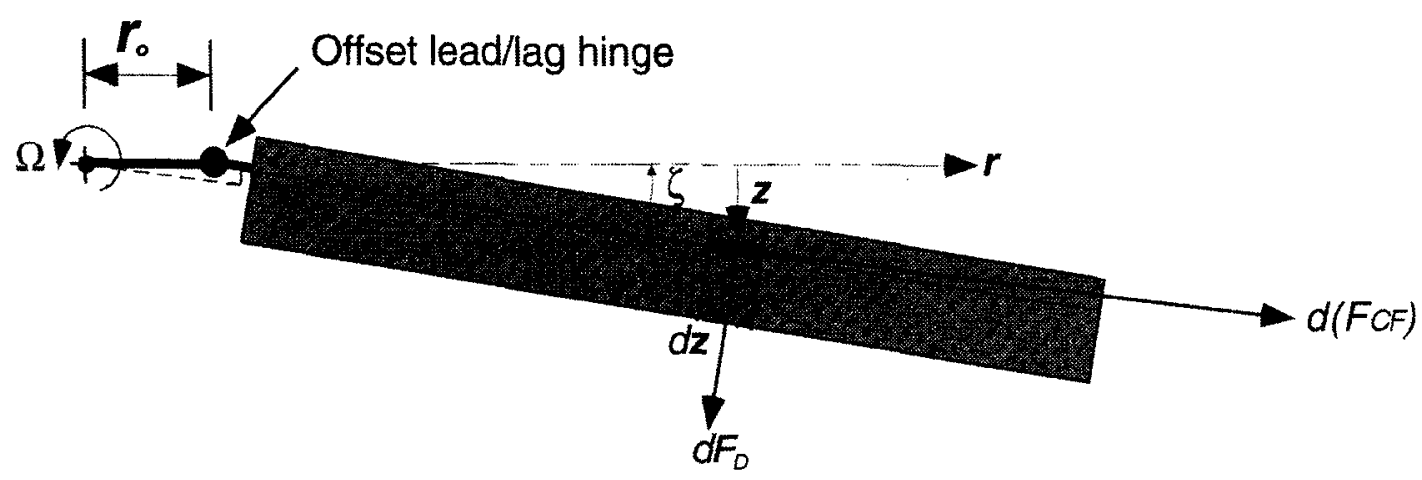

Figure 4.7: Force equilibrium about the lead-lag hinge [Leishman 2000]

The drag distribution was calculated in a similar manner to the lift distribution. Using the following equation, the drag was calculated along the blade radius.

$$
d F_{D}=\frac{1}{2} \cdot \rho \cdot(\Omega \cdot r)^{2} \cdot c \cdot C_{D} \cdot d r
$$

The drag coefficient $\left(C_{D}\right)$ consists of two parts, the parasitic $\left(C_{D o}\right)$ and induced $\left(C_{D 2}\right)$ drag coefficients. The following coefficients for the NACA 0012 profile were obtained from the drag polar and lift curves provided in Appendix B \& C [Abbott and Doenhoff 1959]:

$$
\begin{aligned}
& C_{D o}=0.01 \\
& C_{D 2}=0.4
\end{aligned}
$$

These coefficients could then be used to calculate the drag coefficient by rearranging Eq. (4.12a) [Raymer 1999] into Eq. (4.12b): 


$$
\begin{aligned}
& C_{D}=C_{D o}+k \cdot C_{L}{ }^{2} \\
& C_{D}=C_{D o}+C_{D 2} \cdot \alpha^{2}
\end{aligned}
$$

Substituting Eq. (4.12b) into (4.11), the drag distribution as shown in Figure 4.8 was obtained. It should be noted that the Prandtl tip loss theory was not used in the calculation of drag. This was because drag production was not reduced due to tip loss. The drag calculated near the blade tip assumed the tip generated the ideal amount of lift; therefore the result would be higher than in actual fact, resulting again in a conservative estimate.

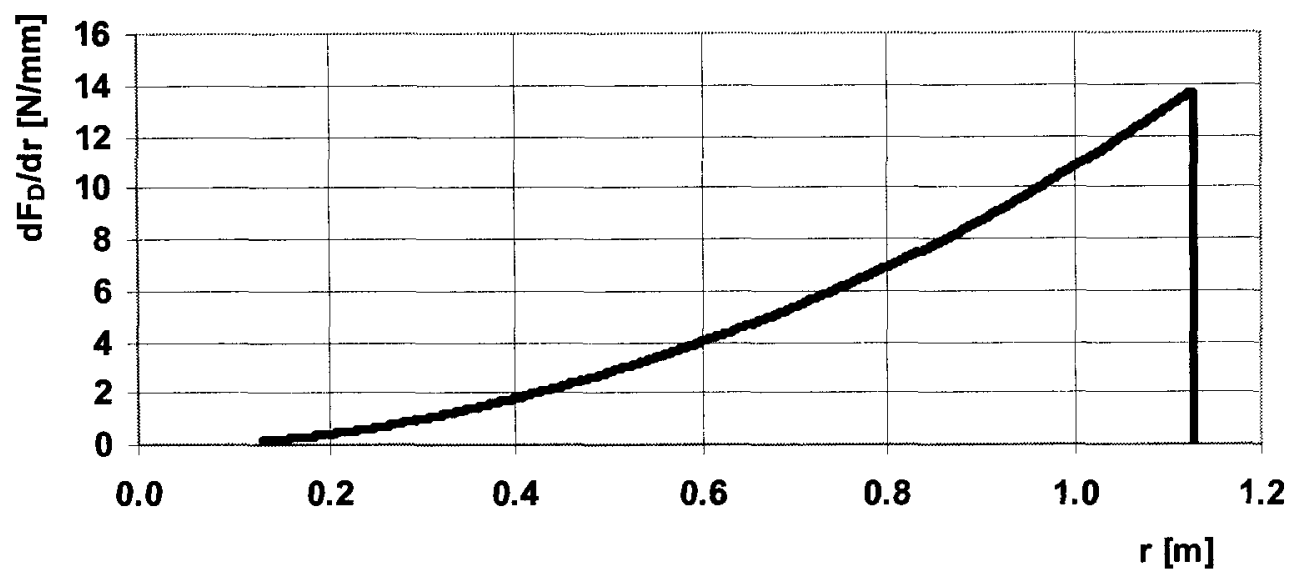

Figure 4.8: Differential drag force along the span of the rotor blade

As was considered for the lift calculations, small angles were assumed and the drag distribution was integrated to produce Figure 4.9. The maximum reaction force due to 
drag was found to be $5 \mathrm{~N}$ at the blade root, which was an order of magnitude smaller than the lift load, and two orders of magnitude smaller than the centrifugal load.

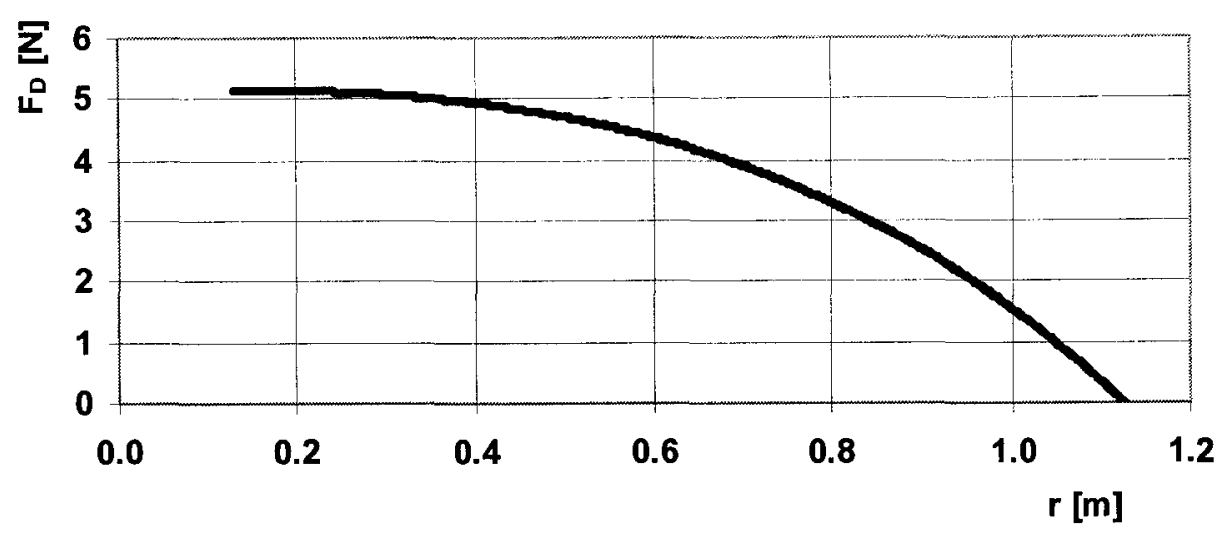

Figure 4.9: Drag reaction force along the span of the rotor blade

\subsection{Flapping and Lead-Lag Bending Moment}

As the rotor blade is articulated, no moment is transmitted to the rotor hub due to the flapping hinge. Therefore, the rotor blade moves freely up and down (flaps) depending on the amount of lift on the blade. The blade reaches equilibrium between the lift and centrifugal forces at a flapping angle, $\beta$. The previously calculated aerodynamic and inertial forces also produce a flapping and lead-lag bending moment along the blade. This is illustrated in Figure 4.10. 
The main cause of the bending moment is that the lift varies parabolically over the span of the blade, while the compensating centrifugal force only varies linearly. This happens in the general case, ignoring discrete mass changes and variations in the angle of attack due to twist. However, a resulting bending moment still would be present. The flapping and lead-lag moments must be calculated in order for the coning $(\beta)$ and lead-lag $(\zeta)$ angles to be determined.

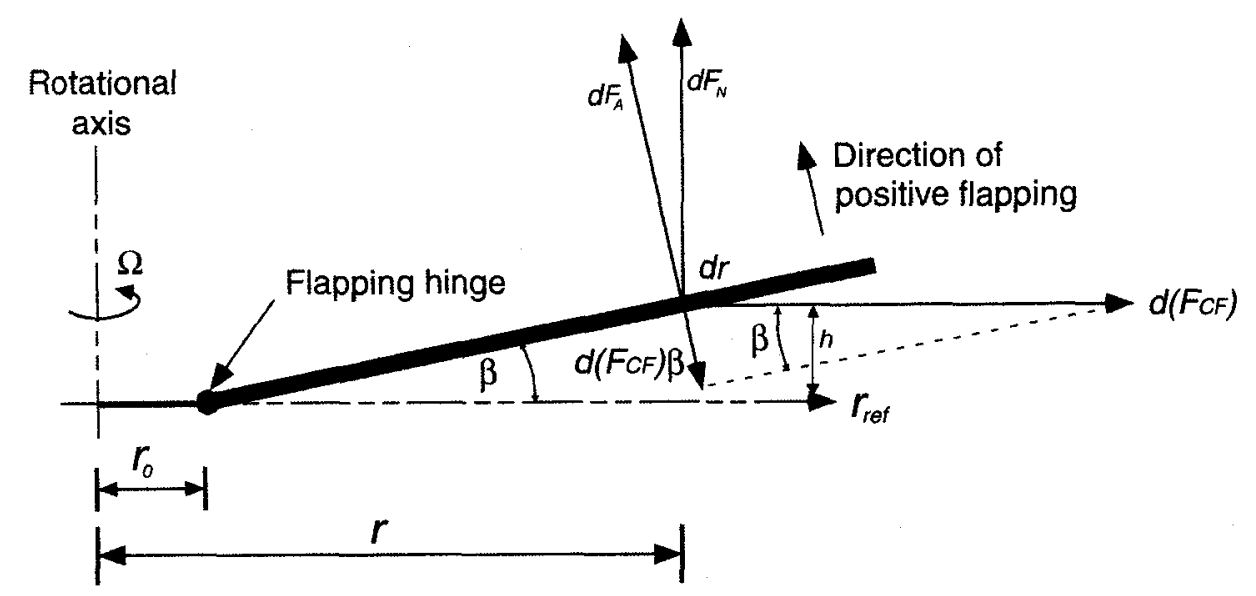

Figure 4.10: Force diagram for the flapping moment balance of a rotor blade

[Leishman 2000]

Assuming small angles $(\sin (\beta) \approx \beta, \cos (\beta) \approx 1)$, the following moment equation was derived from the free-body diagram shown in Figure 4.10.

$$
M_{\text {Flapping }}(r)=\int^{R}(R-r) \cdot\left(\frac{d F_{L}}{d r}-\sin (\beta) \cdot \frac{d F_{C F}}{d r}\right) \cdot d r
$$


Setting the moment equal to zero at the hinge location $\left(r=r_{o}\right)$, Eq. (4.1.3) can be rearranged into Eq. (4.14).

$$
\beta=\int_{r=r_{o}}^{R} \frac{d F_{L}}{d F_{C F}} \cdot d r
$$

An iterative process was then carried out until a value of $\beta$ was found that produced a zero moment at the hinge location. A value of $\beta=0.763^{\circ}$ was obtained. With the value for the coning angle, $\beta$, calculated, the bending moment distribution was then determined using Eq. (4.13). The reaction moment was obtained by integration, from the reaction force location to the blade tip. Figure 4.11 shows the variation of the reaction flapping moment along the span of the blade.

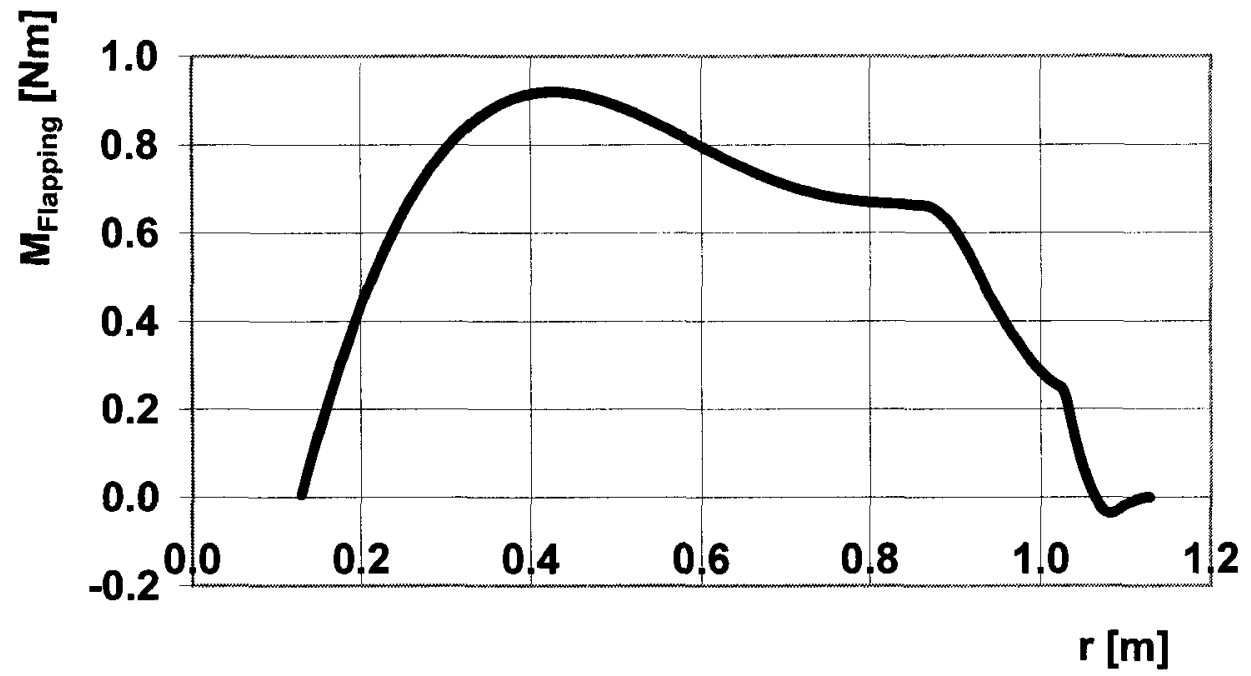

Figure 4.11: Distribution of the flapping moment along the span of the rotor blade 
The abrupt slope changes were caused by two different factors. One was the discrete mass changes caused by the vibration and noise reduction systems. The other was the Prandtl tip loss factor near the tip region. There, a discrete slope change in the bending moment occurs, exactly where the tip loss factor causes the lift to become zero. It is evident from Figure 4.10 that reducing the lift to zero a sudden change in the moment exerted on the blade at that location results. With no tip lift, the calculated moment would be slightly different from that experienced in practice.

The moment caused by drag (Figure 4.12) was substantially lower, due to the smaller magnitudes of the drag forces. Therefore, a maximum lead-lag moment of less than 0.2 $\mathrm{Nm}$, as opposed to the maximum flapping moment of $0.92 \mathrm{Nm}$ was obtained. The leadlag moment had smaller changes in slope as the lead-lag angle, $\zeta$, was an order of magnitude smaller then the coning angle, thereby reducing the influence of the centrifugal force. Also, the Prandtl tip factor was not used for drag, so there was no sudden change of the force to zero at the tip. 


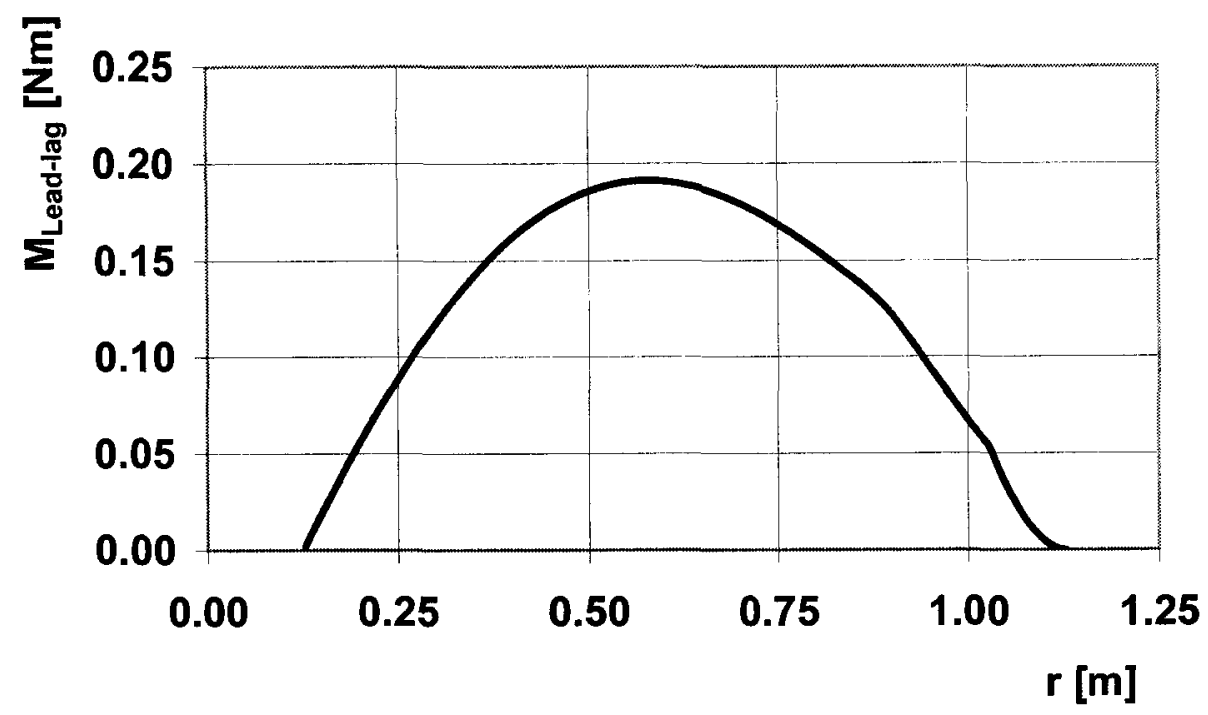

Figure 4.12: Lead-lag moment along the span of the rotor blade

\subsection{Miscellaneous Loads}

In addition to the described loads, one may consider in the design of the SHARCS blade other static loads, such as the ones from its self-weight. When the blade is not in operation, the weight of the blade needs to be supported. To prevent the blade from touching the ground at start up and shut down, the flapping hinge needs a stop. It that situation, the blade boundary conditions would no longer be pinned, but fixed at the root.

In addition to the static weight of the blade, a maintenance load may be applied to the blade. Due to the actuated systems integrated into the blade, it was deemed plausible that a mechanical force would be applied to the blade to adjust any of the systems, or to install 
the blade onto the hub. Therefore an arbitrary $20 \mathrm{~N}$ force was considered applied to the blade tip. This location was chosen, as it would produce the largest bending moment. As this load corresponds to a concentrated mass of about 4 times the blade mass applied at this location, this represents the maximum maintenance load that the blade could be expected to experience and withstand. The miscellaneous rotor loads are illustrated in Figure 4.13.

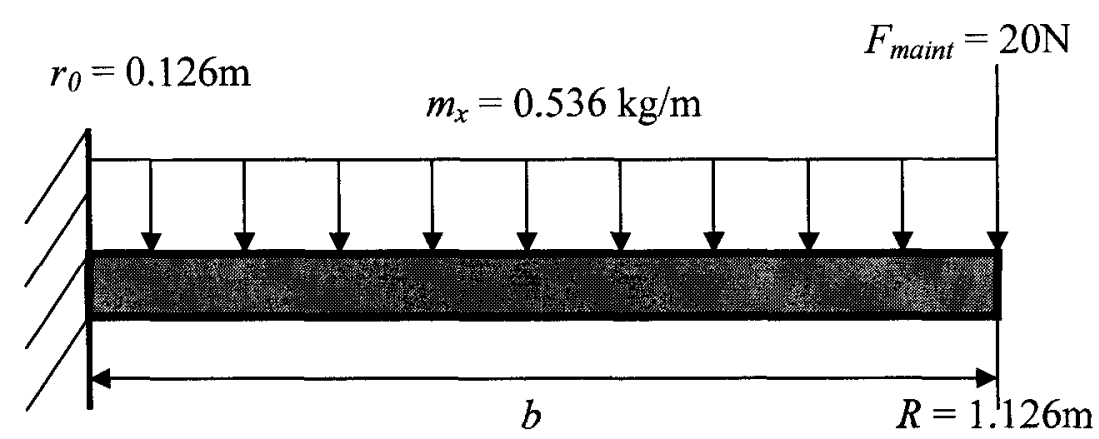

Figure 4.13: Rotor blade static forces

The shear force and bending moment along the span of the blade due to these static loads are thus given by Eq. (4.15) and Eq. (4.16), where $b$ is the total span of the blade.

$$
\begin{aligned}
& F_{\text {stat }}(r)=-F_{\text {maint }}-\frac{g \cdot \int_{r}^{R} d m}{b} \cdot(R-r) \\
& M_{\text {stat }}(r)=\left[(r-R) \cdot\left(\frac{g \cdot \int_{r}^{R} d m}{2 \cdot b}\right)-F_{\text {maint }}\right] \cdot(R-r)
\end{aligned}
$$


The discrete masses of the actuation system were considered in the analysis. The shear force and bending moment distributions are shown in Figures 4.14 and 4.15, respectively. Both the static force and the static moment were found to be significant when compared to the magnitudes of the other calculated loads. The maximum moment of $-22.5 \mathrm{Nm}$ was significantly larger than the maximum flapping moment of $0.92 \mathrm{Nm}$, indicating that blade mishandling may be a critical factor in the design.

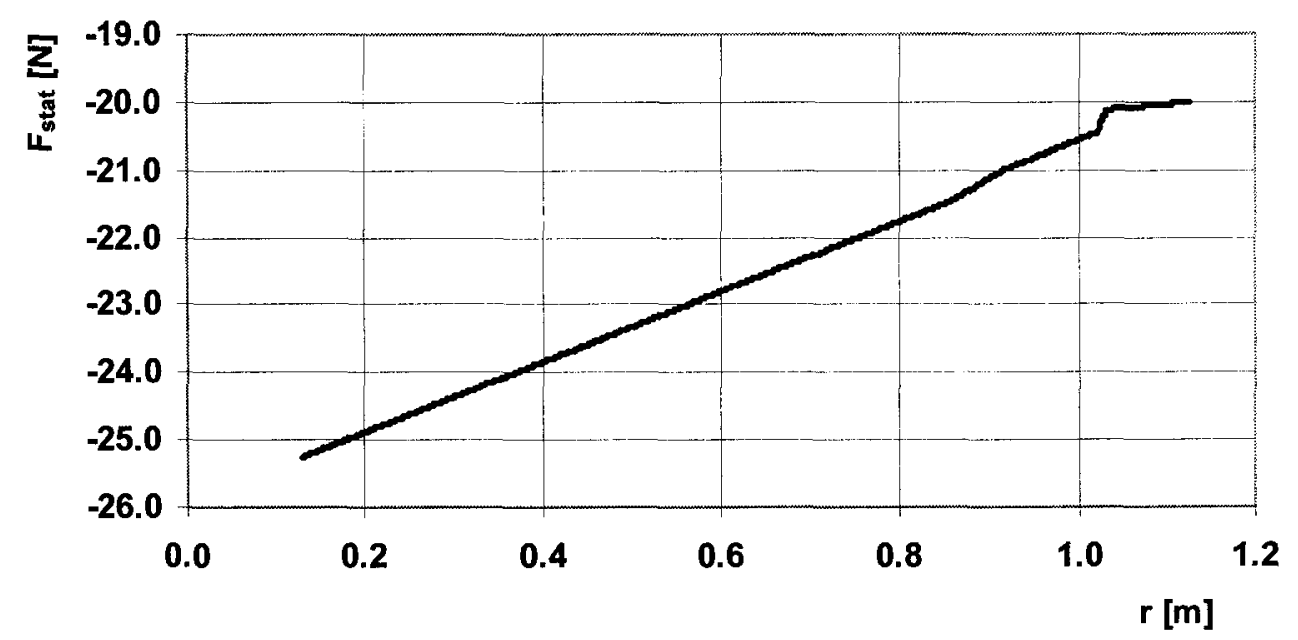

Figure 4.14: Resultant shear force along the span of the blade due to static loads 


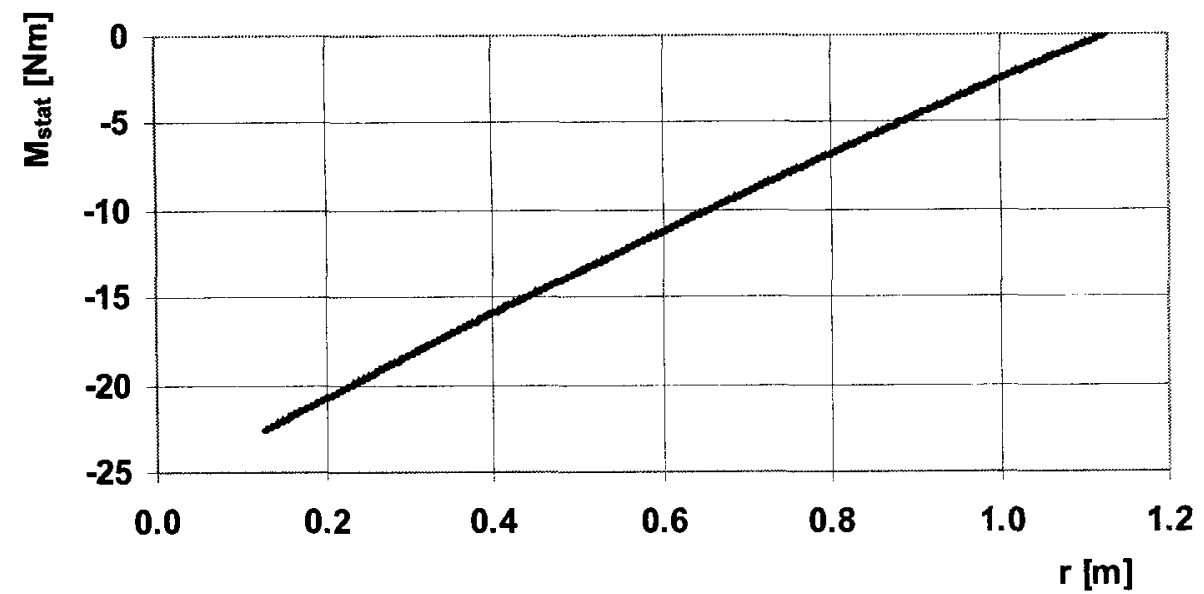

Figure 4.15: Bending moment distribution along the blade due to static loads

\subsection{X-Direction Force}

The calculated loads as discussed in the previous sections were transformed into the coordinate system chosen for the rotor blade (Figure 4.1 and 4.7) for the calculation of the laminate stresses and safety factors. As the x-direction was taken along the axis as the blade span, the force in that direction was completely comprised by the resultant centrifugal load. Therefore the force along the x-direction, $Q_{x}$, as shown in Figure 4.2, is identical to the resultant centrifugal load distribution shown in Figure 4.16, assuming $\beta$ to be small $(\cos \beta \cong 0)$. 


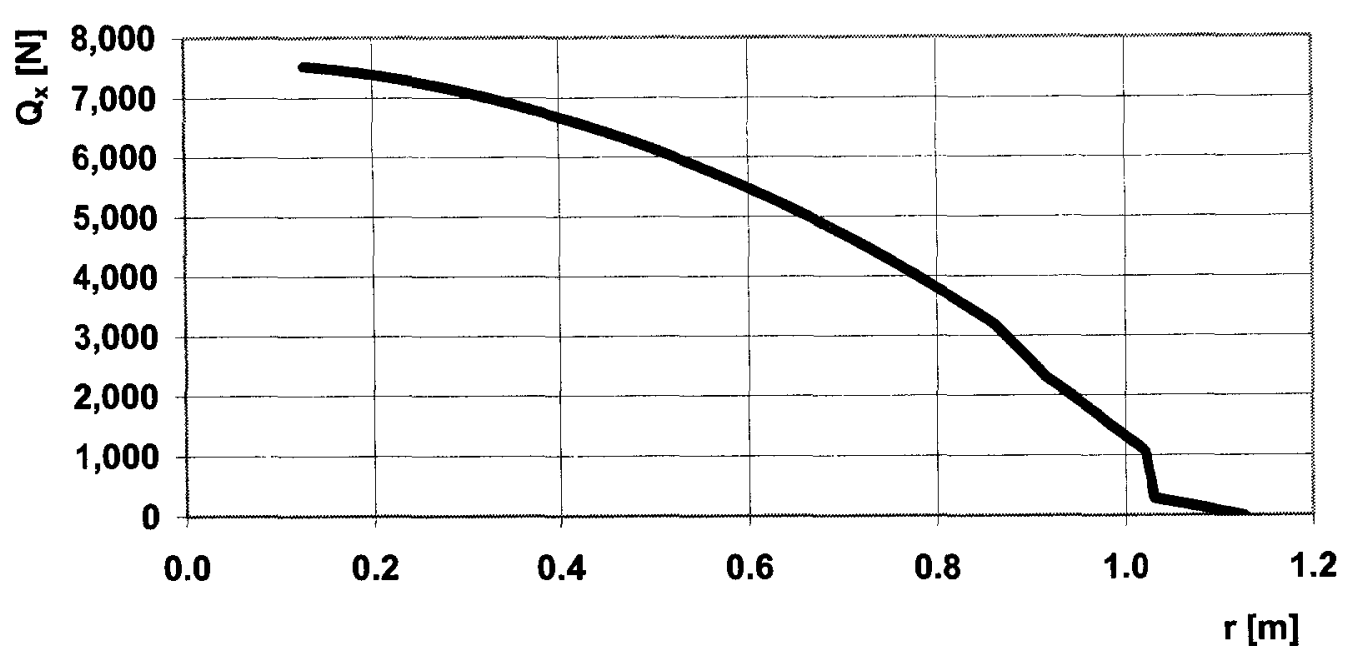

Figure 4.16: Variation of the force $Q_{x}$ along the span wise direction of the blade

\subsection{Y-Direction Force}

Shown previously in Figure 4.10, the y-direction force due to blade operation, $Q_{y}$, is comprised of the lift load and the resolved vertical component of the centrifugal load. The small angle assumption was not made here because the difference in magnitudes of the lift and centrifugal load caused the resolved vertical component of the centrifugal load to be significant. The resulting transverse shear force, $Q_{y}$, was then obtained from Eq. (4.17), and its distribution is illustrated in Figure 4.17.

$$
Q_{y}=F_{L}-\sin \beta \cdot F_{C F}
$$




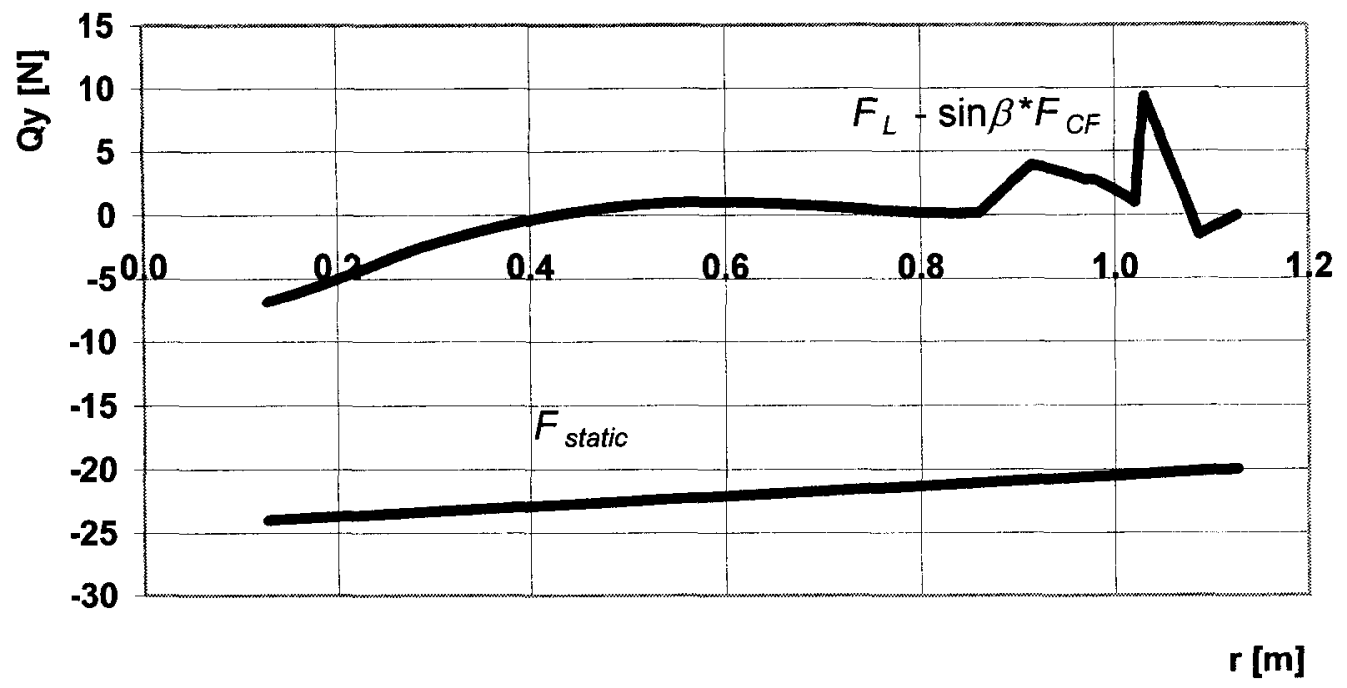

Figure 4.17: Static and dynamic transverse shear force along the span of the rotor blade

The static force was superimposed onto the previously described force to produce the maximum y-direction force distribution, shown in Figure 4.18. It should be noted that the maximum force was entirely comprised by the static load. 


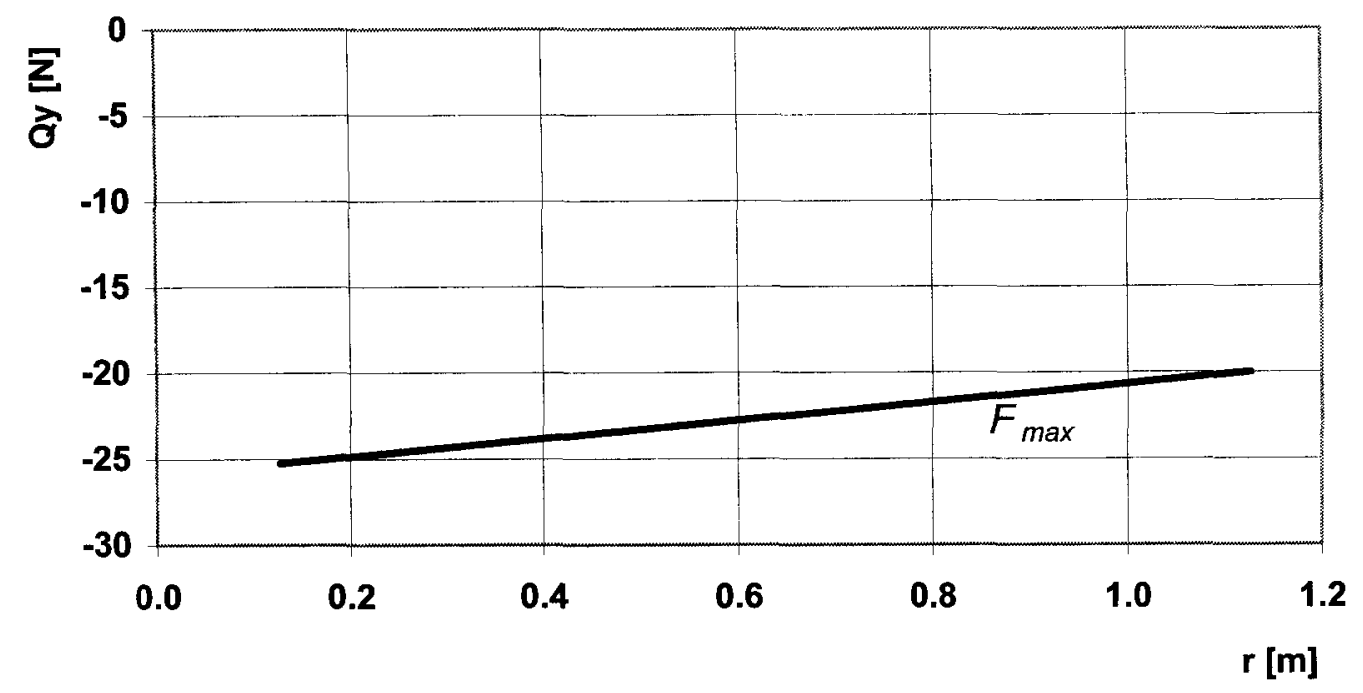

Figure 4.18: Maximum transverse shear force along the span of the rotor blade

\subsection{Z-Direction Force}

Similarly as described in the previous section, the z-direction shear force for the operational blade was comprised by the $\sin \zeta$ portion of the centrifugal load and the drag force. Figure 4.19 illustrates the $\mathrm{z}$-direction shear force distribution, with the maximum value being $1 \mathrm{~N}$.

$$
Q_{Z}=F_{D}-\sin \zeta \cdot F_{C F}
$$




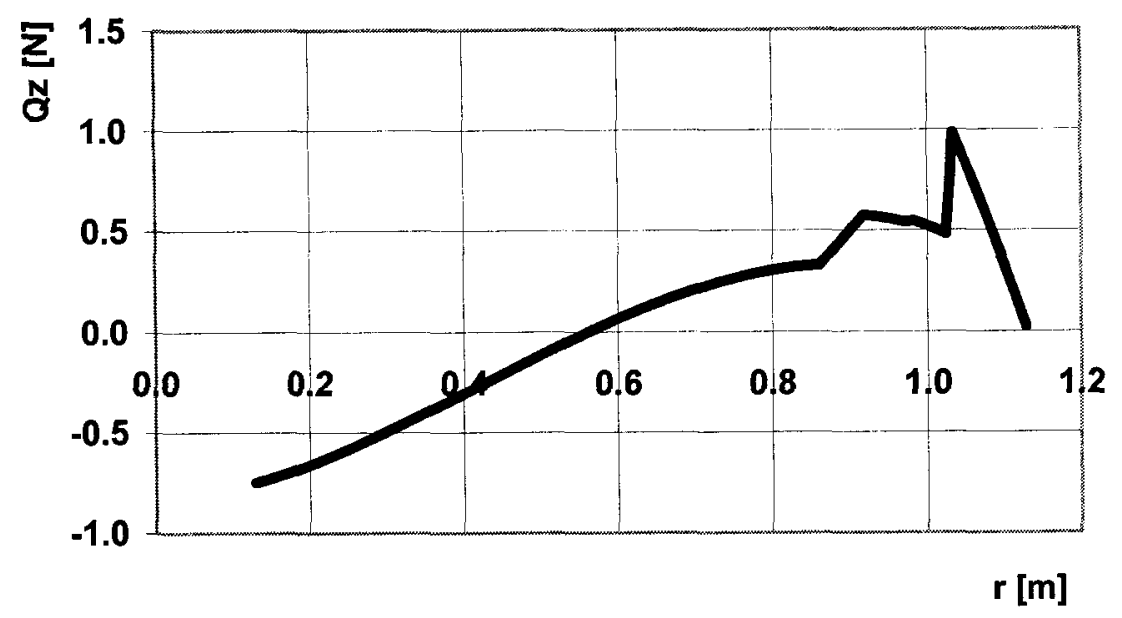

Figure 4.19: Z-axis shear force distribution along the span of the rotor blade

\subsection{Bending Moments}

It was assumed that the forces acted at the $1 / 4$-chord, so there was no moment around the $\mathrm{x}$-axis. The magnitude of the moment about the $\mathrm{y}$-axis was small due to the relatively small drag force that produced it. Figure 4.20 shows that the $y$-axis moment distribution attained a maximum value of less than $0.2 \mathrm{Nm}$. 


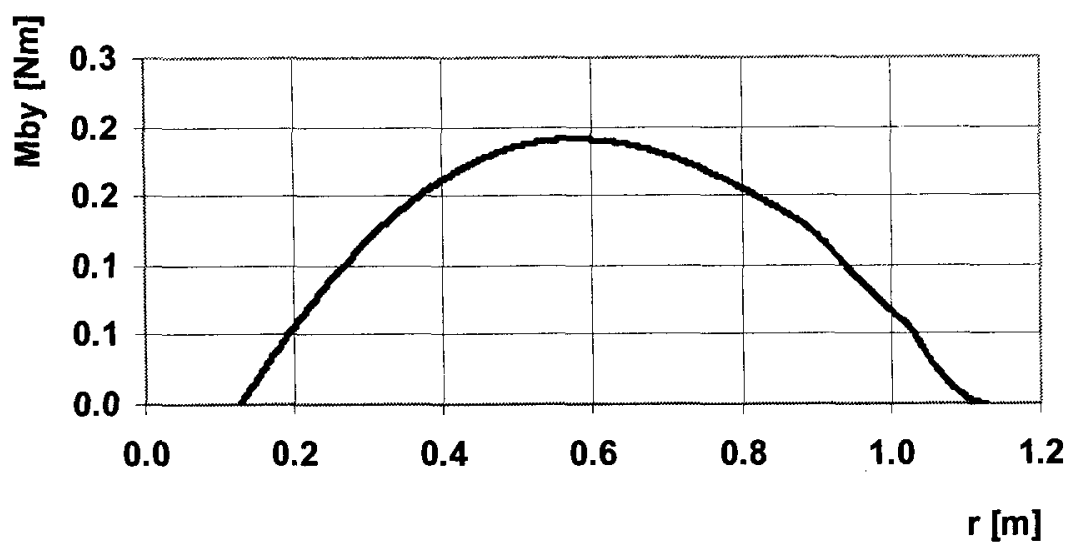

Figure 4.20: Bending moment about the y-axis along the span of the rotor blade

For the bending moment about the z-axis, the moments from the static and the dynamic loads were superimposed and the maximum value at each radial location was used for the calculation of the safety factor. Figure 4.21 shows the static and flapping $z$-axis bending moment distribution with the maximum moment along the span shown in Figure 4.22.

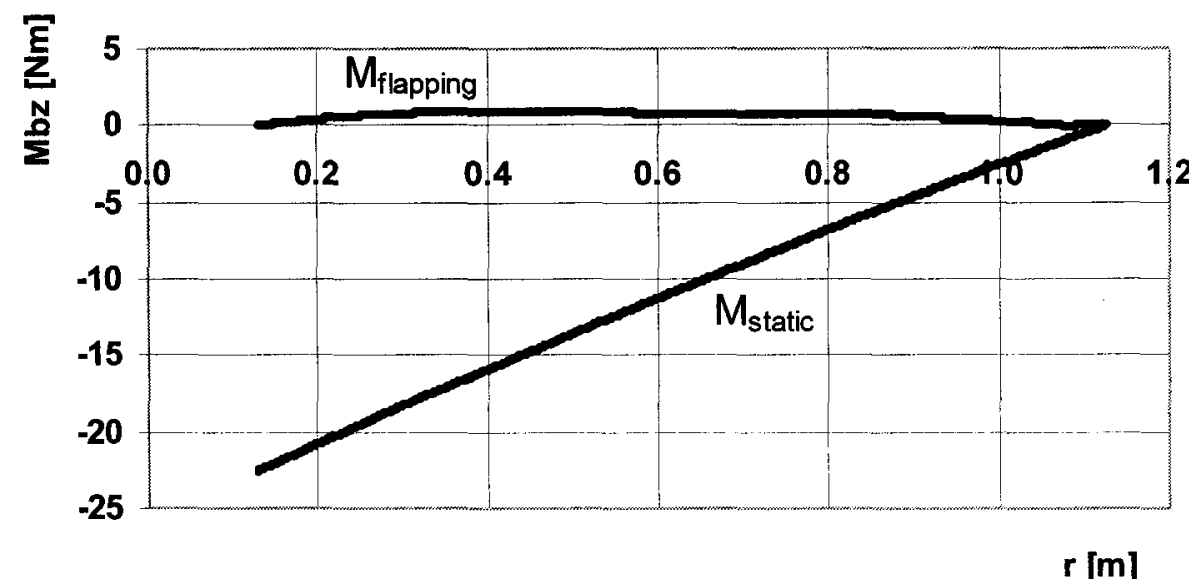

Figure 4.21: Flapping and static moments about the z-axis along the span of the rotor blade 


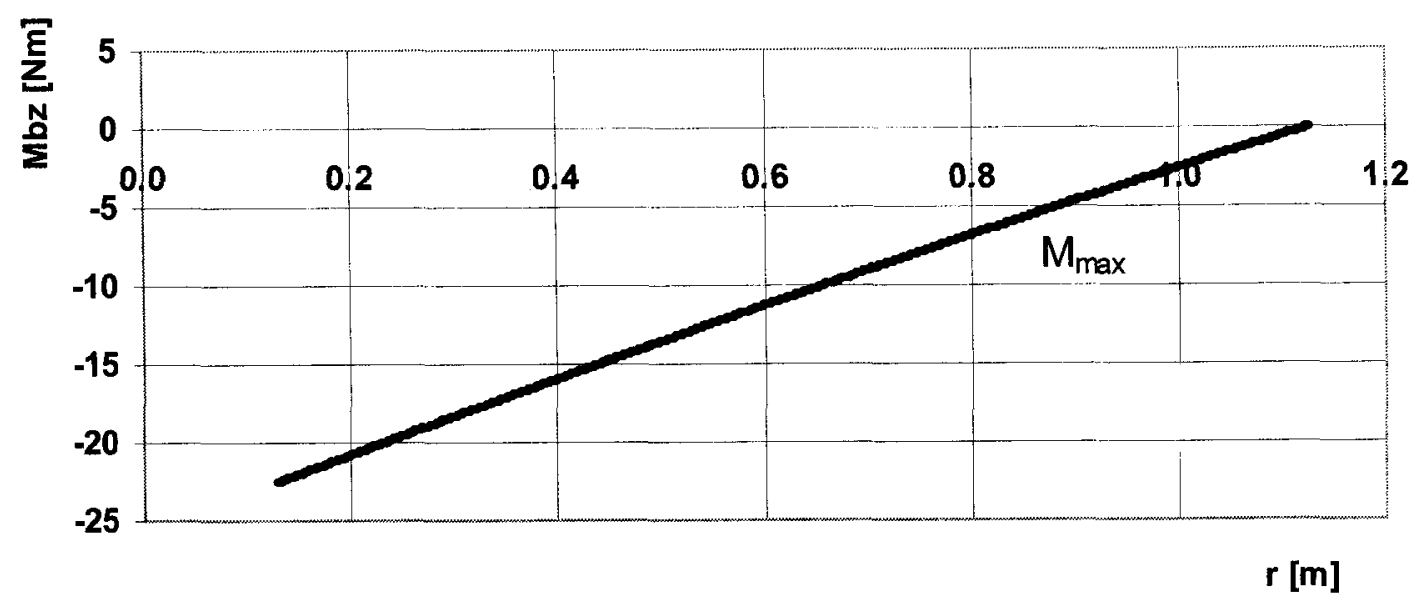

Figure 4.22: Maximum magnitude $\mathrm{z}$-axis moment along the span of the rotor blade

\subsection{Local Loads}

With the SHARCS actuated systems integrated into the rotor blade, various local loads would arise and need to be taken into account. As the actuation systems have not been completed at the time of this work, the actuator and hinge loads were not included. Another reason for this was that these loads would require local strengthening of the support structure and its design cannot proceed before that for the overall blade has been established. Therefore these effects are left for the detailed design phase of the project.

With the loads acting on the rotor blade calculated, the stress analysis can now be conducted. In order for the laminate analysis to be performed, the global stresses of the structure need to be determined. 


\section{CHAPTER 5 STRUCTURAL ANALYSIS}

\subsection{Introduction}

With the rotor blade loads established, the stresses in the structure and in turn, the stresses in the laminae of the composite need to be calculated. To calculate the overall structural stresses, elementary bending and torsion theories were employed together with some simplifying assumptions for thin-walled sections. Due to the inhomogeneous nature of the sandwich structure of the rotor blade, the concept of an equivalent homogeneous cross-section was used to simplify the analysis of the structure. To check the structural integrity of the composite structure, the failure criteria based on fibre failure as well as inter-fibre failure [Puck 2002] were employed. All the steps and analysis procedures described herein follow closely to those developed at DLR [Möller 2004].

\subsection{Structural Loads}

The main load that the rotor blade experiences was the tensile axial stress $\left(\sigma_{x}\right)$ caused by the centrifugal force on the rotor blade. Two structural configurations were deemed possible for the final design of the cross-section that carries the tensile stress. There 
could be a C-spar, where the laminate lay-up is thicker at the nose, or a nose spar occupying the volume directly behind the leading edge of the profile, or a combination of both as shown in (Figure 5.1). The following steps were taken in the stress analysis of each of these designs.

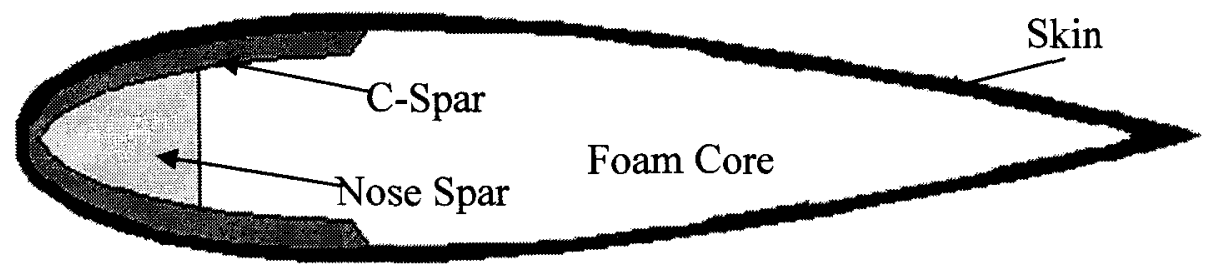

Figure 5.1: Schematic rotor blade cross-section with C-spar

When subjected to a load, the stresses at the spar and along the skin would not be uniform due to the different thicknesses, materials and lay-ups that may be used. First, an equivalent homogeneous structure was developed with constant span-wise Young's modulus. To achieve this equivalent homogeneous structure, the skin thickness was changed to maintain the same structural rigidity as the original structure, while obtaining the constant Young's modulus required. For simplicity, the centre-line for the profile was maintained in the equivalent homogeneous structure. Figure 5.2 shows the schematic of the resulting cross-section of the structure; the actual profile depends on the Young's modulus required, and may result in either an overlap or gap between the spar and skin. This feature has no meaning in reality as the homogeneous cross-section was only used for the determination of the preliminary geometric dimensions. 


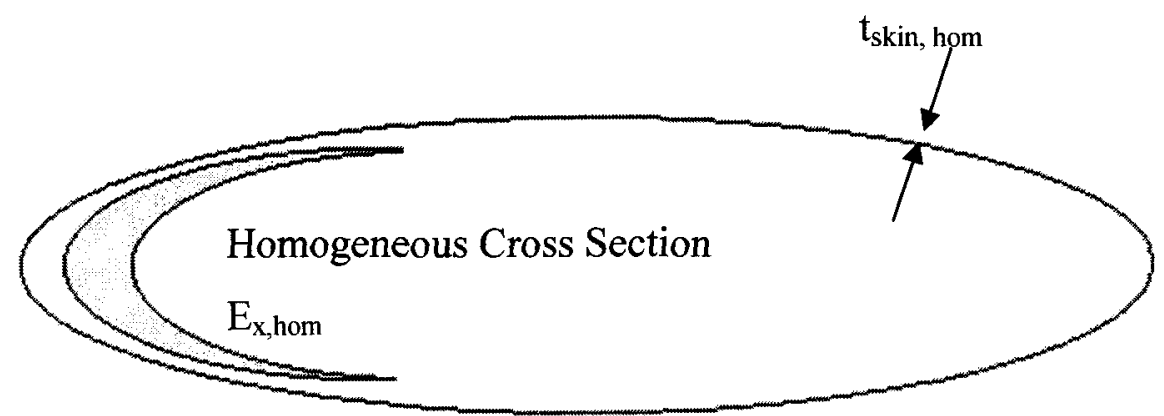

Figure 5.2: Schematic homogeneous rotor blade cross-section

The homogeneous Young's modulus $\left(E_{x, h o m}\right)$ can be arbitrarily chosen, and for convenience, that corresponding to the spar was selected. The resulting skin thickness of the homogeneous structure $\left(t_{\text {skin,hom }}\right)$ could therefore be obtained as follows:

$$
t_{s k i n, \mathrm{hom}}=t_{s k i n} \cdot \frac{E_{x, \text { skin }}}{E_{x, \mathrm{hom}}}
$$

where: $t_{s k i n}$ is the skin thickness, and $E_{x, \text { skin }}$ is the Young's modulus in the fibre direction for the non-homogeneous structure. The homogeneous longitudinal stress $\left(\sigma_{x, h o m}\right)$ over the homogeneous spar cross-section at any station along the blade span can be approximated from the force in the $\mathrm{x}$ direction $\left(\mathrm{Q}_{\mathrm{x}}\right.$, Section 4.7) and the cross-sectional area of the homogeneous spar $\left(A_{\text {hom }}\right)$.

$$
\sigma_{x, \text { hom }}=\frac{Q_{x}}{A_{\text {hom }}}
$$


From compatibility the strain $\left(\varepsilon_{x}\right)$ of the spar must be equal to the strain of the skin; thus, the stress of the skin $\left(\sigma_{x, \text { skin }}\right)$ could be calculated as follows.

$$
\varepsilon_{x}=\frac{\sigma_{x, \text { Skin }}}{E_{x, \text { Skin }}}=\frac{\sigma_{x, \text { hom }}}{E_{x, \text { hom }}}
$$

Substitution of (5.2) into (5.3) provides the skin stress under the primary load at each point (i) along the span of the blade.

$$
\sigma_{x, \text { skin }, i}=\frac{Q_{x} \cdot E_{x, i}}{A_{\mathrm{hom}} \cdot E_{x, \mathrm{hom}}}
$$

The resultant centrifugal force acts at the centre of mass of the cross-section. With the equivalent homogeneous cross-section, this would not generally be at the same location in space as the actual cross-section. Thus the primary load, $Q_{x}$, of the cross-section acts through a different axis, and not along the origin ( $\mathrm{x}$-axis), thereby, causing an additional bending moment about the y-axis. The resulting moment was calculated as in Eq. (5.5). This quantity was then added to the moment calculated previously in Chapter 2 .

$$
M_{b y, Q x}=Q_{x} \cdot\left(z_{P}-z_{P, H o m}\right)
$$

where: $z_{P}=\mathrm{Z}$-co-ordinate of the centre of mass in the airfoil coordinate system $z_{P, H o m}=$ Z-co-ordinate of the centroid of the homogeneous geometry 


\subsection{Shear Force}

In thin-walled sections under a transverse load, the shear flow can be assumed to be constant across the thickness and is taken to be tangential to the centre-line of the structure. The schematic for the structure centre line and shear flow direction are shown in Figure 5.3. The shear flow along the wall of the section was taken to be a superposition of a uniform shear flow $q_{o}$ in a closed-section and the shear flow in an open-section as shown in the figure.

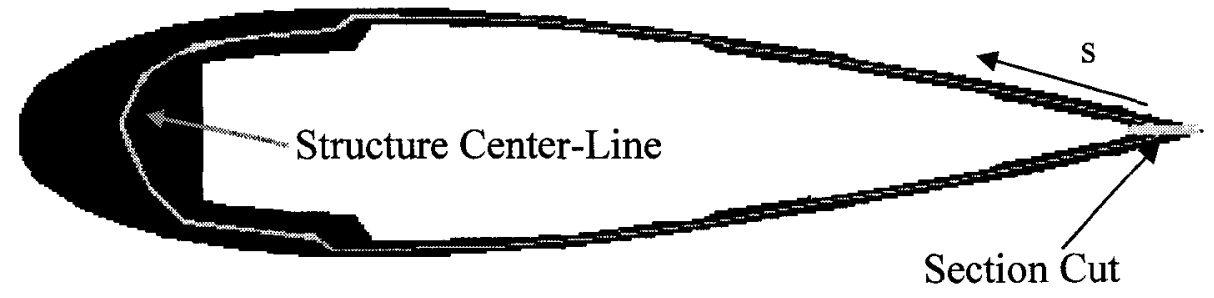

Figure 5.3: Structure centre-line

An imaginary cut was made in the section so that the shear flow at the cut was zero, which provides a starting location for the shear flow calculation. For the homogeneous cross-section the total shear flow was given by:

$$
q(s)=-\left(Q_{\hat{y}} \cdot \frac{S_{\hat{z}}(s)}{I_{\hat{z}}}+Q_{\hat{z}} \cdot \frac{S_{\hat{y}}(s)}{I_{\hat{y}}}\right)+q_{0}
$$

with: $S=$ first moment of area

$I=$ second moment of area 
The constant shear flow $\mathrm{q}_{0}$ due to the effective torque, was obtained using the following equation:

$$
q_{0}=\frac{1}{2 \cdot A_{0}} \cdot\left[Q_{\hat{z}} \cdot\left(y_{M_{c}}-y_{M}\right)-Q_{\hat{y}} \cdot\left(z_{M c}-z_{M}\right)\right]
$$

where: $A_{0}$ is the enclosed area of the cross-section of the mid-thickness line around the perimeter and $y_{M}$ and $z_{M}=\mathrm{y}$ and $\mathrm{z}-$ co-ordinate of the shear centre in the open section $y_{M c}$ and $z_{M c}=\mathrm{y}$ and $\mathrm{z}-$ co-ordinate of the shear centre in the closed section

As the shear flow arises from the change in the primary stress along the blade span, it was convenient to set the arbitrary Young's modulus of the homogeneous cross-section to the value of the spar. Thus the origin of the co-ordinate system of the required equivalent homogeneous cross-section acts in the centroid of the original cross-section. The directions of the other two axes were therefore parallel to the original non-homogeneous axes, due to both the defined geometric and flexibility symmetry. This makes it unnecessary to transform in the new co-ordinate system the other transverse loads applied to the blade $\left(Q_{y}\right.$, and $\left.Q_{z}\right)$, as they would be correct in both magnitude and direction in both systems. However, as with the primary stress, an additional moment may result due to the different cross-section that must be considered. 
From symmetry, the shear centre must be on the chord line, so the z-axis transverse force would not cause a torsional moment. The other transverse force $\left(Q_{y}\right)$ acts at the $1 / 4$-chord line, which may not be at the same location as the shear centre. That results in a torsional moment $\left(M_{b x, Q y}\right)$, given by the following equation.

$$
M_{b x, Q y}=Q_{y} \cdot\left(z_{P}-\frac{c}{4}\right)
$$

with $z_{P}=\mathrm{z}$-co-ordinate of the shear centre in the non-homogeneous airfoil coordinate system

Two further simplifications were used for the shear flow calculations using the open section thin walled theory. The first was the fact that the nose section was thicker than the rest of the blade, and the thin-walled theory may not be applicable in this region. As the spar region was ahead of the $1 / 4$-chord line, most of the structure was valid for thinwalled theory. In the thicker spar section, the shear stress varies across the thickness, but for simplicity, it was assumed to be uniform and with magnitude corresponding to the largest value there. This means in the nose area, the shear flow calculated would be higher than in reality, resulting in a conservative design.

With regard to the thin walled requirement, the calculated shear flow from the open section formula assumes that the shear of an infinitesimal area transfers the entire shear to the neighboring elements. This is accurate if the element has a constant stress. To ensure 
that the thin walled shear flow calculations would be accurate, the cross-section was discretized into rectangular elements (Figure 5.4). The rectangles have a constant material thickness enclosed and their centroids lay on the centre-line of the skin. Thus the moment caused by the shear flow of the homogeneous cross-section was equal to the moment for the actual design.
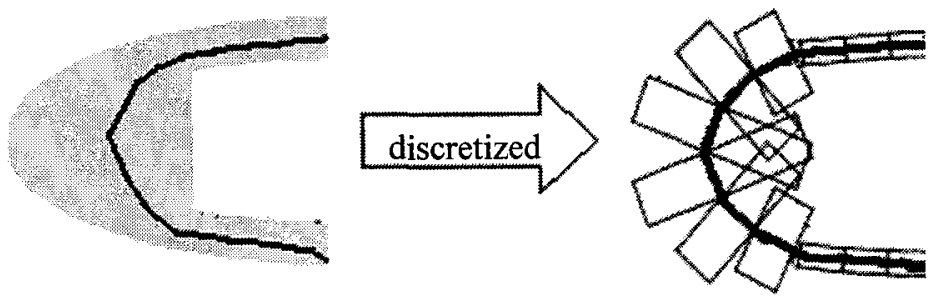

Figure 5.4: Schematic of rotor blade leading edge discretization

\subsection{Shear Centres}

In order to calculate the shear flow described in Section 5.2, the shear centres of both the open $\left(y_{M}\right.$ and $\left.z_{M}\right)$ and closed section $\left(y_{M c}\right.$ and $\left.z_{M c}\right)$ were required. The shear centres of the open section, with the cut at the trailing edge, were calculated in the homogeneous coordinate system using the following equations:

$$
\begin{aligned}
& y_{M}=\frac{1}{I_{\ddot{y}}} \cdot \int_{s_{\text {start }}}^{s_{\text {end }}} S_{\hat{y}}(s) \cdot r_{t}(s) \cdot d s \\
& z_{M}=\frac{1}{I_{\hat{z}}} \cdot \int_{s_{\text {start }}}^{s_{\text {end }}} S_{\hat{z}}(s) \cdot r_{t}(s) \cdot d s
\end{aligned}
$$


where: $r_{t}$ is the perpendicular distance from the moment centre to the tangent of the skin at location $s$. The shear centres of the closed section were calculated using the equation requiring the angle of twist of the cross-section to be zero, $(\theta=0)$, i.e.

$$
\theta=\frac{1}{2 \cdot A_{0} \cdot G} \cdot \oint \frac{q(s)}{t(s)} \cdot d s=0
$$

This equation was based on a homogeneous structure, which was not the case for the SHARCS rotor blade. Although the equivalent homogeneous cross-section was created using a constant Young's modulus, the shear modulus $(G)$ of the cross-section remained anisotropic; this was an accepted inconsistency of the purpose of the present work. Each different element would thus have a different shear modulus, and the requirement for zero twist becomes:

$$
\theta=\frac{1}{2 \cdot A_{0}} \cdot \oint \frac{q(s)}{t(s) \cdot G(s)} \cdot d s=0
$$

which reduces to:

$$
\oint \frac{q(s)}{t(s) \cdot G(s)} \cdot d s=0
$$


Substituting the shear flow equation (5.6), into equation (5.11) produced the following expression.

$$
\oint \frac{\left(Q_{\hat{y}} \cdot \frac{S_{\hat{z}}(s)}{I_{\hat{z}}}+Q_{\hat{z}} \cdot \frac{S_{\hat{y}}(s)}{I_{\hat{y}}}\right)+q_{0}}{t(s) \cdot G(s)} \cdot d s=0
$$

Assuming that the material remains linear elastic, the superposition principle could be applied. This allowed the separate investigation of the transverse force $\left(Q_{y}\right.$ and $\left.Q_{z}\right)$ effects.

$$
\oint\left[\left(Q_{\hat{y}} \cdot \frac{S_{\hat{z}}(s)}{I_{\hat{z}}}\right)+q_{0} \cdot\right] \frac{d s}{t(s) \cdot G(s)}=0
$$

From the moment balance in the equivalent homogeneous co-ordinate system between the shear flow and the $Q_{z}$ force, the constant shear flow term $q_{0}$ could be obtained as follows;

$$
q_{0}=\frac{Q_{z}}{2 \cdot A_{0}} \cdot\left(y_{M c}-y_{M}\right)
$$

Substituting (5.14) into (5.13) and eliminating $Q_{y}$ results in the y - co-ordinate of the position of the shear centre for the closed cross-section. 


$$
y_{M c}=y_{M}+\frac{2 \cdot A_{0}}{I_{\hat{y}}} \cdot \frac{\oint \frac{S_{\hat{y}}(s)}{t(s) \cdot G(s)}}{\oint \frac{1}{t(s) \cdot G(s)}}
$$

The $\mathrm{z}$ - co-ordinate can be similarly obtained from:

$$
z_{M c}=z_{M}+\frac{2 \cdot A_{0}}{I_{\hat{z}}} \cdot \frac{\oint \frac{S_{\hat{z}}(s)}{t(s) \cdot G(s)}}{\oint \frac{1}{t(s) \cdot G(s)}}
$$

Even though the above procedure applies only to hollow bodies, due to the negligible rigidity of the foam, the structure was assumed to behave to be so. Thus, the foam was neglected in the shear flow calculations. Any effect the foam would have on the structure would be to reduce the shear flow in the structure, thus the calculations used would result in a conservative estimate. In reality, however, the actual position of the shear centre is affected by the presence of the foam core. This is because most of the volume that the foam occupies lies behind the assumed $1 / 4$-chord shear centre, it has an effect, albeit not very large, on the moment balance about the shear centre. The actual shear centre location would be further aft of $1 / 4$-chord. According to Möller (2004), DLR designed a rotor blade using a similar method and compared the calculated shear centres with those 
from a FEA model of the rotor blade. A maximum difference of approximately $10 \%$ was found between the two shear centre values, depending on the rigidity of the foam.

From aeroelastic analysis, the $10 \%$ deviation between calculated and actual position of the shear centre may result in an unstable rotor blade [Möller 2004]. Therefore, any flutter analysis must use the value for the shear centre obtained from a finite element model, and not the calculated value. For the purpose of determining the stresses however, the effects of the possible $10 \%$ error in the position of the shear centers are not very significant indeed. The calculated shear centre lies either on or ahead of the $1 / 4^{-}$ chord. As such, the calculated twisting moment from the $Q_{y}$ transverse force would be greater than in practice. This therefore would again result in a more conservative design.

\subsection{Bending Stress}

To evaluate the bending strain, the homogeneous span-wise Young's modulus $\left(E_{x, H o m}\right)$ was revisited. In the earlier assumption, the Young's modulus was taken to correspond to that of the actual spar. This effectively gives the bending strain of the spar directly. With the bending moments already being in the homogeneous co-ordinate system, no coordinate system transformations were required either. The bending stress for the spar $\left(\sigma_{x, H o m, b, s p a r}\right)$ was then calculated using the simple bending formula: 


$$
\sigma_{x, \text { Hom }, b, s p a r}=\frac{\hat{z} \cdot M_{b y}}{I_{\bar{y}}}+\frac{\hat{y} \cdot M_{b z}}{I_{\hat{z}}}
$$

The bending stress of the skin was calculated in a similar procedure.

$$
\sigma_{x, \text { Hom }, b, \text { skin }}=\frac{E_{x, i}}{E_{x, \text { hom }}}\left(\frac{\hat{z} \cdot M_{b y}}{I_{\bar{y}}}+\frac{\hat{y} \cdot M_{b z}}{I_{\hat{z}}}\right)
$$

\subsection{Torsional Load}

To obtain the torsional load, the thin-walled closed section single cell theory was used. With no restraint on warping of the rotor blade section, a torque results in an equivalent, constant shear flow through the cross-section. The shear flow caused by the torsion of the cross-section is given by:

$$
\mathrm{q}_{\mathrm{T}}=\frac{\mathrm{M}_{\mathrm{x}, \mathrm{T}}}{2 \cdot \mathrm{A}_{0}}
$$

With the torque $\left(M_{x, T}\right)$ obtained from that of the airfoil, $M_{b x}$ and that caused by the transverse force $Q_{y}$, as denoted by $M_{b x, Q y}$.

$$
M_{x, T}=M_{b x}+M_{b x, Q y}
$$


A critical factor in determining the torsion shear flow was the enclosed cell area as represented by $A_{0}$. The idealized structural centre-line shown in Figure 5.3, surrounds a smaller area than exists in reality, thus over-predicting the shear flow. To compensate for this, the computer program used considers only the contribution of the skin and C-spar area for the torsion calculation. The program achieved this by setting the torsional rigidity of the nose spar to zero.

\subsection{Safety Factor}

From the structural analysis, the stresses the rotor blade could be obtained, from which, the resulting stresses in each lamina could also be determined. As the lamina is not isotropic, a simple comparison of the stresses to the lamina strength would not accurately predict the safety of the design. In order to determine the strength and stability of the rotor blade, a stress-oriented safety factor was required. As the purpose of the SHARCS rotor blade is as a proof of concept for the noise and vibration reduction systems, no fatigue life calculations were required, as the blade would not be operated for an extensive amount of test hours. Therefore two failure criteria were used for the design, the fibre failure and the inter-fibre failure criteria developed by Puck.

While Tsai-Hill and Tsai-Wu are also well established, Puck's methods have been used for rotor blade composite failure analysis at DLR. As DLR has more experience 
designing rotor blades, and may be the manufacturer of the SHARCS rotor blade, Puck's method was employed for the failure analysis for the SHARCS rotor blade.

\subsubsection{Fibre Failure}

The fibre failure criterion is based on fibres in the laminae breaking under a tensile force. The physical background of the criterion is analogous to failure criterion for isotropic materials. A fibre will fail when the stress on the fibre exceeds the yield strength, thereby causing significantly reduced load-carrying capacity of the lamina. The criterion for fibre failure is a simple comparison of the fibre stress, $\sigma_{l}$, and its tensile yield strength, $F_{l t}$. Thus the safety factor against such failure is given by:

$$
j_{F F}=\frac{\left|\sigma_{1}\right|}{F_{1 t}}
$$

As the rotor blade was always in a tensile stress state due to the centrifugal load, the compression strength was not required even though for composite materials the latter value was lower than the tensile strength. 


\subsubsection{Inter-Fibre Failure}

Inter-fibre failure may occur before the fibre failure. Thus, the inter-fibre failure criterion becomes the important factor in the design of a structurally safe rotor blade. This is especially true for rotor blades due to the high centrifugal stresses. The high centrifugal load particularly affects the $\pm 45^{\circ}$ plies, as the force does not act parallel to the fibre direction.

Inter-fibre failure occurs when the matrix between the fibres becomes overloaded, resulting in a local matrix crack. The crack propagates until it meets a fibre that runs transverse to the crack. In most situations, the energy release during this matrix crack propagation is not sufficient to cause fracture of the substantially stronger fibre. For composite structures, a crack-arrest fibre usually occurs within a short distance of the crack initiation point. Even with the crack growth stopped, the shear flow would no longer be transmitted over the crack surface, resulting in the load being carried by the neighbouring uncracked area, causing the fibres and matrix there to experience a higher stress.

The local failure would remain just a local feature, but an accumulation of these local failures results in a reduction in structural strength and stiffness. The reduction of the stiffness of the structure causes a decrease in its natural frequencies. For a rotor blade, this may result in flutter as a decreased natural frequency may reside in a region of 
exciting forces that are now in phase with the natural frequency. A serious concern in this regard was that large distances could separate the crack arresting fibres or they may not exist in the region of crack propagation. This means that the required safe shear flow transfer becomes more difficult, the longer the crack becomes. According to Puck (2002), a minimum of 3 different plies was required to ensure sufficient damage tolerance in relation to inter-fibre failures.

To determine the effect inter-fibre failures on the rotor blade structure, three different failure modes (A, B and C) related to the magnitude and direction of the transverse fibre stress were identified, as shown in Figure 5.5. For mode $A$ in the transverse tension range, a matrix crack runs perpendicular to the load direction, illustrated in Figure 5.5(a). This mode can be avoided by force diffusion to other fibres in the adjoining plies, allowing the component to remain structurally sound.

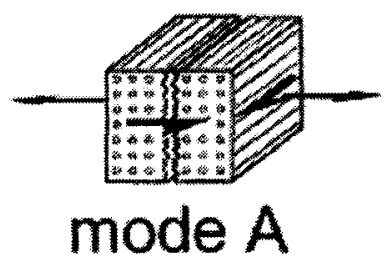

(a)

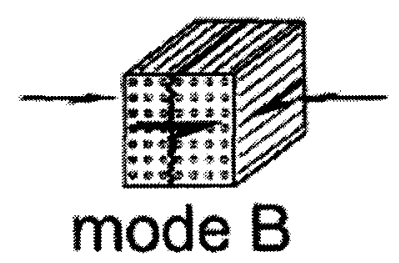

(b)

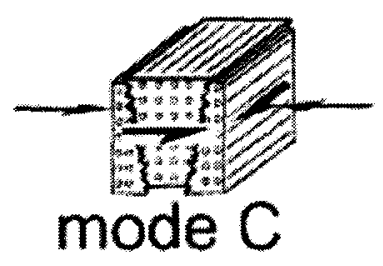

(c)

Figure 5.5: Three inter-fibre failure modes [Puck 2002]

The most serious mode to the integrity of the structure is mode C. It occurs in the perpendicular compression stress region, causing wedge-like surface fracture occurring 
diagonally to the load direction due to shear, as shown in Figure 5.5(c). This results in a laminate debonding as shown in Figure 5.6, which leads to component failure; it was therefore the critical inter-fibre failure mode.

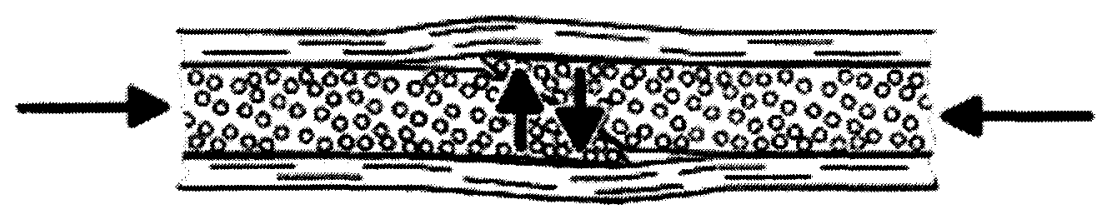

Figure 5.6: Debonding effect of mode C [Puck 2002]

Mode B occurs in the moderate compression load, perpendicular to the fibre direction, illustrated in Figure 5.5(b). However in this mode, no wedge was formed, only a perpendicular crack. With increasing compression load, the crack angle changes from $90^{\circ}$ to $45^{\circ}$ to the load direction. When the angle reaches $45^{\circ}$, mode $\mathrm{B}$ becomes mode $\mathrm{C}$. Similar to mode A, the transfer of force to neighbouring fibres allows the structure to tolerate the damage associated with this mode.

Figure 5.7 [Möller 2004] show the general schematic failure curve of the lamina when the fibre stress $\sigma_{1}$ is zero, with $F_{2 c}, F_{2 t}$ and $F_{6}$ being the strength values, obtained from the pre-preg manufacturer, for perpendicular compressive stress, perpendicular tensile stress, and shear stress respectively. The loci constants $a, c$, and $d$ are related to the strength values of the composite pre-preg material at $\sigma_{l}$ equal to zero, and represent the ellipse radius along the $\sigma_{2}$-axis, the radius along the $\tau_{21}$-axis, and the distance from the 
$\tau_{2 I}$-axis to the centre of the ellipse, respectively. The solid curve shows the failure values from experiment, while the dashed curve shows the idealized curve. In the perpendicular compressive region, the idealized failure curve was conservative in the calculated failure when compared to experimental curve. This feature helps in adding an extra degree of conservative design in the mode $\mathrm{C}$ failure region.

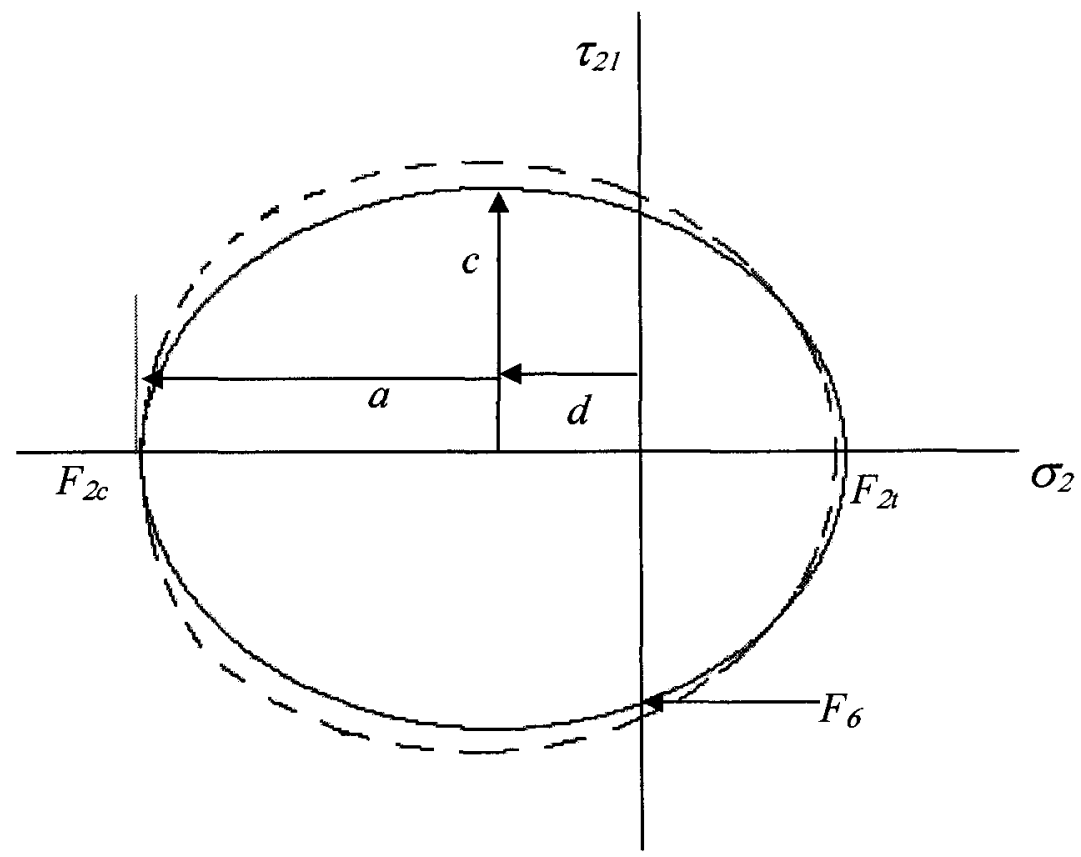

Figure 5.7: Failure locus for zero fibre stress [Möller 2004]

In the perpendicular tension stress region, the idealized curve slightly over estimates the stress required for failure. To combat this, a required safety factor $\left(j_{\min }=1.2\right)$ was applied to the calculation. As it could not be ensured that the final rotor blade design would have the required 3 different ply angles at all locations, all 3 inter-fibre failure modes were treated as critical failures. 


\subsubsection{Failure Loci for Inter-Fibre Failure}

The failure ellipse shown in Figure 5.7 is symmetric along the $\sigma_{2}$-axis. In order to determine the idealized ellipse when $\sigma_{l}=0$, the uni-directional ply strengths $\left(F_{2 t}, F_{2 c}\right.$ and $\left.F_{6}\right)$ were required, which could be obtained from the manufacturer of the pre-preg.

The tensile stress state of the rotor blade is, however, not represented in Figure 5.7. Thus the manufacturer supplied strengths of the uni-directional pre-pregs would be reduced with increasing stress $\sigma_{l}$. From Puck (1996) it was found that the failure ellipse remains relatively unchanged up to $70-75 \%$ of $F_{1 t}$ after which a noticeable reduction in the ellipse occurs. At this parallel fibre strength, the perpendicular and shear strengths were reduced to $50 \%$ of their original values. In order to apply the inter-fibre failure criterion, this effect was approximated using ellipsoids. This was done by creating an $F_{1}{ }^{*}$ that was the intercept point for the ellipse on the parallel stress axis that resulted in an ellipse with perpendicular and shear strength values of $50 \%$ at the parallel strength point, as shown in Figure 5.8. 


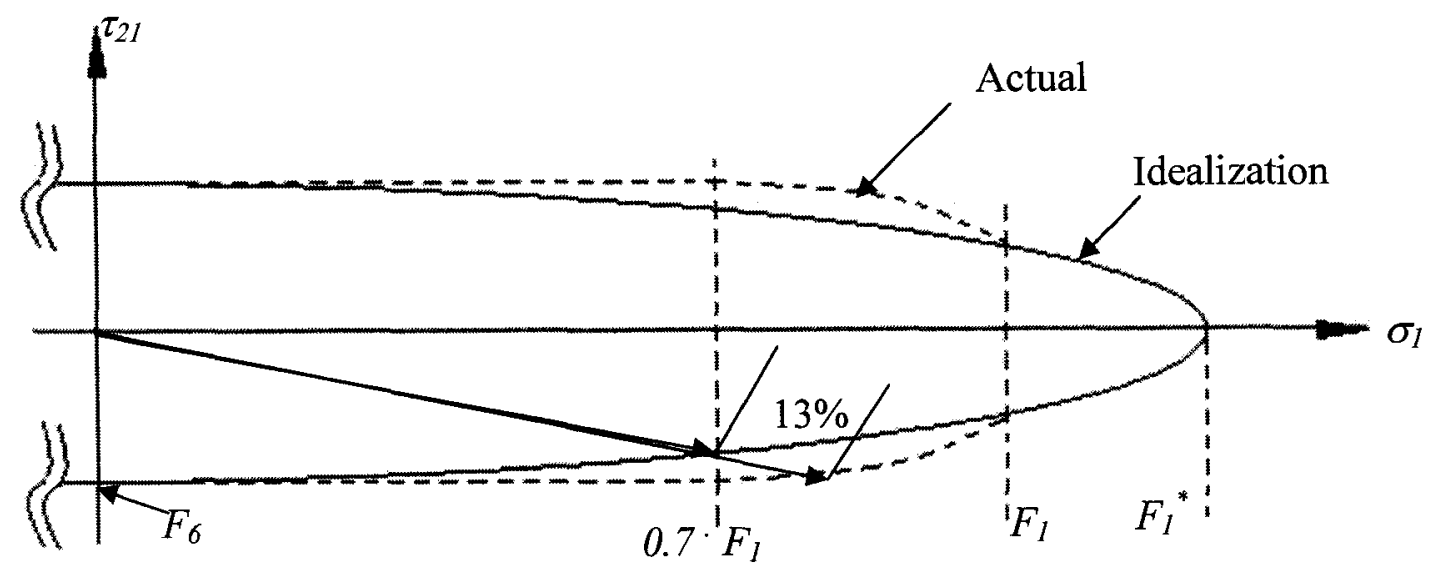

Figure 5.8: Idealized and actual failure ellipse in the parallel and shear stress axes

As the idealized ellipse occurs inside the actual ellipse for large portions along the parallel stress axis, a conservative design approach was maintained. The maximum deviation between this actual and idealized ellipse occurred at the fiber-parallel stress of $\sigma_{l} \approx 0.7 \cdot F_{l}$ and was found to be $13 \%$ conservative. If the safety factor was greater then 1.3, all tensile states occur below $0.7 \cdot F_{l}$. Therefore, the resulting error between the actual and idealized would be minimized.

In contrast to Figure 5.8, was the failure ellipse in the perpendicular and parallel stress plane, because the perpendicular tension and compression strengths of the lamina are not symmetric to the $\sigma_{1}$-axis. With that, a skewed ellipse was produced and is illustrated in Figure 5.9. 


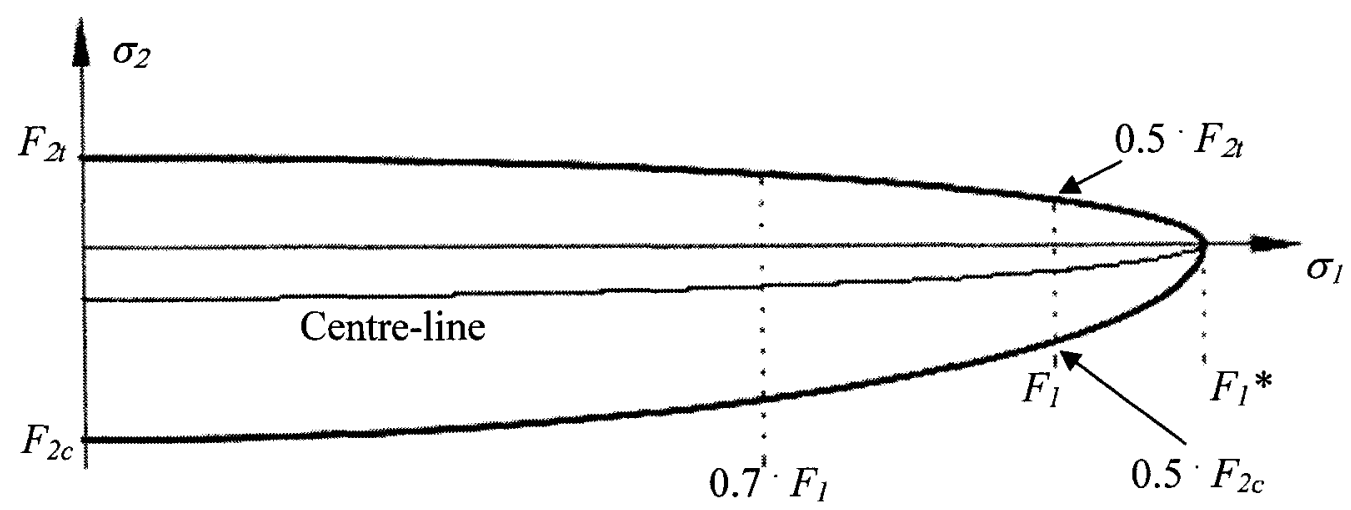

Figure 5.9: Fibre failure ellipse in the parallel and perpendicular stress plane

Combining the previous ellipses resulted in a 3-D asymmetric ellipsoid known as the "failure banana". Figure 5.10 shows the failure banana developed for the inter-fibre failure criterion. It is clearly seen that the fibre failure criteria must be examined separately, as it cuts through the failure banana at the tip at a value of $F_{l}$.

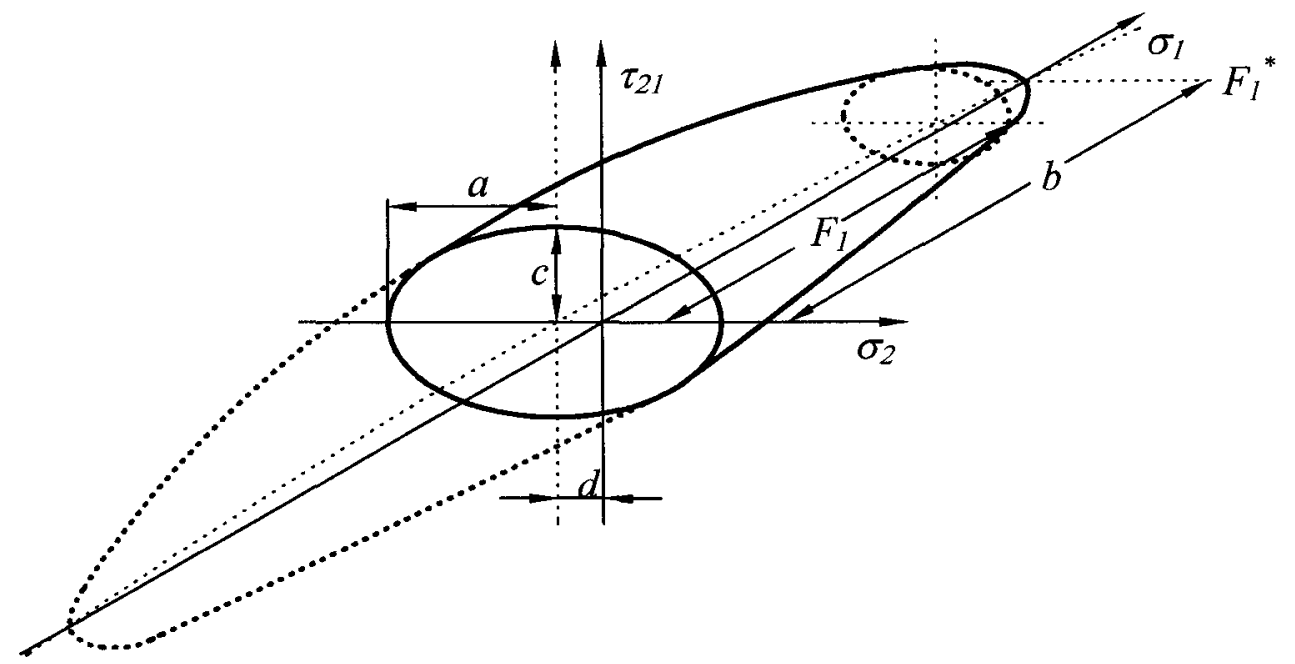

Figure 5.10: Failure Banana [Möller 2004]

Now, any stress state could be found in the $\sigma_{z} \tau_{2 \Gamma} \sigma_{l}$ co-ordinate system, and compared to the failure banana. If the stress state occurred within the bounds of the failure banana, 
there would be no inter-fibre failure. The inter-fibre failure safety factor was then defined as the value that when multiplied with the vector of the stress quantities result in a value that passes through the failure banana.

\subsubsection{Mathematical Representation of the Failure Banana}

From Figure 5.11, with the ellipse shifted by $d$ from the $\sigma_{2}$ axis, the 3-D ellipsoids in the $\sigma_{\tau} \tau_{2 \Gamma} \sigma_{l}$ co-ordinate system were characterized by the following equation.

$$
\left(\frac{\sigma_{2}+d}{a}\right)^{2}+\left(\frac{\sigma_{1}}{b}\right)^{2}+\left(\frac{\tau_{21}}{c}\right)^{2}=1
$$

In order to obtain the failure banana form the constant axis offset of $d$ was changed to the $\sigma_{l}$ dependant value $d^{*}$ to account for the change in the ellipse centre position which varies along the $\sigma_{1}$-axis, shown previously in Figure 5.9 as the centre-line. Figure 5.11 shows that $d^{*}$ produces the required elliptical curve. 


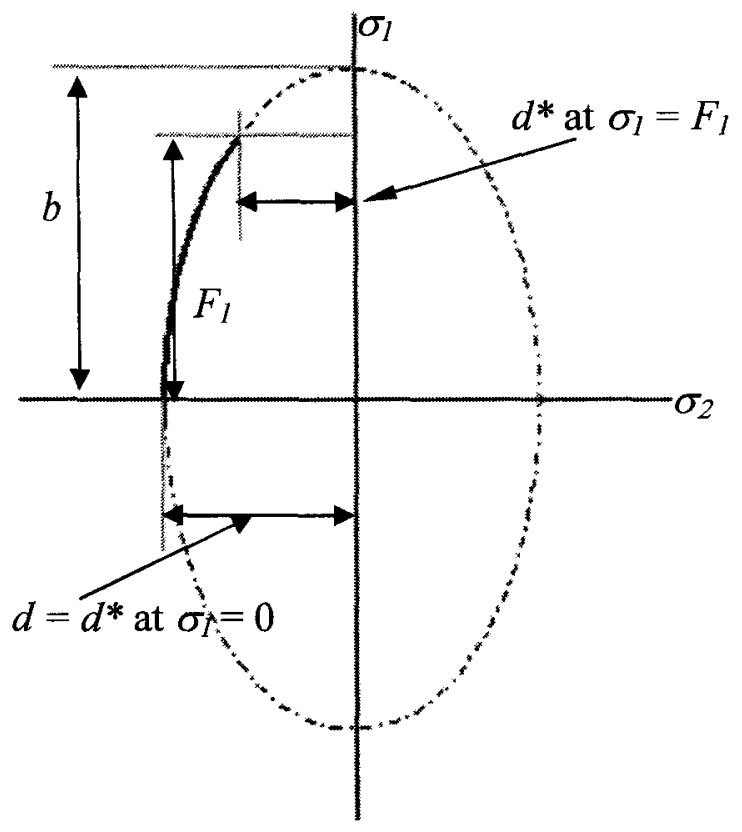

Figure 5.11: $d^{*}$ ellipse

Constants for the equation ( $a, b, c, d$ and $\left.d^{*}\right)$ were all obtained from the uni-directional pre-preg strength values $\left(F_{1}, F_{2 t}, F_{2 c}, F_{6}\right)$.

$$
\begin{aligned}
& a=\frac{F_{2 t}+F_{2 c}}{2} \\
& d=a-F_{2 t}=\frac{F_{2 c}-F_{2 t}}{2}
\end{aligned}
$$

Next, substituting $\sigma_{l d}$ for $\sigma_{l}$, and $d$ for the intercept results in the 2-D ellipse equation. 


$$
\left(\frac{d^{*}}{d}\right)^{2}+\left(\frac{\sigma_{1 d}}{b}\right)^{2}=1
$$

Rearranging, the equation that describes the change of the centre of the ellipse was obtained to be as follows:

$$
d^{*}=d \cdot \sqrt{1-\left(\frac{\sigma_{1 d}}{b}\right)^{2}}
$$

Thus, the parallel fibre stress required to obtain $d^{*}$ was dependant on $\sigma_{l d}$ at that respective intercept. Therefore an analytical expression for $\sigma_{1 d}$ that was dependent on $\sigma_{2 d}$ was required. The extra equation for the calculation of the intercept between the vector representing the stress state and the failure banana results in a fourth degree polynomial. Due to the large number of additional calculations required to solve, an iterative approach was chosen.

The origin shift $d^{*}$ was first calculated by assuming $\sigma_{l d}=\sigma_{1}^{*} 1.3$, and calculating the intercepts. A consequence of this assumption was that the calculated inter-fibre failure would not result in the safety factor of 1.3 , as the equation would deviate slightly from the theoretical solution shown previously in Figure 5.10. The relative error between the calculated safety factor becomes larger as the calculated value deviates from 1.3 , which was thus acceptable. 
Using an iterative approach, a more accurate approximation was obtained by using the initial estimate for the inter-fibre failure safety factor for each case along the $d^{*}$ origin line. The value for $c$ was calculated by rearranging the ellipse equation as follows:

$$
\begin{aligned}
& \left(\frac{\sigma_{2}+d}{a}\right)^{2}+\left(\frac{\tau_{21}}{c}\right)^{2}=1 \\
& c=\frac{\left|\tau_{21}\right|}{\sqrt{1-\left(\frac{\sigma_{2}+d}{a}\right)^{2}}}
\end{aligned}
$$

Substitution of $\left(\left(\sigma_{2}=0 ; \tau_{2 I}=F_{6}\right)\right.$ and using the values of the constants $a$ and $d$ results in:

$$
c=\frac{F_{6}}{\sqrt{1-\left(\frac{F_{2 c}-F_{2 t}}{F_{2 c}+F_{2 t}}\right)^{2}}}
$$

Using a similar process, the constant $b$ was obtained using $\sigma_{l}=F_{l}$, which resulted in:

$$
\begin{aligned}
& \left(\frac{F_{1}}{b}\right)^{2}+\left(\frac{\tau_{21}}{c}\right)^{2}=1 \\
& \tau_{21}=0.5 \cdot\left(F_{6}\right)
\end{aligned}
$$

With these quantities, the rearranged ellipse equations, Eq (5.28), would give: 


$$
b=F_{1} *=\frac{F_{1}}{\sqrt{1-\left(\frac{0.5 \cdot F_{6}}{c}\right)^{2}}}
$$

In order to calculate the inter-fibre failure safety factor, a straight-line vector $(g)$ must be defined to connect the vector representing the stress state to the failure banana. The vector representing the stress state was given by:

$$
\vec{s}=\left(\begin{array}{c}
\sigma_{2}(s) \\
\sigma_{1}(s) \\
\tau_{21}(s)
\end{array}\right)
$$

With the required straight-line vector given as:

$$
g\left(s ; \sigma_{2}\right)=\left(\begin{array}{c}
\sigma_{2} \\
\frac{\sigma_{1}(s)}{\sigma_{2}(s)} \cdot \sigma_{2} \\
\frac{\tau_{21}(s)}{\sigma_{2}(s)} \cdot \sigma_{2}
\end{array}\right)=\left(\begin{array}{l}
\sigma_{2} \\
\sigma_{1} \\
\tau_{21}
\end{array}\right)
$$

First, the equation for the straight-line vector was substituted into the 3-D ellipsoid equation, giving the following relationship for the intercept of the failure banana. 


$$
\left(\frac{\sigma_{2 d}+d^{*}}{a}\right)^{2}+\left(\frac{\frac{\sigma_{1}(s)}{\sigma_{2}(s)} \cdot \sigma_{2 d}}{b}\right)^{2}+\left(\frac{\frac{\tau_{21}(s)}{\sigma_{2}(s)} \cdot \sigma_{2 d}}{c}\right)^{2}=1
$$

Rearranging, an equation for the variable $\sigma_{2 d}$ was obtained.

$$
\sigma_{2 d}=\frac{-\left(\frac{d^{*}}{a^{2}}\right) \pm \sqrt{\left(1-\left(\frac{d^{*}}{a}\right)^{2}\right) \cdot\left(\frac{1}{a^{2}}+\left(\frac{\sigma_{1}(s)}{b \cdot \sigma_{2}(s)}\right)^{2}+\left(\frac{\tau_{21}(s)}{c \cdot \sigma_{2}(s)}\right)^{2}\right)+\left(\frac{d^{*}}{a^{2}}\right)^{2}}}{\frac{1}{a^{2}}+\left(\frac{\sigma_{1}(s)}{b \cdot \sigma_{2}(s)}\right)^{2}+\left(\frac{\tau_{21}(s)}{c \cdot \sigma_{2}(s)}\right)^{2}}
$$

It is evident that there are two possible solutions to this equation that correspond to the 2 locations the straight-line vector intercepts the failure banana. As the failure banana was asymmetric, the two solutions would have different magnitudes. The required value of $\sigma_{2 d}$ must be an extension of the stress state vector. Therefore, a definition of the different cases was required.

The denominator was determined to always be positive, leaving the numerator of the equation as the determining factor of the sign of $\sigma_{2 d}$. Figure 5.10 shows that ' $a$ ' was larger than ' $d$ '. For the first part of the numerator, the $a^{2}$ in the denominator results in a small value for the fraction. Thus, the only part of the equation that influences the sign of 
$\sigma_{2 d}$ was the discriminant in the numerator. With a positive perpendicular tensile stress resulting in a positive intercept with the failure banana, the sign of $\sigma_{2}(s)$ must be determined based on the sign of the square root. By comparing the fibre parallel compressive stress of the intercept $\sigma_{2 d}$ with the fibre parallel compressive stress of the intercept $\sigma_{2}$, an equation for the inter-fibre failure safety factor was obtained.

$$
j_{I F F}=\frac{\sigma_{2 d}}{\sigma_{2}}
$$

A special case exists when $\sigma_{2}=0$, resulting in the previous equation being undefined. From Figure 5.10, it was determine that the inter-fibre failure safety factor would become:

$$
j_{I F F}=\frac{\left|\tau_{21}\right|}{F_{6}}
$$

\subsection{Concluding Remarks}

Using the equations presented in this chapter, an iterative approach was used to obtain an acceptable design from the structural integrity standpoint. Once such a design has been developed, a finite element model (FEM) would then be developed as well to ensure that the rotating natural frequencies of the design would be in the required range. 


\section{CHAPTER 6 SHARCS ROTOR BLADE DESIGN}

\subsection{Introduction}

With the rotor blade loads, basic mechanics of composite materials and methods of structural analysis reviewed in the previous chapters, the design of the SHARCS rotor blade could proceed. The derived equations were coded into a DLR finite element computer program (Wierach (2004) and Möller (2004)) that was specifically prepared for building a scaled MBB BO 105 blade from composite materials. The properties of carbon fibre and glass fibre composites at different ply angles were the input data. With these properties, the program allows the operator to choose the type of composite and the number of plies at any station along with the profile of the rotor blade. Subsequently, the program calculates the new local flexural rigidity $E I$ and torsional rigidity $G J$, mass per unit length $m_{x}$, centre of gravity, and the safety factor for the rotor blade.

The purpose of using this program was to match $G J$ and $E I$ values with their corresponding target values (initial value obtained in Chapter 3). After several design iterations, both the $G J$ and $E I$ values converged to the target. The safety factor was also calculated at this stage of the process. The design would be considered satisfactory when 
an adequate safety factor had been obtained. As the DLR finite element program could not perform a modal analysis on the rotor blade, a separate FEA package was required. For the non-rotating cases, the chosen program was MSC.Nastran. As MSC.Nastran could not calculate rotating natural frequencies, the finite element analysis package was changed to ANSYS for the rotating cases, which possessed that analysis ability. This fact was not initially known at the beginning of this project, or ANSYS would have been used from the start.

\subsection{Composite Materials}

To start designing the rotor blade, the composite materials had to be selected and their properties entered into the computer program. The composites chosen were IM6/epoxy carbon fibre and S-glass/epoxy fibreglass with the properties given in Table 6.1 from Daniel (1994) and Tsai (1988). Fibreglass was used for the $0^{\circ}$ plies, as carbon fibre composites led to excessive stiffness, resulting in high natural frequencies. Fibreglass has lower stiffness; hence more plies could be used to provide sufficient structural thickness for the stress caused by the centrifugal loading. Although fibreglass is heavier, most of the plies would be placed ahead of the $1 / 4$-chord, thereby reducing the amount of lead ballast required for the rotor blade to achieve the correct mass distribution properties. Thus a larger fraction of the mass of the blade becomes structural, increasing the structural efficiency. 
Table 6.1: Composite material properties

\begin{tabular}{lcc}
\hline & S-glass/epoxy & IM6/epoxy \\
\hline \hline Fibre Volume Ratio $-V_{f}$ & 0.5 & 0.66 \\
Density $\left[\mathrm{g} / \mathrm{cm}^{3}\right]$ & 2.00 & 1.60 \\
$E_{1}[\mathrm{GPa}]$ & 43 & 203 \\
$E_{2}[\mathrm{GPa}]$ & 8.9 & 11.2 \\
$G_{12}[\mathrm{GPa}]$ & 4.5 & 8.4 \\
$\nu_{12}$ & 0.27 & 0.32 \\
Longitudinal tensile strength $-F_{1 t}[\mathrm{MPa}]$ & 1280 & 3500 \\
Transverse tensile strength $-F_{2 t}[\mathrm{MPa}]$ & 49 & 56 \\
Longitudinal compressive strength $-F_{1 c}[\mathrm{MPa}]$ & 690 & 1540 \\
Transverse compressive strength $-F_{2 c}[\mathrm{MPa}]$ & 158 & 150 \\
In-plane shear strength $-F_{6}[\mathrm{MPa}]$ & 69 & 98 \\
\hline
\end{tabular}

Through design iterations, the strength of the $+45^{\circ}$ and $-45^{\circ}$ uni-directional plies was found to be of particular importance, as the primary load would not act along the fibre direction. IM6 carbon fibre pre-preg was chosen due to the high strength values it possesses, which produced the largest safety factors of the design during the design iterations. As the off-axis stresses were a few orders of magnitude smaller than those produced by the centrifugal force, only one $+45^{\circ}$ and one $-45^{\circ}$ plies (hereby referred to as $\pm 45^{\circ}$ ) need to be used to provide acceptable safety factor and torsional rigidity, with the added advantage of having less weight. The $\pm 45^{\circ}$ plies had the added benefit of increasing the inter-fibre safety factor as the angled fibres serve to help arrest crack propagation. Using the lighter carbon fibre composite material also resulted in a low number of plies required and less lead ballast. Both of these composite pre-pregs could be obtained from HEXCEL. 


\subsection{Design}

Once the composite materials have been selected, the design of the ply lay-up was considered next. In order to keep the mass centre near the $1 / 4$-chord position, more composite layers were placed at the nose than at the trailing edge of the airfoil. This creates a C-shaped nose spar, which carries most of the load.

While a C-spar was used, as it produced a structural rigidity of the blade in the right range for the natural frequencies, it was important to estimate if it would be capable of handling the load. As calculated in Chapter 4, the maximum centrifugal load was found to be $7500 \mathrm{~N}$ at the blade root, from which the minimum spar area could be obtained. Using the fibreglass tensile strength $\left(F_{I t}\right)$, and an assumed fibre failure safety factor $\left(j_{\min }\right)$ of 1.5, the spar area was calculated from Eq. (6.1).

$$
A_{s p a r}=j_{\min } \cdot \frac{F_{C F, M a x}}{F_{1 t}}=1.5 \cdot \frac{7500}{1280}=8.8 \mathrm{~mm}^{2}
$$

Since this calculated value of $8.8 \mathrm{~mm}^{2}$ was such a small area, a C-spar would appear plausible and a nose spar would not be required (Figure 5.1). The C-spar would extend to about the $1 / 4$-chord position, which equates to an $18.8 \mathrm{~mm}$ length. To estimate the required ply thickness, the $18.8 \mathrm{~mm}$ length was doubled as the spar occurs at both the top and bottom of the profile. Neglecting any profile curvature and using the $18.8 \mathrm{~mm}$ as the 
length of the C-spar (Figure 6.1), for a safe spar design, a glass fibre ply thickness of 0.23 $\mathrm{mm}$ would be required. The final spar design was made up of 2 plies of $0.5 \mathrm{~mm}$, which provided more then the required $8.8 \mathrm{~mm}^{2}$ for a safe spar.
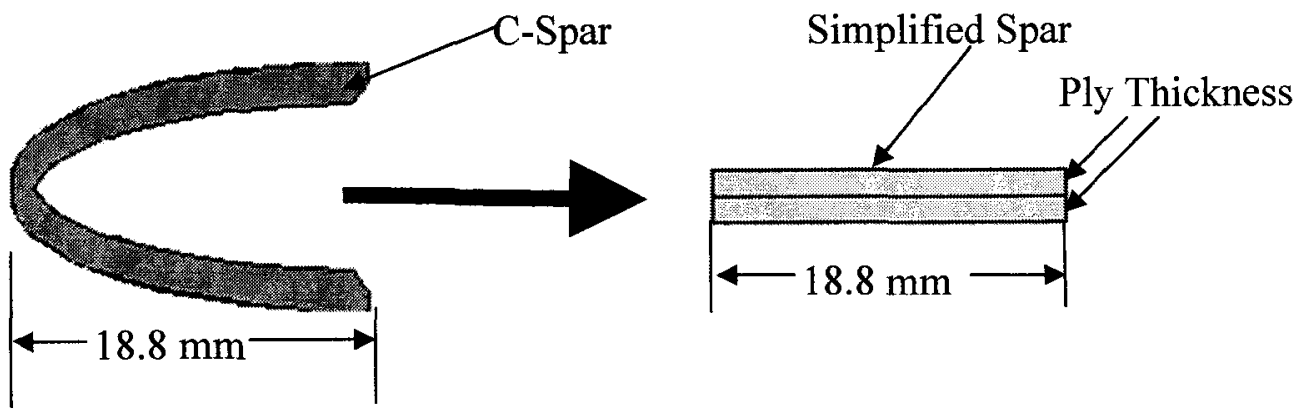

Figure 6.1: Simplified spar

Using the DLR finite element code, it was possible to calculate both the mass of the rotor blade and the centre of mass position by simply using the densities of the composite material, the thickness of the plies, and the location and length of the element for each element around the airfoil. It was found through design iteration that $170 \mathrm{~g}$ of nose lead was required to shift the centre of gravity to the required $1 / 4$-chord position. With this, the mass of the rotor blade structure (composite material, lead and foam core) was $400 \mathrm{~g}$, using a nominal foam density of $100 \mathrm{~kg} / \mathrm{m}^{3}$. This leaves an allowance of $136 \mathrm{~g}$ for the vibration and noise reduction systems if the total blade mass was not to exceed $536 \mathrm{~g}$. It should be noted that if part of the actuation system were installed ahead of $1 / 4$-chord, then the required nose ballast would decrease. This would have the benefit of increasing the amount of actuation system weight that could be used on the rotor blade before reaching the $536 \mathrm{~g}$ rotor blade weight limit. 
For this design, a minimum inter-fibre failure safety factor of 1.18 was obtained at the root of the blade, where the maximum resultant centrifugal force acts. This calculated safety factor is significantly different then that of the rough calculations for the spar as the spar calculations were simply using the fibre failure criterion, and neglecting the skin of the airfoil, which is significantly thinner then the spar. The minimum safety factor occurs in the $\pm 45^{\circ}$ plies around the chord position of $25 \mathrm{~mm}$, which is the thickest area of the airfoil resulting in the highest bending stresses. As all three of the inter-fibre failure modes were taken as critical, no calculations were performed to distinguish which of the modes was the failure mode at this location.

Due to the low safety factor, and as the blade must be attach to the hub at the root, the first $10 \%$ of the blade span was strengthened with additional $0^{\circ}$ glass fibre plies. The exact lay-up will be described in Section 6.2 .2 , but this increased the safety factor to 1.9 at the root. Thus the minimum safety factor was shifted to the $10 \%$ span line, where the strengthened root changes to the main blade lay-up. The minimum safety factor at that span position was 1.23 , due to inter-fibre failure of the $\pm 45^{\circ}$ carbon fibre plies. With that, the SHARCS rotor blade had 3 distinct lay-up regions (Figure 6.2): the root, main blade, and the anhedral tip. 


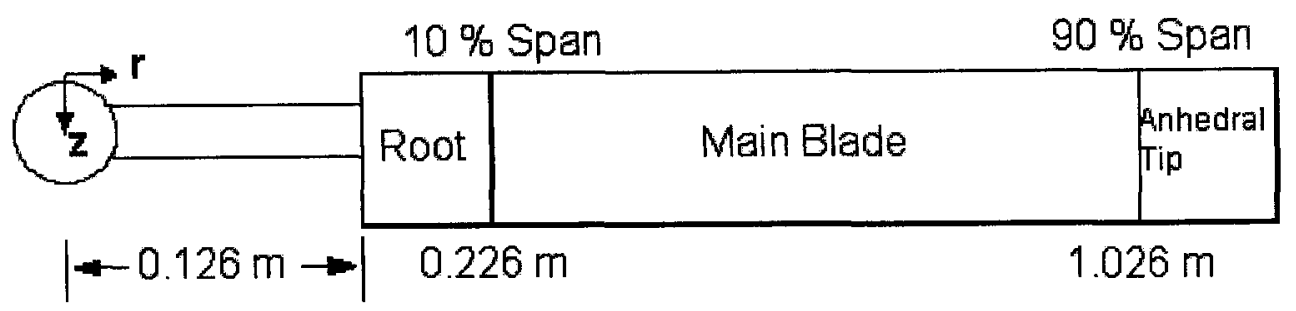

Figure 6.2: Schematic of SHARCS rotor blade sections

\subsubsection{Blade Lay-up Design}

The design for the portion of the rotor blade that occurs between $10 \%$ to $90 \%$ span is illustrated in Figure 6.3. Table 6.2 provides more detailed information for the composite lay-up.

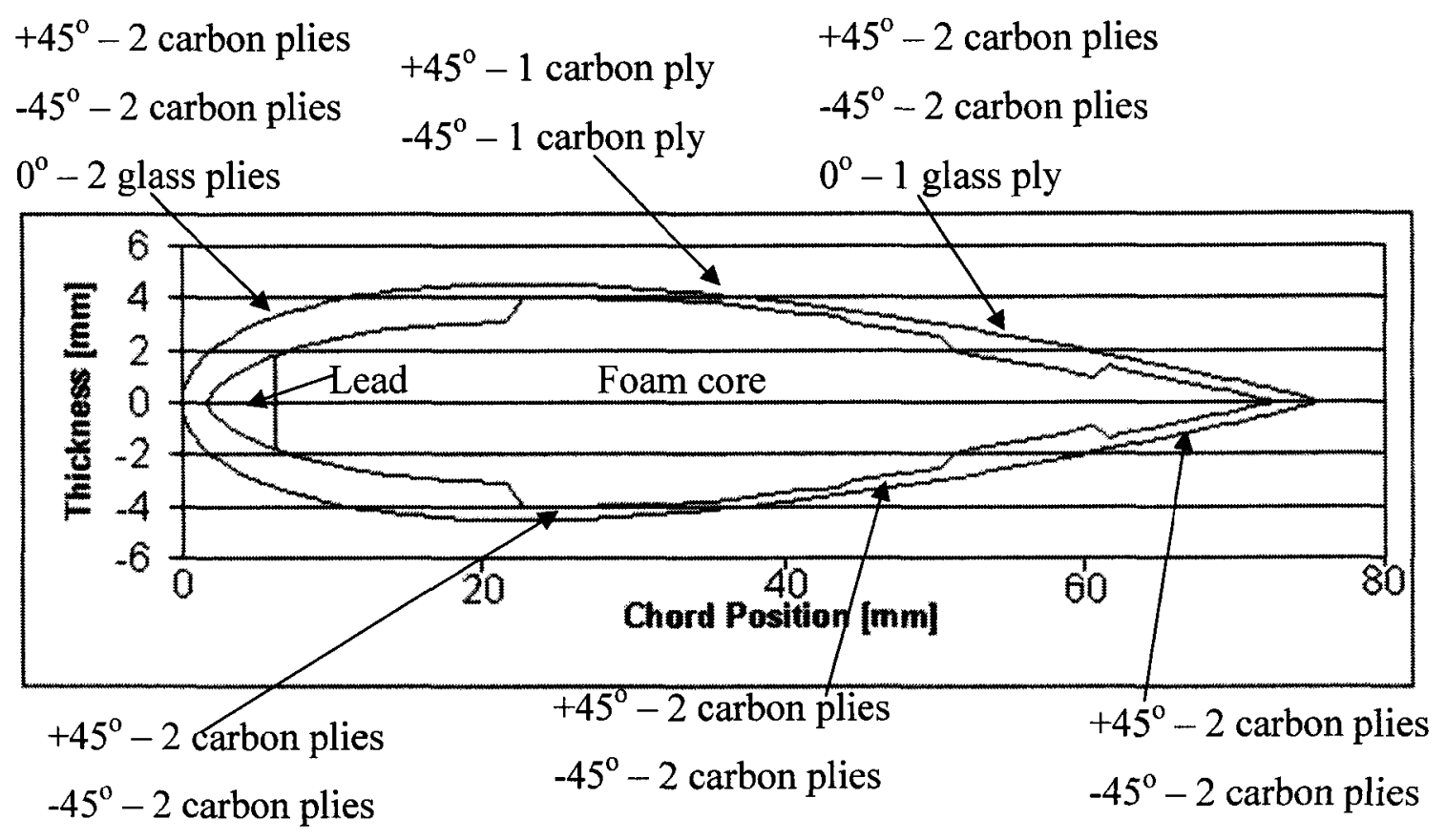

Figure 6.3: Main blade section of the SHARCS rotor blade 
Table 6.2: SHARCS main blade lay-up

\begin{tabular}{cc}
\hline Chord-wise Position [mm] & Lay-up \\
\hline \hline $0-21.6$ & {$[0 / 45 /-45 /-45 / 45 / 0]$} \\
$21.6-30.6$ & {$[45 /-45 /-45 / 45]$} \\
$30.6-44.6$ & {$[45 /-45]$} \\
$44.6-51.4$ & {$[45 /-45 /-45 / 45]$} \\
$51.4-60.7$ & {$[45 /-45 / 0 /-45 / 45]$} \\
$60.7-75.3$ & {$[45 /-45 /-45 / 45]$} \\
\hline
\end{tabular}

The majority of the blade lay-up (81.4\%) was balanced and symmetric laminate, which eliminated the coupling sub-matrix [B] in Eq. (3.2), and ensures that there are no adjacent layers with identical angles. The general rule of thumb that there should not be more than three layers of the same orientation layered on each other, as delamination between the layers will occur. This was imposed for the design. The [45/-45] lay-up was the only anti-symmetric portion of the blade; it would induce no shear deformation due to a direct stress. Thus the blade was a compromise between the benefits of a symmetric and antisymmetric lay-up.

The reason there were two $+45^{\circ}$ and two $-45^{\circ}$ uni-directional plies for most of the profile, but only one of each $45^{\circ}$ ply for a small region was to achieve the desired torsional rigidity for the section. Two $\pm 45^{\circ}$ plies around the entire profile was found to give excessive torsional rigidity; a small region with one $\pm 45^{\circ}$ ply substantially reduced this rigidity without sacrificing significantly the safety factor. It should be noted that 
rounding of the ply edges using resin should be considered in the manufacturing process to decrease the stress concentrations found at the free edges of a laminate.

An extra layer of $0^{\circ}$ glass fibre was required near the trailing edge to increase the lead-lag rigidity. It was found through design iterations that this location minimized the stiffening effect on the flapping rigidity, which was in the required range. While less glass fibre would have been required if the plies were placed directly at the trailing edge, the extra material increased the safety factor.

Furthermore, a foam core was used in the centre of the rotor blade to prevent the skin from buckling or wrinkling. The foam core also transmits the shear to the composite layup. A specific foam material was not selected, as the selected foam would have to be able to withstand the temperatures and pressures experienced during the manufacturing process of the rotor blade. As the manufacturer of the SHARCS rotor blade has yet to provide these details, the specific foam material was not selected at this time.

\subsubsection{Root Lay-up Design}

The root required strengthening to increase the minimum safety factor for the rotor blade. An additional reason for adding layers to the root was the general rule for joining composite structures to have a quasi-isotropic lay-up to avoid generating more complex 
stress fields at the joint than what would already occur in practice. For example, a bolted joint creates considerable stress concentrations, which could lead to various failure modes if the joint is not properly designed. While that was not possible for this design due to the different composite materials and the different ply thicknesses used, it was possible to reduce the magnitude of the variation in the stiffness properties for different orientations to the $\mathrm{x}$-axis. As the joining method has yet to be decided, the strengthening was a precaution, and was not applied to a specific location where the joint would be created. Additional strengthening may be required once the joining method has been determined and the resulting stress field has been calculated.

The root lay-up used the same lay-up as the main blade with one exception. The root was strengthened by adding a $0^{\circ}$ glass fibre ply to the main blade lay-up wherever there was no glass fibre plies. This substantially increased the stiffness values for this region of the rotor blade without increasing its global stiffness appreciably since the root only accounted for $10 \%$ of the blade span. The resulting composite lay-up for the root is given in Table 6.3, and illustrated in Figure 6.4.

Table 6.3: SHARCS root lay-up

\begin{tabular}{cc}
\hline Chord-wise Position $[\mathrm{mm}]$ & Lay-up \\
\hline $0-21.6$ & {$[0 / 45 /-45 /-45 / 45 / 0]$} \\
$21.6-30.6$ & {$[45 /-45 / 0 /-45 / 45]$} \\
$30.6-44.6$ & {$[45 / 0 /-45]$} \\
$44.6-75.3$ & {$[45 /-45 / 0 /-45 / 45]$} \\
\hline
\end{tabular}


As stated previously, the safety factor was increased from 1.18 to 1.9 at the root with the change in the composite ply lay-up.

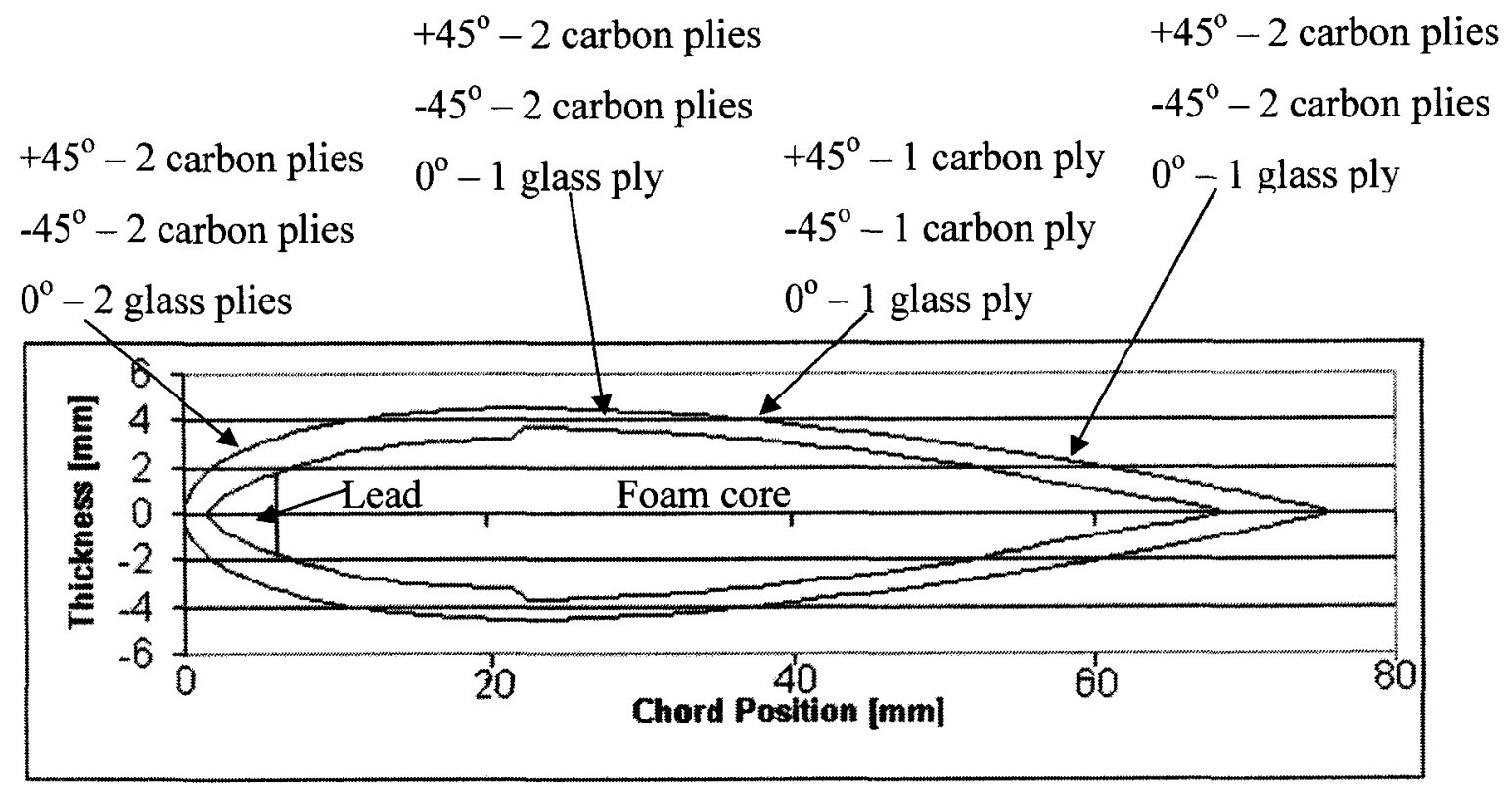

Figure 6.4: Blade root lay-up of the SHARCS rotor blade

\subsubsection{Anhedral Tip Lay-up Design}

The anhedral tip occupies the last $10 \%$ of the rotor blade span. The purpose of the anhedral tip was to move up and down to change the position of the generated tip vortices to reduce BVI noise. Due to the limited actuator power and the high centrifugal forces at the blade tip, the decision was made to make the anhedral tip as light as possible to reduce the required actuator power, as the centrifugal force the actuator must overcome depends on the mass of the tip. 
No attempt was made to match the rigidities of the anhedral tip to those of the main blade. It was decided to make the anhedral tip significantly stiffer than the main blade. As the tip would be stiffer, its natural frequency would be higher then that of the main blade. Due to the fact that the anhedral tip would be many times lighter than the main blade, the higher natural frequency of the tip would not be able to affect the natural frequency of the main blade. By making the anhedral tip very light and stiff, it was initially analyzed as a tip mass as opposed to a part of the structure, which simplified preliminary calculations.

As weight and stiffness was the driving design factor, the anhedral tip was made entirely out of carbon fibre uni-directional pre-preg $\left(0^{\circ},+45^{\circ}\right.$, and $\left.-45^{\circ}\right)$. While there were higher stiffness carbon fibres pre-pregs available, the same carbon fibre pre-preg that was used for the main blade was used for the anhedral tip to keep material cost down.

Figure 6.5 shows the anhedral tip profile, while Table 6.4 provides the lay-up details. It should be noted that there was no lead ballast for the anhedral tip. This was done to keep the weight as low as possible. As a result the centre of mass was not at the $1 / 4$-chord, but at $43.8 \%$ chord. Since the mass was low and the length of the tip was short, it was deemed that no aeroelastic stability problem would arise. The mass of the anhedral tip was found to be $16.5 \mathrm{~g}$. 
Table 6.4: Anhedral tip lay-up

\begin{tabular}{cc}
\hline Chordwise Position $[\mathrm{mm}]$ & Lay-up \\
\hline \hline $0-18.5$ & {$[0 / 45 / 0 /-45 / 0]$} \\
$18.5-75.3$ & {$[45 / 0 /-45]$} \\
\hline
\end{tabular}

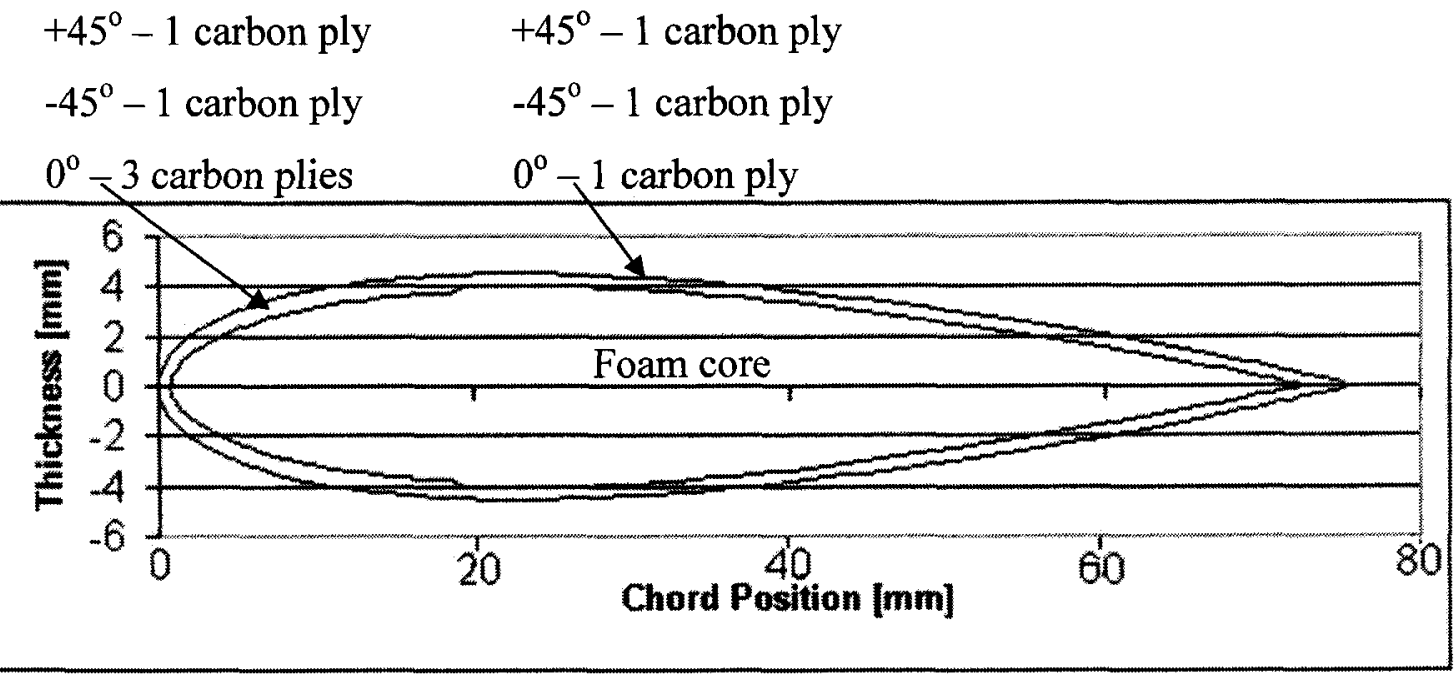

Figure 6.5: Anhedral tip lay-up for the SHARCS rotor blade

\subsubsection{Design Summary}

Table 6.5 provides a summary of the different lay-ups at the different span-wise positions (root, blade, anhedral tip) and the different chord-wise positions. Although no $90^{\circ}$ ply was used, the lead-lag stiffness was at the target value. That meant that no $90^{\circ}$ ply could be used to increase the number of cross-plies, which could have increased the inter-fibre safety factor. The carbon fibre ply thickness were thinner then commonly used for 
composite structures, but were required to obtain the correct rotating natural frequency characteristics. Communications with the carbon fibre manufacturer HEXCEL indicated that the thin ply thickness was fesible.

Table 6.5: Summary of SHARCS rotor blade lay-up summary

\begin{tabular}{ccccc}
\hline Spanwise Position & Chordwise Position & $\begin{array}{c}+\& \text { Fibreglass } \\
0^{\circ} \text { Carbon Fibre } \\
0.5 \text { mm ply } \\
\text { thickness }\end{array}$ & $\begin{array}{c}0.065 \text { mm per } \\
\text { ply }\end{array}$ & $\begin{array}{c}0.065 \text { mm ply } \\
\text { thickness }\end{array}$ \\
\hline \hline Root & $0-21.6$ & 2 & 2 & - \\
& $21.6-30.6$ & 1 & 2 & - \\
& $30.6-44.6$ & 1 & 1 & - \\
& $44.6-75.3$ & 1 & 2 & - \\
\hline Blade & $0-21.6$ & 2 & 2 & - \\
& $21.6-30.6$ & 0 & 2 & - \\
& $30.6-44.6$ & 0 & 1 & - \\
& $44.6-51.4$ & 0 & 2 & - \\
& $51.4-60.7$ & 1 & 2 & 1 \\
\hline Anhedral Tip & $60.7-75.3$ & 0 & 2 & - \\
& $0-18.5$ & - & 1 & 1 \\
\hline
\end{tabular}

This design resulted in the stiffness values shown in Table 6.6. The initial target stiffness estimates were calculated using SMARTROTOR [Nitzsche 2005], a computer program based on the application of natural frequency equations for isotropic properties. The design stiffness values were obtained through iterative design analysis with the commercial finite element method (FEM) software, ANSYS, in which the rotating natural frequencies were compared with the requirements, and the design modified as 
needed. It should be noted that the initial estimate made by SMARTROTOR was fairly accurate, except for the torsional rigidity $G J$ (Table 6.6).

Table 6.6: Design versus target stiffness values

\begin{tabular}{lcccc}
\hline & \multicolumn{2}{c}{ Design [Blade] } & Design [Root] & \multicolumn{2}{c}{ Design [Tip] Target } \\
\hline El flapping $\left[\mathrm{Nm}^{2}\right]$ & 30.8 & 47.3 & 69.4 & 40 \\
El lead-lag $\left[\mathrm{Nm}^{2}\right]$ & 1608 & 2709 & 3403 & 1600 \\
GJ $\left[\mathrm{Nm}^{2}\right]$ & 61.1 & 60.7 & 42.3 & 50 \\
\hline
\end{tabular}

The torsional rigidity target of $50\left[\mathrm{Nm}^{2}\right]$ was near the low end of the rotating natural frequency range, but as a smart spring would lower the rotating natural frequency, the torsional rigidity was increased to put the torsional rotating frequency at the upper half of the required range. Thus when the smart spring is activated, the spring could be tuned so that the torsional rotating frequency would be at the low end of the required range.

\subsection{MSC.Nastran/Patran Finite Element Model Validation}

Before an FEM model of the SHARCS rotor blade could be created to calculate the rotating natural frequencies, validation of the proper use of the finite element analysis program was carried out. To this end, the MSC.Nastran FEA software was used and four general cases were identified for tests on the accuracy of the models. These tests were aimed to verify the ability of the program to handle both isotropic (lead and foam) and composite materials (carbon and fiberglass) for determining the natural frequencies with 
these types of materials. As a learning exercise with the software the problem of an isotropic cantilever beam was first analyzed.

\subsubsection{Isotropic Beam}

In order to validate the MSC.Nastran models developed, an isotropic beam with similar GJ and EI values and mass per unit length of an early iteration of the SHARCS rotor blade was considered. The FEM structured mesh used is shown in Figure 6.6, which contained 463 nodes and 200 elements. The model consisted of isotropic material properties applied to 3-D solid homogeneous elements. Following an iterative process, the resulting isotropic beam properties that would provide the desired stiffness values were obtained and presented in Table 6-7.

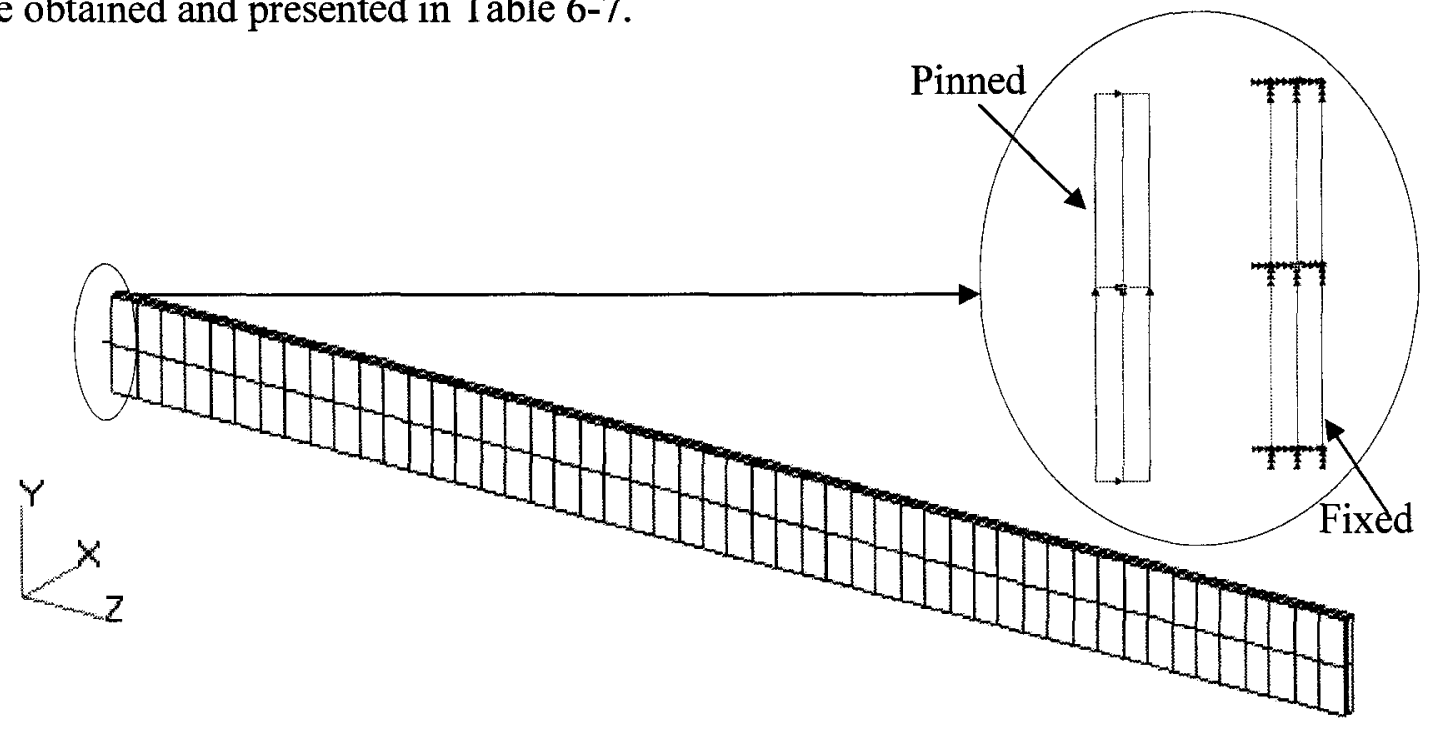

Figure 6.6: MSC.NASTRAN isotropic beam model 
Table 6.7: Isotropic beam properties

\begin{tabular}{|c|c|}
\hline & Isotropic Beam \\
\hline$x[\mathrm{~m}]$ & 0.01 \\
\hline $\mathrm{h}[\mathrm{m}]$ & 0.07066 \\
\hline $\mathrm{E}[\mathrm{Pa}]$ & $6.66 \mathrm{E}+09$ \\
\hline $\mathrm{G}[\mathrm{Pa}]$ & $2.55 E+09$ \\
\hline$l_{x x}\left[m^{4}\right]$ & 5.89E-09 \\
\hline $\operatorname{lYY}\left[\mathrm{m}^{4}\right]$ & 2.94E-07 \\
\hline$J\left[\mathrm{~m}^{4}\right]$ & 2.14E-08 \\
\hline$E_{x x}\left[\mathrm{Nm}^{2}\right]$ & 39.2 \\
\hline$E I_{Y Y}\left[\mathrm{Nm}^{2}\right]$ & 1957.19 \\
\hline $\mathrm{GJ}\left[\mathrm{Nm}^{2}\right]$ & 54.6 \\
\hline
\end{tabular}

\subsubsection{Static Deflection of a Cantilevered Isotropic Beam}

To verify the accuracy of the MSC.Patran isotropic beam model, a $20 \mathrm{~N}$ load was applied at the tip of the structure and the analysis was carried out with MSC.Nastran. The computed static deflections at the tip of the cantilever were compared with the theoretical solution, which is given by:

$$
\delta=\frac{W \cdot L^{3}}{3 E I}
$$


where: $\delta$ was the displacement of the tip of the beam; $W$ the load applied; $L$ the length of the beam; and $E I$ the flexural rigidity of the beam. Table 6.8 shows the values obtained from MSC.Nastran compared with the theoretical values.

Table 6.8: MSC.Nastran and theoretical static deflection results

\begin{tabular}{lccc}
\hline Deflection & Theoretical & Nastran & Difference [\%] \\
\hline \hline Flap [m] & 0.17 & 0.168 & 1.18 \\
Lead-Lag [m] & 0.0034 & 0.0034 & 0 \\
\hline
\end{tabular}

To compare the torsional rigidity, $G J$, of the beam, a $2 \mathrm{Nm}$ torque moment, $M$, was applied. The theoretical equation for the angle of twist, $\theta$, due to the torque is [Timoshenko and MacCullough 1940]:

$$
\theta=\frac{M \cdot L}{G J}
$$

with: $\quad J=\beta h \eta^{3}$ for a Timoshenko rectangular beam

Since MSC.Nastran only calculates deflections, the angle of twist was obtained from the deflection components and is shown in Table 6.9 together with the theoretical value.

Table 6.9: MSC.Nastran and theoretical static torsion results

\begin{tabular}{llll}
\hline Twist & Theoretical & Nastran & Difference [\%] \\
\hline \hline Torsion [rad] & 0.0366 & 0.0335 & 8.63 \\
\hline
\end{tabular}


There was a discrepancy of approximately $8.6 \%$ in this result, which may be attributed to the warping caused by the twisting of the rectangular section beam, which was not considered in the deflection components of the FEM results.

\subsubsection{Non-Rotating Natural Frequencies of an Isotropic Beam}

The next step for validating the model was to compare the natural frequencies of the isotropic beam to those predicted by theory. This was done for two different boundary conditions, namely, hingeless (fixed-free) and articulated [pinned (flap, lead-lag) fixed (torsion)-free]. The latter case would resemble the actual operating condition of the articulated rotor blade, using lead-lag and flapping hinges, and a stiff pitch link assembly (which imposes a constraint on the twisting motion at the root).

For the theoretical results, Eq. (2.6a) and (2.6b) were used. Table 6.10 shows the comparison between the MSC.Nastran and theoretical results, the largest deviation was in the torsion mode, where there was a $5 \%$ discrepancy. 
Table 6.10: MSC.Nastran and theoretical natural frequencies of the non-rotating isotropic beam

\begin{tabular}{lcccc}
\hline & $1^{\text {st }}$ Flap & $2^{\text {nd }}$ Flap & $1^{\text {st }}$ Lead-Lag & $1^{\text {st }}$ Torsion \\
Hingeless & & & & \\
\hline Theoretical [Hz] & 4.790 & 29.97 & 33.82 & 122.5 \\
Nastran [Hz] & 4.817 & 30.20 & 33.80 & 129.31 \\
Difference [\%] & 0.57 & 0.78 & 0.06 & 5.56 \\
& & & & \\
Articulated & & & & \\
\hline Theoretical [Hz] & 21.006 & 68.07 & 148.33 & 122.5 \\
Nastran $[\mathrm{Hz}]$ & 21.006 & 68.19 & 146.65 & 127.08 \\
Difference [\%] & 0.00 & 0.18 & 1.13 & 3.74 \\
\hline
\end{tabular}

Figures 6.7 to 6.9 show the frequency response diagrams to a random unit load input applied to the tip of the rotor blade with articulated boundary conditions. Using these graphs, it was possible to obtain the necessary information about the natural frequencies of the articulated blade design.

Also from the plots, it is noticed that both the flapping and lead-lag degrees of freedom show high displacement amplitude dynamic response in the low frequency range. This is to be expected due to the rigid body mode that is present with the pinned boundary conditions. 


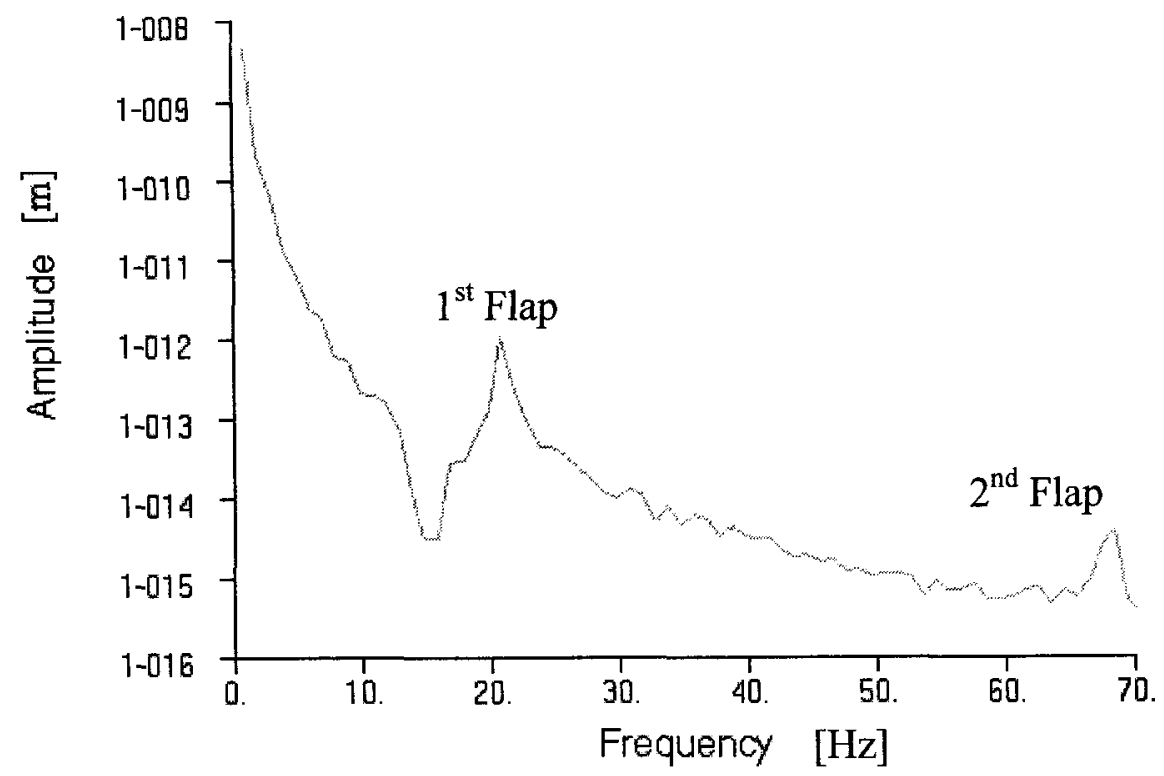

Figure 6.7: Flap frequency response diagram for articulated blade to random unit input applied to the rotor blade tip

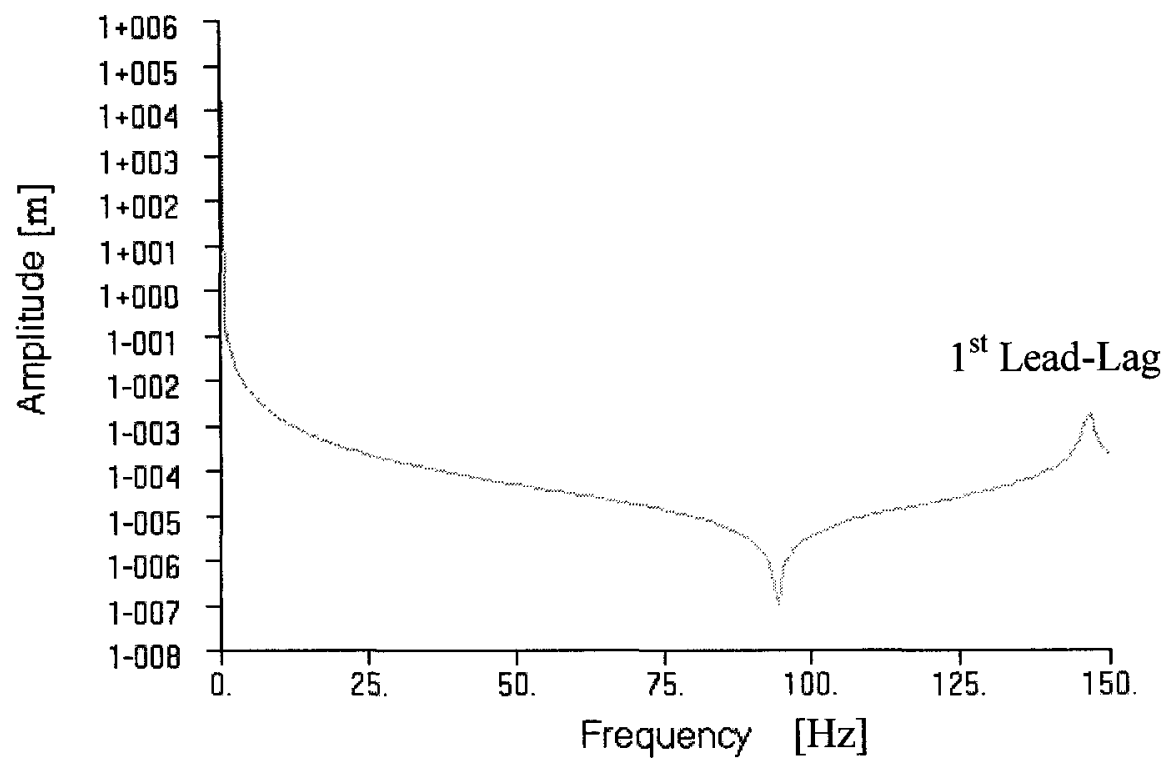

Figure 6.8: Lead-lag frequency response diagram for articulated blade to random unit input applied to the rotor blade tip 


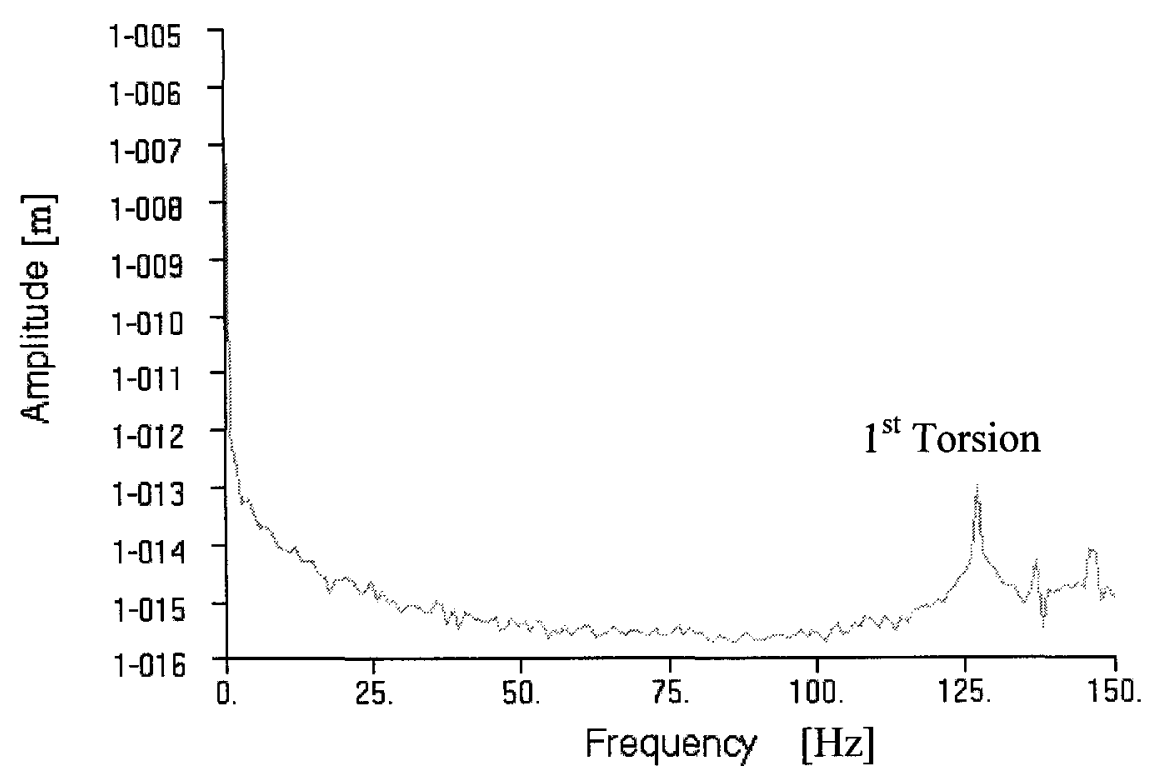

Figure 6.9: Torsional frequency response diagram for articulated blade to random unit input applied to the rotor blade tip

\subsubsection{Composite Beam Static Deflection}

The MSC.Nastran/Patran composite rotor blade model was next subjected to the same load characteristics of the isotropic beam and the static deflections were calculated. Both models had similar $E I, G J$ and masses per unit length. The composite blade model was composed of isotropic 3-D homogeneous solid elements, orthotropic 2-D laminated shell elements and orthotropic 3-D solid homogeneous elements.

As with the isotropic beam analysis, the boundary conditions were pinned and fixed at one end, and free at the other. Table 6.11 shows the results of this analysis. As expected, 
the deflections obtained for the two cases are significantly different due to the anisotropy of the composite beam.

Table 6.11: Composite rotor blade static deflection comparison

\begin{tabular}{lccc}
\hline Deflection & Isotropic & $\begin{array}{c}\text { Composite } \\
\text { (Nastran) }\end{array}$ & Difference [\%] \\
\hline \hline Flap [m] & 0.17 & 0.126 & 25.88 \\
Lead-Lag [m] & 0.0034 & 0.00294 & 13.53 \\
Torsion [rad] & 0.0335 & 0.0252 & 24.78 \\
\hline
\end{tabular}

\subsubsection{Composite Beam Natural Frequencies}

To validate the natural frequencies of a composite blade as calculated by MSC.Nastran, the composite beams used in Abramovich et al (1995) and Abdelnaser and Singh (1992) were modeled and the natural frequencies were calculated. The MSC.Nastran structured mesh is shown in Figure 6.10 and contains 1275 nodes and 800 elements. 3-D orthotropic homogeneous solid elements were used to produce the composite beam model. 


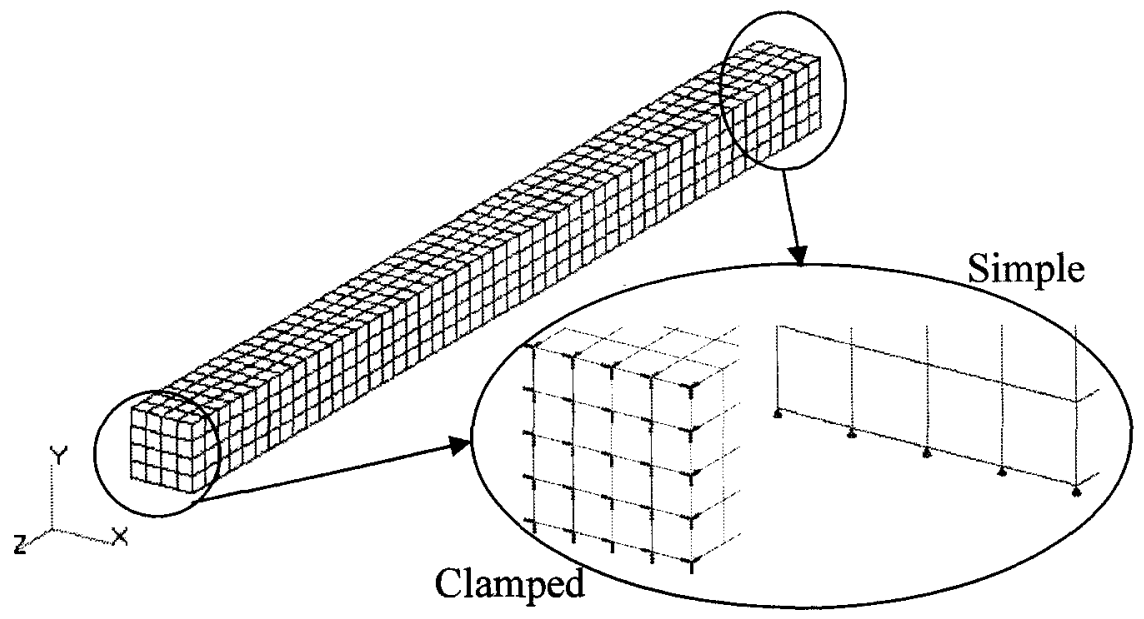

Figure 6.10: MSC.Nastran composite beam model

In these references, a beam with a lay-up of $\left[0^{\circ} / 90^{\circ} / 90^{\circ} / 0^{\circ}\right]$ made from graphite/epoxy (AS/3501-6) with the aspect ratio $L / h=15$ resulting in the following lamina properties: $E_{1}=144.8 \mathrm{GPa}, E_{2}=9.65 \mathrm{GPa}, G_{12}=G_{13}=4.14 \mathrm{GPa}, G_{23}=3.45 \mathrm{GPa}, \rho=1389.23$ $\mathrm{kg} / \mathrm{m}^{3}$, and $v_{12}=0.3$ was analyzed. The values shown in the following tables are the nondimensional frequencies, $\omega_{\text {Non-Dim }}$, obtained using the following normalization:

$$
\omega_{\text {Non-Dim }}=\omega L^{2} \sqrt{\frac{\rho}{E_{1} h^{2}}}
$$

As can be seen in Table 6.12, the present FEM results compare well with those presented in Chandrasekhara et al (1990), Abramovich et al (1995) and Kiral and Yildirim (2000), validating the modeling process that was employed. 
Table 6.12: Non-dimensional frequency comparison for $\left[0^{\circ} / 90^{\circ} / 90^{\circ} / 0^{\circ}\right]$ graphite/epoxy

$$
\text { beam with } L / h=15
$$

\begin{tabular}{|c|c|c|c|c|}
\hline & Clamped-Free & & & \\
\hline & $\begin{array}{l}\text { Chandrasekhara } \\
\text { et al (1990) }\end{array}$ & $\begin{array}{l}\text { Abramovich } \\
\text { et al (1995) }\end{array}$ & $\begin{array}{l}\text { Kiral and } \\
\text { Yildirim (2000) }\end{array}$ & Nastran \\
\hline$\overline{\text { Mode } 1}$ & 0.9241 & 0.9241 & 0.9215 & 0.9240 \\
\hline Mode 2 & 4.8925 & 4.8925 & 4.8832 & 4.9357 \\
\hline \multirow[t]{3}{*}{ Mode 3} & 11.4400 & 11.4401 & 11.4251 & 11.6323 \\
\hline & \multicolumn{4}{|l|}{ Clamped-Clamped } \\
\hline & $\begin{array}{l}\text { Chandrasekhara } \\
\text { et al (1990) }\end{array}$ & $\begin{array}{l}\text { Abramovich } \\
\text { et al (1995) }\end{array}$ & $\begin{array}{l}\text { Kiral and } \\
\text { Yildirim (2000) }\end{array}$ & Nastran \\
\hline$\overline{\text { Mode } 1}$ & 4.5940 & 4.5941 & 4.5869 & 4.6521 \\
\hline Mode 2 & 10.2906 & 10.2908 & 10.2810 & 10.5151 \\
\hline \multirow[t]{3}{*}{ Mode 3} & 16.9659 & 16.9662 & 16.9551 & 17.4476 \\
\hline & Clamped-Simple & & & \\
\hline & $\begin{array}{l}\text { Chandrasekhara } \\
\text { et al (1990) }\end{array}$ & $\begin{array}{l}\text { Abramovich } \\
\text { et al (1995) }\end{array}$ & $\begin{array}{l}\text { Kiral and } \\
\text { Yildirim (2000) }\end{array}$ & Nastran \\
\hline Mode 1 & 3.5254 & 3.5254 & 3.5183 & 3.5509 \\
\hline Mode 2 & 9.4423 & 9.4424 & 9.4299 & 9.5920 \\
\hline \multirow[t]{3}{*}{ Mode 3} & 16.3839 & 16.3841 & 16.3700 & 16.7635 \\
\hline & Simple-Simple & & & \\
\hline & $\begin{array}{l}\text { Chandrasekhara } \\
\text { et al (1990) }\end{array}$ & $\begin{array}{l}\text { Abramovich } \\
\text { et al (1995) }\end{array}$ & $\begin{array}{l}\text { Kiral and } \\
\text { Yildirim (2000) }\end{array}$ & Nastran \\
\hline$\overline{\text { Mode } 1}$ & 2.5023 & 2.5024 & 2.4959 & 2.5062 \\
\hline Mode 2 & 8.4812 & 8.4813 & 8.4663 & 8.5603 \\
\hline Mode 3 & 15.7558 & 15.7559 & 15.7384 & 16.0296 \\
\hline
\end{tabular}


Table 6.13 presents the natural frequencies of an $\left[0^{\circ} / 90^{\circ} / 90^{\circ} / 0^{\circ}\right]$ AS/3501-6 graphite epoxy beam with the same properties as the beam used for the preparation of Table 6.11 , but with $\rho=1000 \mathrm{~kg} / \mathrm{m}^{3}$, and $L / h=10$.

Table 6.13: Non-dimensional frequency comparison for $\left[0^{\circ} / 90^{\circ} / 90^{\circ} / 0^{\circ}\right]$ graphite/epoxy beam with $L / h=10$

\begin{tabular}{|c|c|c|c|}
\hline & Fixed-Fixed & & \\
\hline & $\begin{array}{l}\text { Abdelnaser and } \\
\text { Singh (1992) }\end{array}$ & $\begin{array}{l}\text { Abramovich and } \\
\text { Livshits (1994) }\end{array}$ & Nastran \\
\hline Mode 1 & 3.7751 & 3.7576 & 3.7629 \\
\hline Mode 2 & 8.0440 & 7.8718 & 7.9589 \\
\hline \multirow[t]{3}{*}{ Mode 3} & 12.9980 & 12.5730 & 12.8183 \\
\hline & Fixed-free & & \\
\hline & $\begin{array}{l}\text { Abdelnaser and } \\
\text { Singh (1992) }\end{array}$ & $\begin{array}{l}\text { Abramovich and } \\
\text { Livshits (1994) }\end{array}$ & Nastran \\
\hline Mode 1 & 0.8891 & 0.8818 & 0.8885 \\
\hline Mode 2 & 4.1790 & 4.0259 & 4.1711 \\
\hline \multirow[t]{3}{*}{ Mode 3} & 9.1916 & 9.1084 & 9.1647 \\
\hline & Hinged-Hinged & & \\
\hline & $\begin{array}{l}\text { Abdelnaser and } \\
\text { Singh (1992) }\end{array}$ & $\begin{array}{l}\text { Abramovich and } \\
\text { Livshits (1994) }\end{array}$ & Nastran \\
\hline$\overline{\text { Mode } 1}$ & 2.3188 & 2.3194 & 2.3078 \\
\hline Mode 2 & 7.0170 & 7.0024 & 6.9309 \\
\hline Mode 3 & 12.1316 & 12.0367 & 11.8598 \\
\hline
\end{tabular}

The results shown in Tables 6.12 and 6.13 provide convincing evidence of the accuracy of FEM composite beam models developed in MSC.Nastran for the natural frequencies of non-rotating blade cases. 


\subsection{ANSYS Validation}

As MSC.Nastran does not possess the ability to calculate the rotational natural frequencies, an alternative FEM package, ANSYS, was therefore used for these cases. As MSC.Nastran was already validated, ANSYS was validated in comparison to MSC.Nastran for the non-rotating cases. Table 6.14 shows the comparison of the FEA models made with the two programs for an early iteration SHARCS rotor blade.

Table 6.14: ANSYS and MSC.Nastran model comparison

\begin{tabular}{l|cccc}
\hline Hingeless & 1st Flap & 2nd Flap & 1st Lead Lag & 1st Torsion \\
\hline Nastran [Hz] & 5.59 & 34.87 & 36.5 & 150.0 \\
ANSYS [Hz] & 5.50 & 34.33 & 35.18 & 150.18 \\
Difference [\%] & 1.55 & 1.56 & 3.61 & 0.12 \\
\hline
\end{tabular}

\begin{tabular}{l|cccc}
\hline Articulated & 1st Flap & 2nd Flap & 1st Lead Lag & 1st Torsion \\
\hline Nastran [Hz] & 24.39 & 78.8 & 157.22 & 145.62 \\
ANSYS [Hz] & 24.16 & 77.72 & 150.73 & 143.82 \\
Difference [\%] & 0.96 & 1.38 & 4.13 & 1.24 \\
\hline
\end{tabular}

It can be seen that the ANSYS finite element program compares well with the validated MSC.Nastran. 


\subsection{Composite Rotor Blade Natural Frequencies}

The final step in the present design was to create a model of the composite rotor blade in ANSYS and perform a natural frequency analysis for the rotating beam (fan plots). The thickness of the rotor blade composite skin was sufficiently thin to be accurately modeled as a shell. Therefore the model was created using linear layered composite shell elements (SHELL99) and 20 noded solid elements (SOLID95). SHELL99 elements were selected, as they allow up to 250 composite layers, and the computational time increases at a lower rate with increasing layers than the non-linear equivalent element (SHELL91), shown in Figure 6.11. This allowed the flexibility for changing the layers during design iterations. The FEM mesh that was employed is shown in Figure 6.12, which consisted of 36000 elements and 96500 nodes.

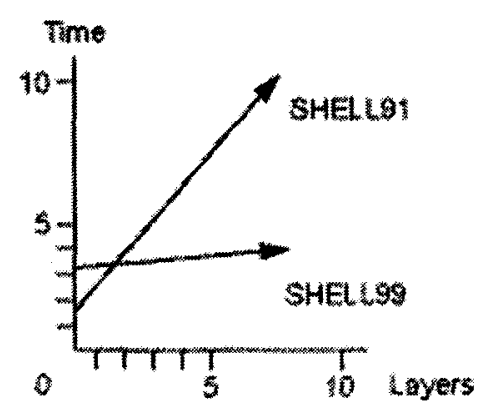

Figure 6.11: Computational time versus number of layers for ANSYS composite shell elements [ANSYS help file, 2005] 


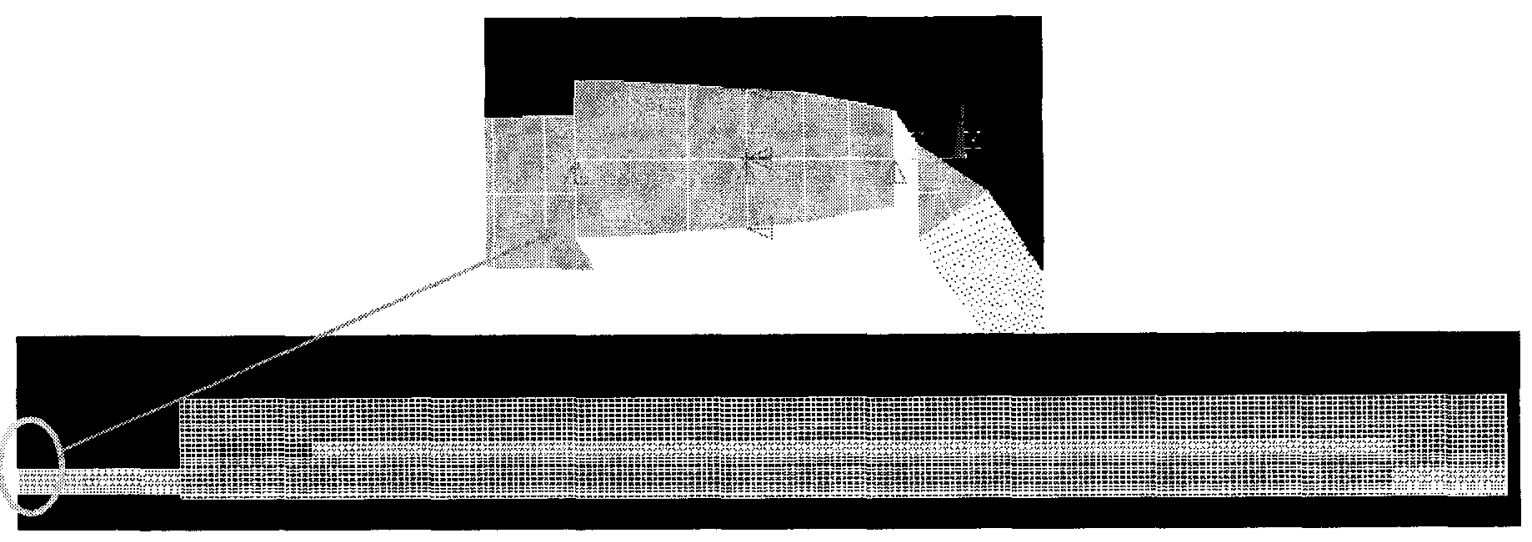

Figure 6.12: ANSYS model of the SHARCS rotor blade

With the ANSYS FEA program validated and the SHARCS rotor blade modeled, the rotating natural frequencies were solved at different rotational speeds. Due to the centrifugal stress caused by the rotational speed, the natural frequencies increased as expected, as the rotational speed was increased. The results are shown in Table 6.15 and in the SHARCS fanplot shown in Figure 6.13.

From the fanplot, it is noted that the 6 per-rev line is close to the rotating natural torsional frequency. As the per-rev lines indicate excitation frequencies, if the hub rotation velocity is decreased by $5 \mathrm{rad} / \mathrm{s}$, the torsional frequency could be excited and could lead to structural failure. It can also be seen from the fanplot that none of the plotted natural frequencies are coupled with another natural frequency. Of particular concern is the coupling of the torsion and flapping mode as that indicates an aeroelastic instability. 
Table 6.15: Articulated rotating natural frequencies for SHARCS rotor blade

\begin{tabular}{|c|c|c|c|c|}
\hline Rotational Speed [rad/s] & $1^{\text {st }}$ Flap $[\mathrm{Hz}]$ & $2^{\text {nd }}$ Flap $[\mathrm{Hz}]$ & $1^{\text {st }}$ Lead-Lag $[\mathrm{Hz}]$ & $1^{\text {st }}$ Torsion $[\mathrm{Hz}]$ \\
\hline 0 & 17.8 & 57.7 & 110.5 & 130.9 \\
\hline 50 & 26.7 & 66.2 & 112.5 & 132 \\
\hline 100 & 43.2 & 85.8 & 118.3 & 133.7 \\
\hline 150 & 61 & 108.1 & 127.3 & 138.8 \\
\hline
\end{tabular}

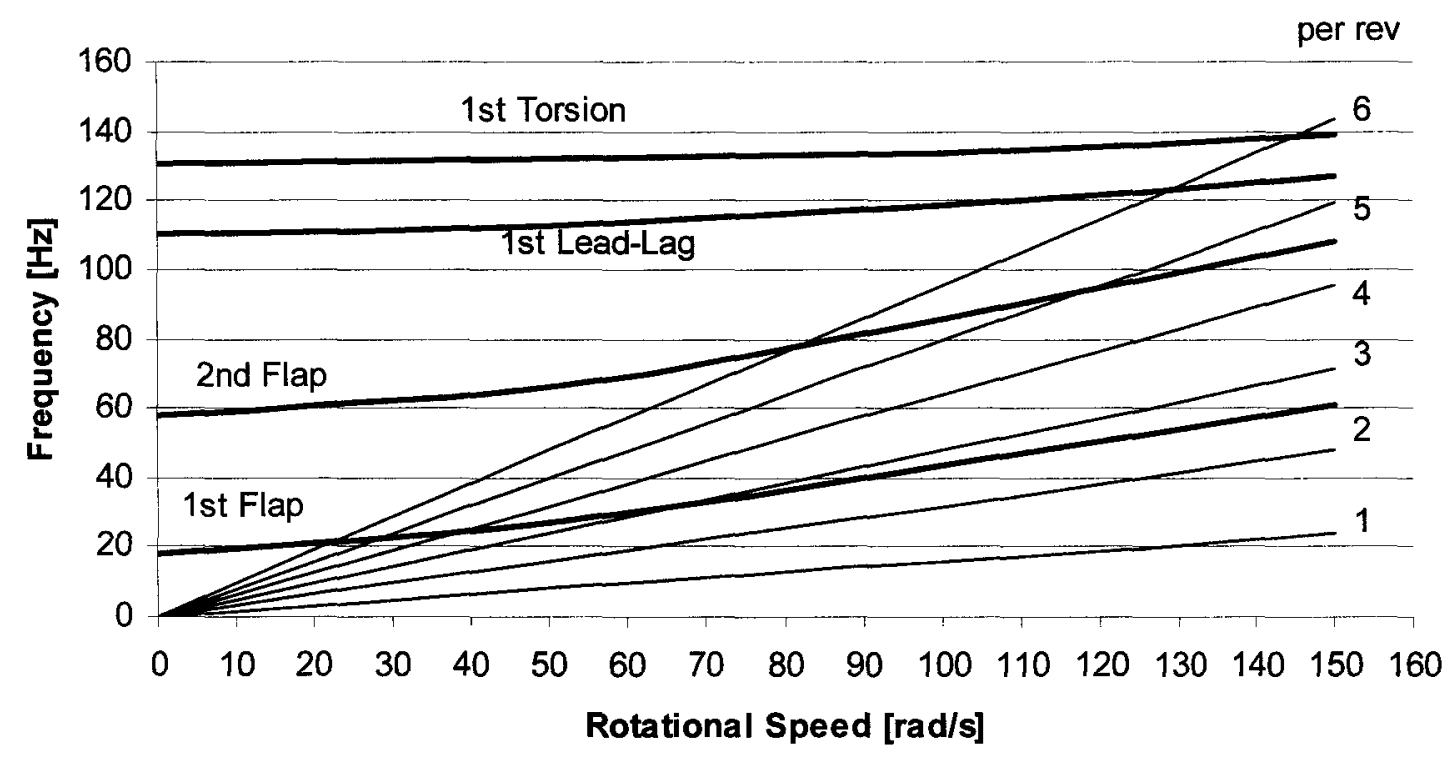

Figure 6.13: SHARCS rotor blade fanplot

From Table 6.16, it can be seen that the rotating frequencies of the SHARCS rotor blade occur in the required ranges, thus meeting that design requirement. The mode shapes of the natural frequencies are shown in Figures $6.14-6.17$ for the SHARCS rotor blade. 
Table 6.16: SHARCS rotating natural frequencies and required frequency range

\begin{tabular}{ccc}
\hline Articulated [per rev] & $\begin{array}{c}\text { SHARCS } \\
\text { at } 150 \mathrm{rad} / \mathrm{s}\end{array}$ & Design Requirement \\
\hline $1^{\text {st }}$ Flap & 2.55 & $2.5-2.8$ \\
$2^{\text {nd }}$ Flap & 4.53 & $4.2-4.7$ \\
$1^{\text {st }}$ Lead-Lag & 5.33 & $4.5-5.5$ \\
$1^{\text {st }}$ Torsion & 5.82 & $5.5-6$ \\
\hline
\end{tabular}

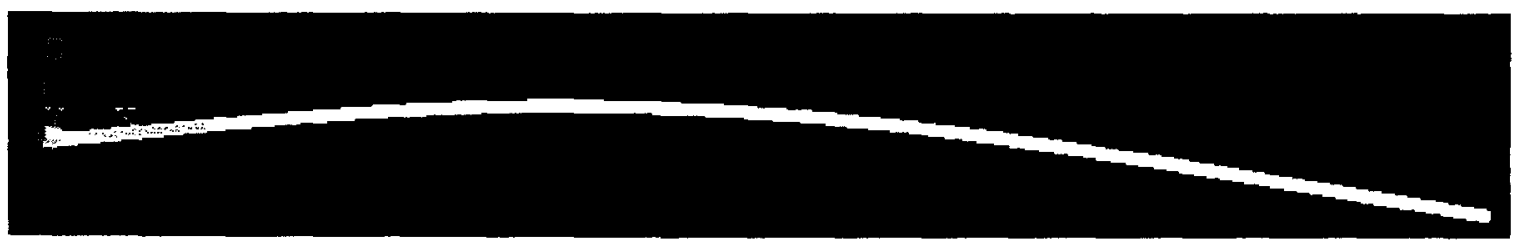

Figure 6.14: SHARCS $1^{\text {st }}$ elastic flapping mode

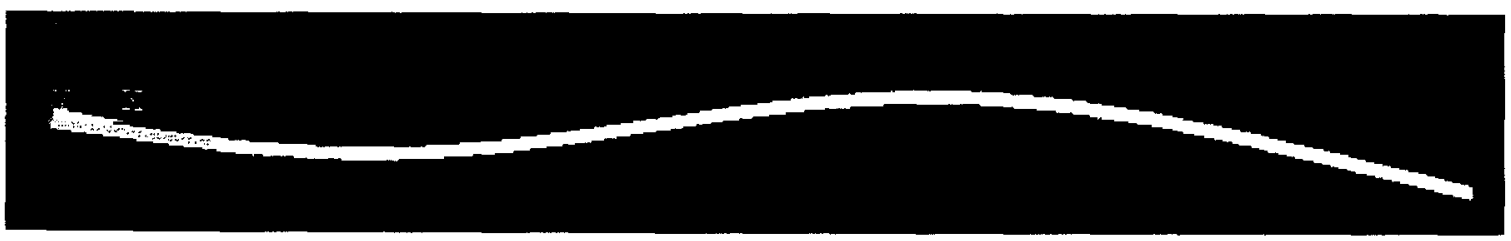

Figure 6.15: SHARCS $2^{\text {nd }}$ elastic flapping mode

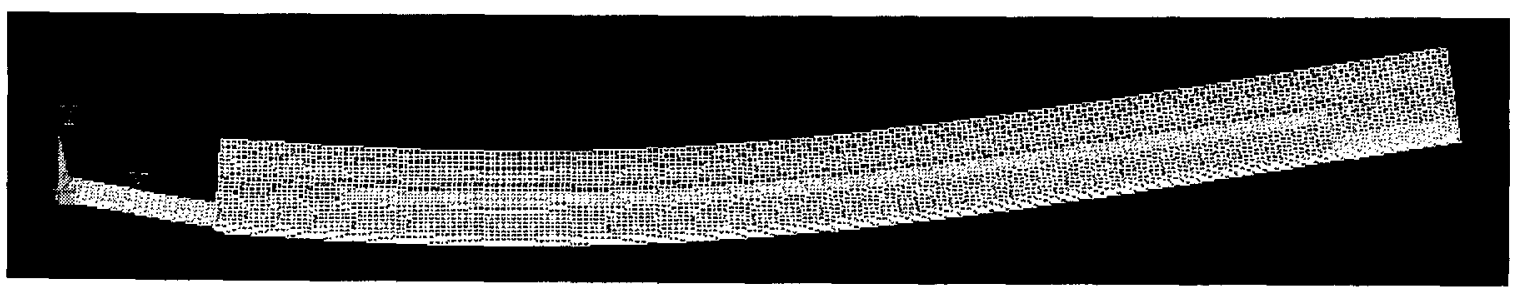

Figure 6.16: SHARCS $1^{\text {st }}$ elastic lead-lag mode 


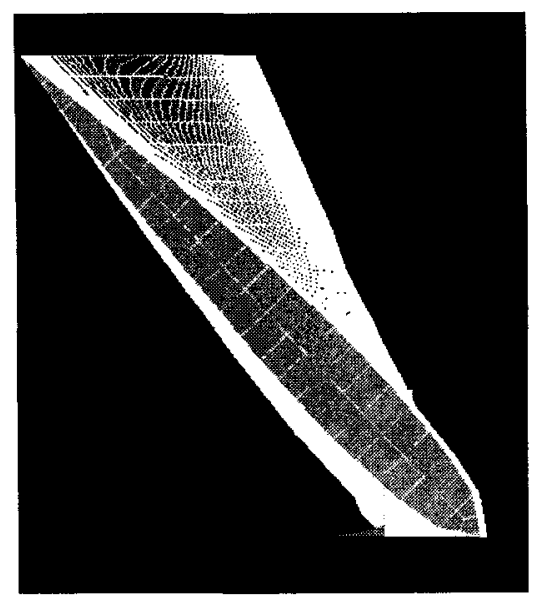

Figure 6.17: SHARCS $1^{\text {st }}$ torsion mode

\subsection{Concluding Remarks}

The preliminary design of the SHARCS rotor blade has been shown. Three sections make up the rotor blade: the root, the main blade, and the anhedral tip. A compromise between anti-symmetric and symmetric laminates was made for the design. The SHARCS rotor blade was modeled and analyzed in the validated FEM software, which provided the rotational natural frequencies of the rotor blade. With the SHARCS rotor blade meeting the required rotating natural frequencies, and having a minimum safety factor of 1.23 , the preliminary design was completed. 


\section{CHAPTER 7 CONCLUSIONS}

A preliminary design of a rotor blade capable of testing the noise and vibration subsystems required for the Smart Hybrid Active Rotor Control System (SHARCS) has been successfully accomplished in this thesis. The rotor blade was scaled to a Lock number of 3.7 and a tip Mach velocity of 0.5 . To achieve this tip Mach velocity, a rotational speed of $150 \mathrm{rad} / \mathrm{s}(1433 \mathrm{rpm})$ was required, which produced the maximum load on the rotor blade of $7500 \mathrm{~N}$. The in-house computer program, SMARTROTOR, was used to provide the initial estimates of the geometric parameters for the required flexural and torsional rigidities. While it was found that good initial estimates for the flexural rigidities were obtained, this was not so for the torsional rigidity. This could be attributed to the 1-D isotropic beam theory assumptions in the SMARTROTOR computer program.

Using the computer program developed at DLR in Germany, the preliminary design of the SHARCS rotor blade was created. The proposed SHARCS rotor blade design has a C-spar, a skin that was fabricated with high strength IM6/epoxy carbon fibre and Sglass/epoxy fibreglass composite and a foam core. Strengthening of the root was 113 
required to obtain a higher safety factor as well as enabling a closer to isotropic properties for attachment to the hub. The anhedral tip, on the other hand, was made lighter and stiffened for reduced actuation forces. An allocation of $136 \mathrm{~g}$ was made for the actuation and sub-systems, which represents $25 \%$ of the rotor blade mass. A safety factor of 1.23 was obtained for the SHARCS rotor blade based on the preliminary structural analysis and laminate theory calculations. These preliminary calculations have employed the "strength of materials" approach with simplifying, but conservative, assumptions. Thus, an overly conservative design of the blade may have been developed which could be more than was indicated by the safety factor. A more detailed stress analysis is required; this is, however, outside the scope of the present study. With the final composite rotor blade design, a finite element analysis using the commercial software ANSYS has also been carried out to determine that the fanplot of the SHARCS blade corresponds to the design requirements. 


\section{REFERENCES}

Abbott, I., Doenhoff, A., Theory of Wing Sections, New York, USA, Dover, 1960.

Abdelnaser A. S., Singh M. P., "Random Response of Symmetric Cross-Ply Composite Beams with Arbitrary Boundary Conditions," American Institute of Aeronautics and Astronautics Journal, 30, 1992.

Abramovich H., Eisenberger M., Shulepov O., "Dynamic Stiffness Analysis of Laminated Beams Using a First-Order Shear Deformation Theory," Composite Structures, 31, pp. 265-271, 1995.

Abramovich H., Livshits A., "Free Vibrations of Non-Symmetric Cross-Ply Laminated Composite Beams," Journal of Sound and Vibration, 176, pp. 597-612, 1994.

Anonymous, Internal Communication, Institute for Flight Mechanics, German Aerospace Research Centre, Braunschweig, Germany, 1993.

ANSYS, Help File, 2005.

Baker, A., Hoskin, B., Composite Materials for Aircraft Structures, New York, USA, American Institute of Aeronautics and Astronautics, 1986.

Bernhard A., Chopra I., "Hover test of a Mach-scaled rotor model with active blade tips," Journal of the American Helicopter Society, 47, pp. 273-284, 2002.

Bernhard A., Chopra I., "Hover Testing of Active Rotor Blade-Tips Using a PiezoInduced Bending-Torsion Coupled Beam," Journal of intelligent material systems and structures, 9, pp. 963-974, 1998. 
Chandrasekhara K., Krisnamurthy K., Roy S., "Free Vibration of Composite Beams Including Rotary Inertia and Shear Deformation," Composite Structures, 14, pp. 269-279, 1990.

Chopra, I., Koratkar, N., "Wind Tunnel Testing of a Smart Rotor Model with TrailingEdge Flaps,” Journal of the American Helicopter Society, 47, pp. 263-272, 2002.

Chopra, I., Koratkar, N., "Open-Loop Hover and Wind Tunnel Testing of Mach-Scaled Rotor with Trailing-Edge Flaps," American Institute of Aeronautics and Astronautics, pp. 1317-1331, 2001.

Chopra, I., Shen, J., "A Parametric Design Study for a Swashplateless Helicopter Rotor with Trailing-Edge Flaps," Journal of the American Helicopter Society, pp. 43-53, January 2004.

Chopra, I., Shen, J., "Aeroelastic Stability of Trailing-Edge Flap Helicopter Rotors," Journal of the American Helicopter Society, 48, pp. 236-243, 2003.

Daniel I., Engineering Mechanics Of Composite Materials, New York, USA, Oxford University Press, 1994.

Enenkl, B., Kloeppel, V., "Rotor Blade Control by Active Helicopter Servo Flaps," in International Forum on Aeroelasticity and Structural Dynamics, 2005.

Inman D., Engineering Vibration, $2^{\text {nd }}$ Edition, Prentice-Hall, 2001.

Kaw, A., Mechanics of Composite Materials, New York, USA, CRC Press, 1997.

Kiral E., Yildirim V., "Investigation of the Rotary Inertia and Shear Deformation Effects on the Out-of-Plane Bending and Torsional Natural Frequencies of Laminated Beams," Composite Structures, 49, pp. 313-320, 2000.

Kollar, L., Springer, G., Mechanics of Composite Structures, New York, USA, Cambridge University Press, 2003 
Leishman, G., Principles of Helicopter Aerodynamics, New York, USA, Cambridge University Press, 2000.

Möller, C., "Strukturelle Auslegung eines Modellrotorblattes mit aktiver Verwindung," Deutschen Zentrums für Luft- und Raumfahrt, Germany, Tech. Rep. Matrikel Nr. $2501172,2004$.

Nitzsche, F. Opoku, D. G., "Acoustic Validation of a New Code Using Particle Wake Aerodynamics and Geometrically Exact Beam Structural Dynamics," The Aeronautical Journal of the UK Royal Aeronautical Society, Vol. 109, No. 1096, pp. 257-267, 2005.

Peters, D., Ko, T., Korn, A., and Rossow, M., "Design of Helicopter Rotor Blades for Desired Placement of Natural Frequencies," Proceedings of the $39^{\text {th }}$ Annual Forum of the American Helicopter Society, Anaheim, CA, pp. 674-689, May 1983.

Puck, A., "Festigkeitsanalyse von Faser-Matrix-Laminaten: Modelle für die Praxis", Carl Hanser Verlag 1996, ISBN 3-446-18194-6.

Puck, A., Kopp, J., Knops, M., "Guidelines for the Determination of the Parameters in Puck's Action Plane Strength Criterion", Composites Science and Technology, 62, pp. 371-378, 2002.

Puck, A., Schurmann, H., "Failure Analysis of FRP Laminates by Means of Physically Based Phenomenological Models", Composites Science and Technology, 62, pp. 16331662, 2002.

Puck, A., Schurmann, H., "Failure Analysis of FRP Laminates by Means of Physically Based Phenomenological Models", Composites Science and Technology, 58, pp. 10451067, 1998.

Raymer, D., Aircraft Design: A Conceptual Approach, Washington, USA, American Institute of Aeronautics and Astronautics, 1999.

de Silva, C., Vibration Fundamental and Practice, Boca Raton, USA, CRC Press, 2000. 
Timoshenko, S., $\mathrm{M}^{\mathrm{ac}}$ Cullough, G., Elements of Strength of Materials, New York, USA, D. Van Nostrand Company, 1940.

Tsai, Composites Design, $4^{\text {th }}$ edition, Think Composites, 1988.

Wierach P., Personal Communication, Carleton University, 2004. 


\section{Appendix A: Iterative Design Process Flow Chart}

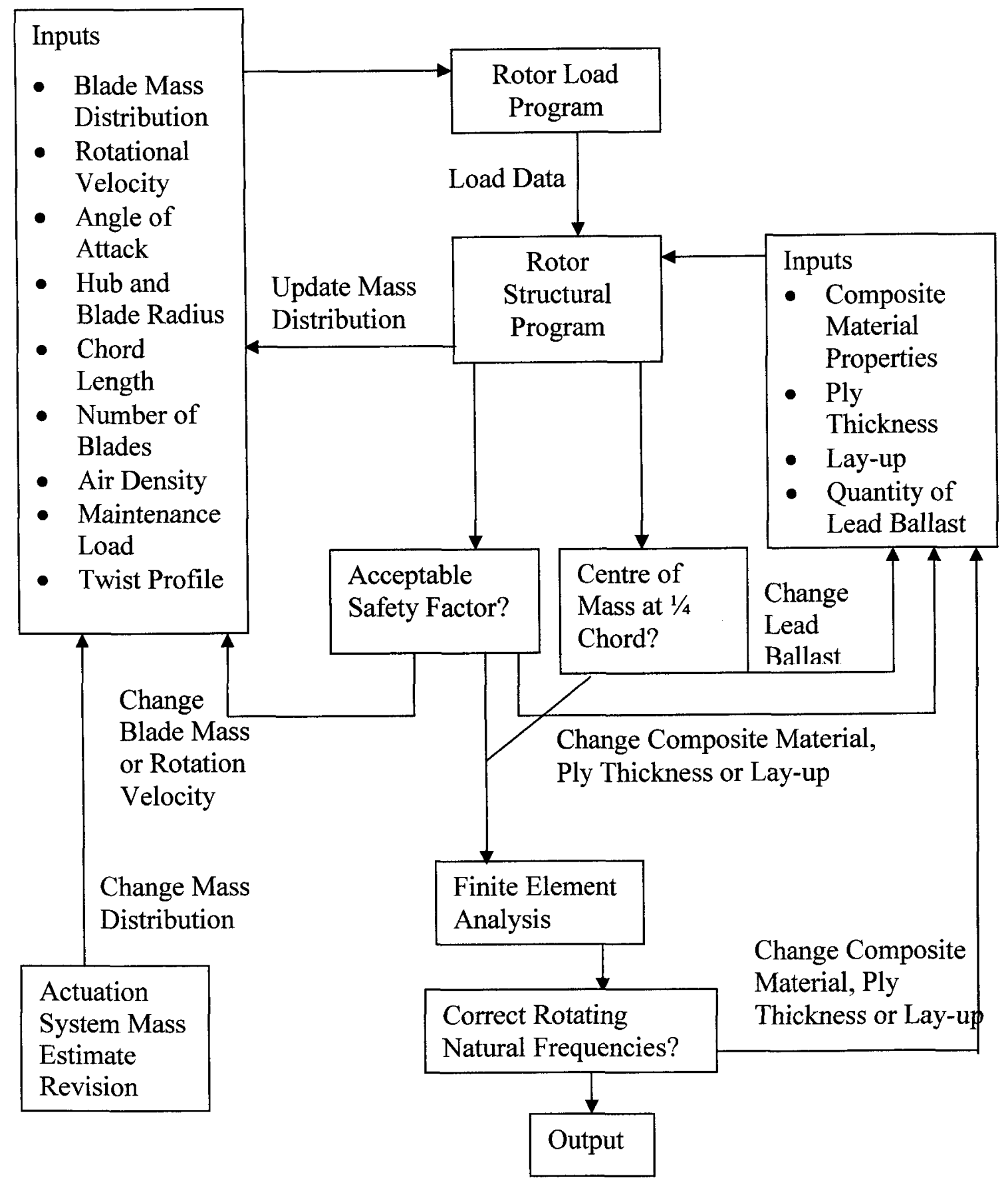

A 


\section{Appendix B: Drag Polar for NACA 0012 Airfoil [Abbott and Doenhoff 1959]}

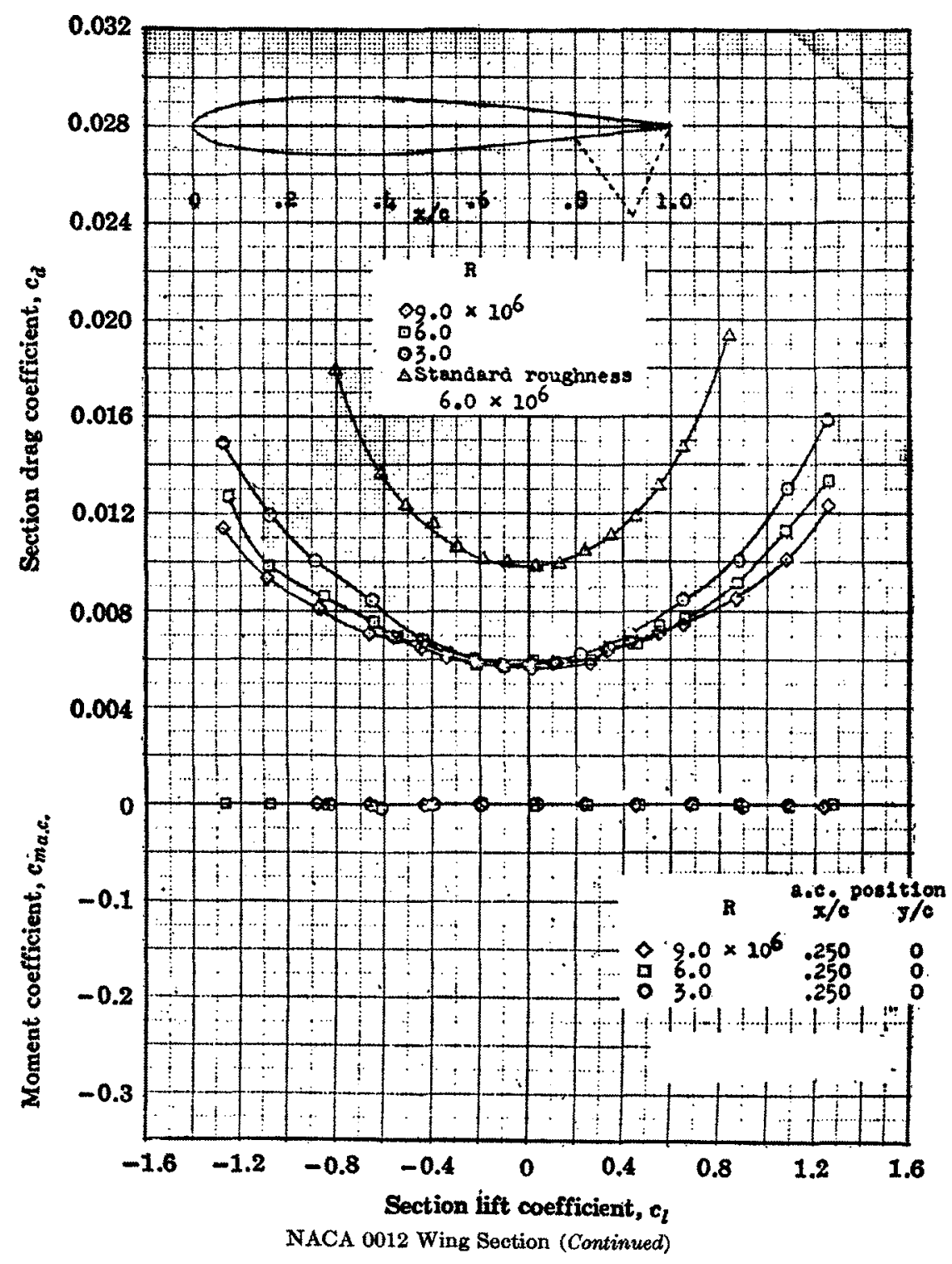




\section{Appendix C: Lift Data for NACA 0012 Airfoil [Abbott and Doenhoff 1959]}

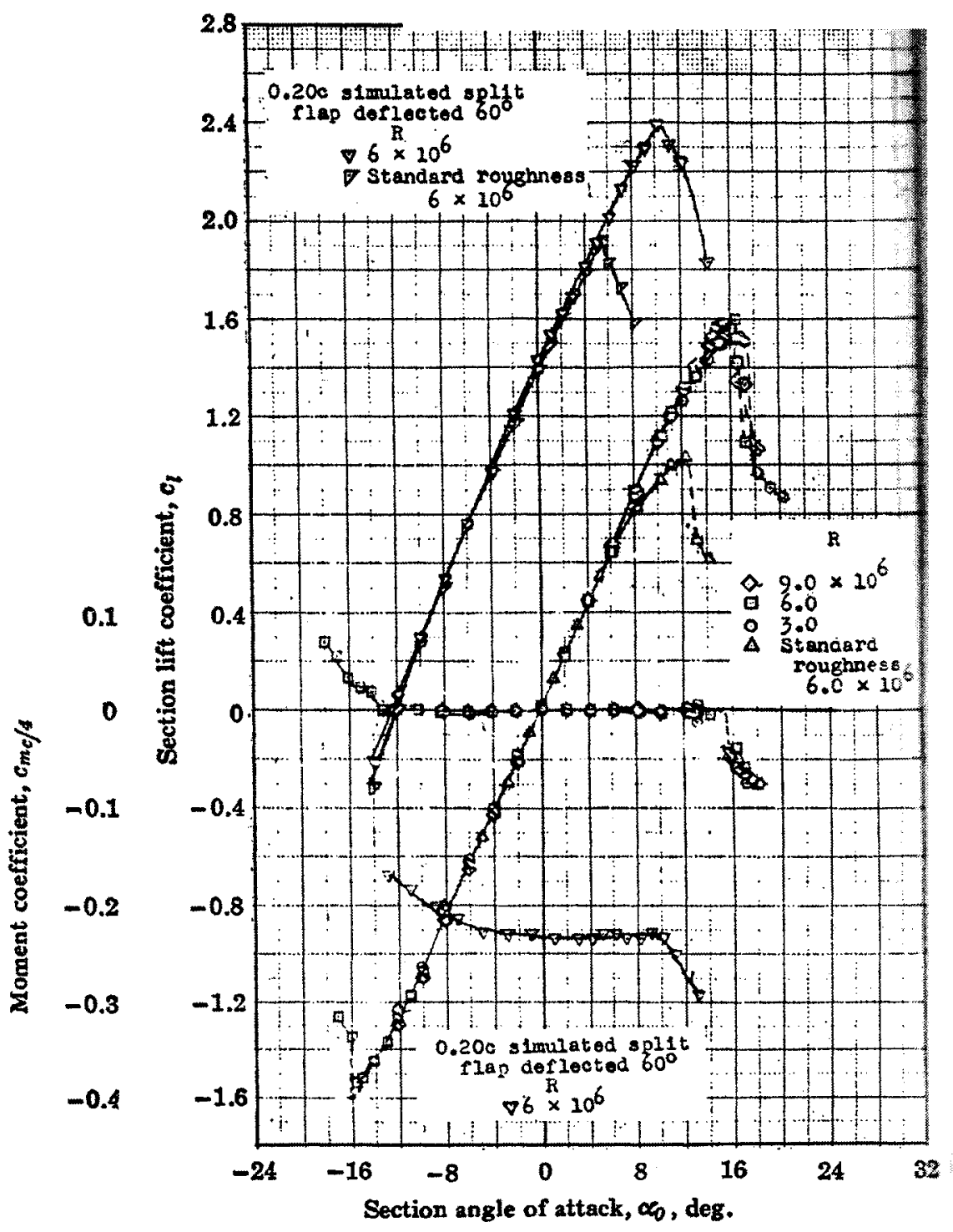

NACA 0012 Wing Section 

1959]

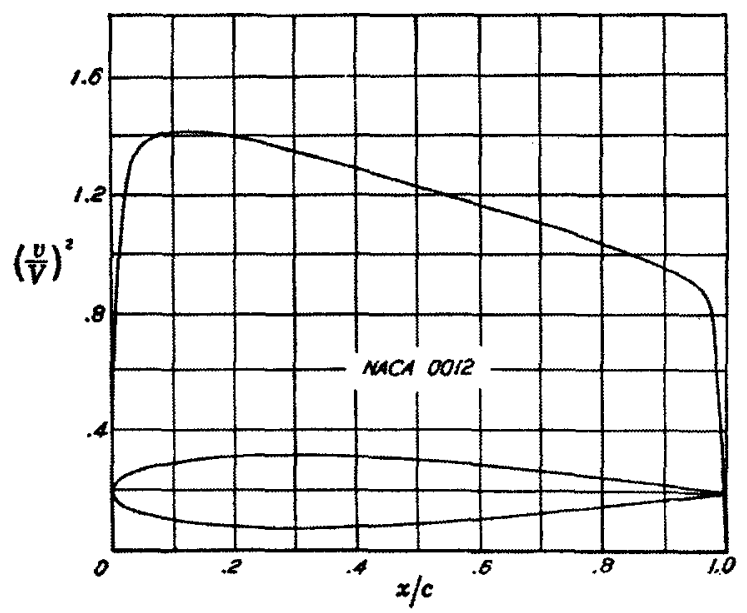

\begin{tabular}{|c|c|l|l|l|}
\hline $\begin{array}{c}x \\
\text { (per cent } c \text { ) }\end{array}$ & $\begin{array}{c}y \\
\text { (per cent } c \text { ) }\end{array}$ & \multicolumn{1}{|c|}{$(v / V)^{2}$} & $v / V$ & $\Delta v_{a} / V$ \\
\hline 0 & 0 & 0 & 0 & 1.988 \\
0.5 & $\ldots$. & 0.640 & 0.800 & 1.475 \\
1.25 & 1.894 & 1.010 & 1.005 & 1.199 \\
2.5 & 2.615 & 1.241 & 1.114 & 0.934 \\
5.0 & 3.555 & 1.378 & 1.174 & 0.685 \\
7.5 & 4.200 & 1.402 & 1.184 & 0.558 \\
10 & 4.683 & 1.411 & 1.188 & 0.479 \\
15 & 5.345 & 1.411 & 1.188 & 0.381 \\
20 & 5.737 & 1.399 & 1.183 & 0.319 \\
25 & 5.941 & 1.378 & 1.174 & 0.273 \\
30 & 6.002 & 1.350 & 1.162 & 0.239 \\
40 & 5.803 & 1.288 & 1.135 & 0.187 \\
50 & 5.294 & 1.228 & 1.108 & 0.149 \\
60 & 4.563 & 1.166 & 1.080 & 0.118 \\
70 & 3.664 & 1.109 & 1.053 & 0.092 \\
80 & 2.623 & 1.044 & 1.022 & 0.068 \\
90 & 1.448 & 0.956 & 0.978 & 0.044 \\
95 & 0.807 & 0.906 & 0.952 & 0.029 \\
100 & 0.126 & 0 & 0 & 0 \\
\hline \multicolumn{5}{|l}{} \\
\hline
\end{tabular}

NACA 0012 Basic Thickness Form 


\section{Appendix E: BO 105 Rotor Blade Fan Plot [Anonymous 1993]}

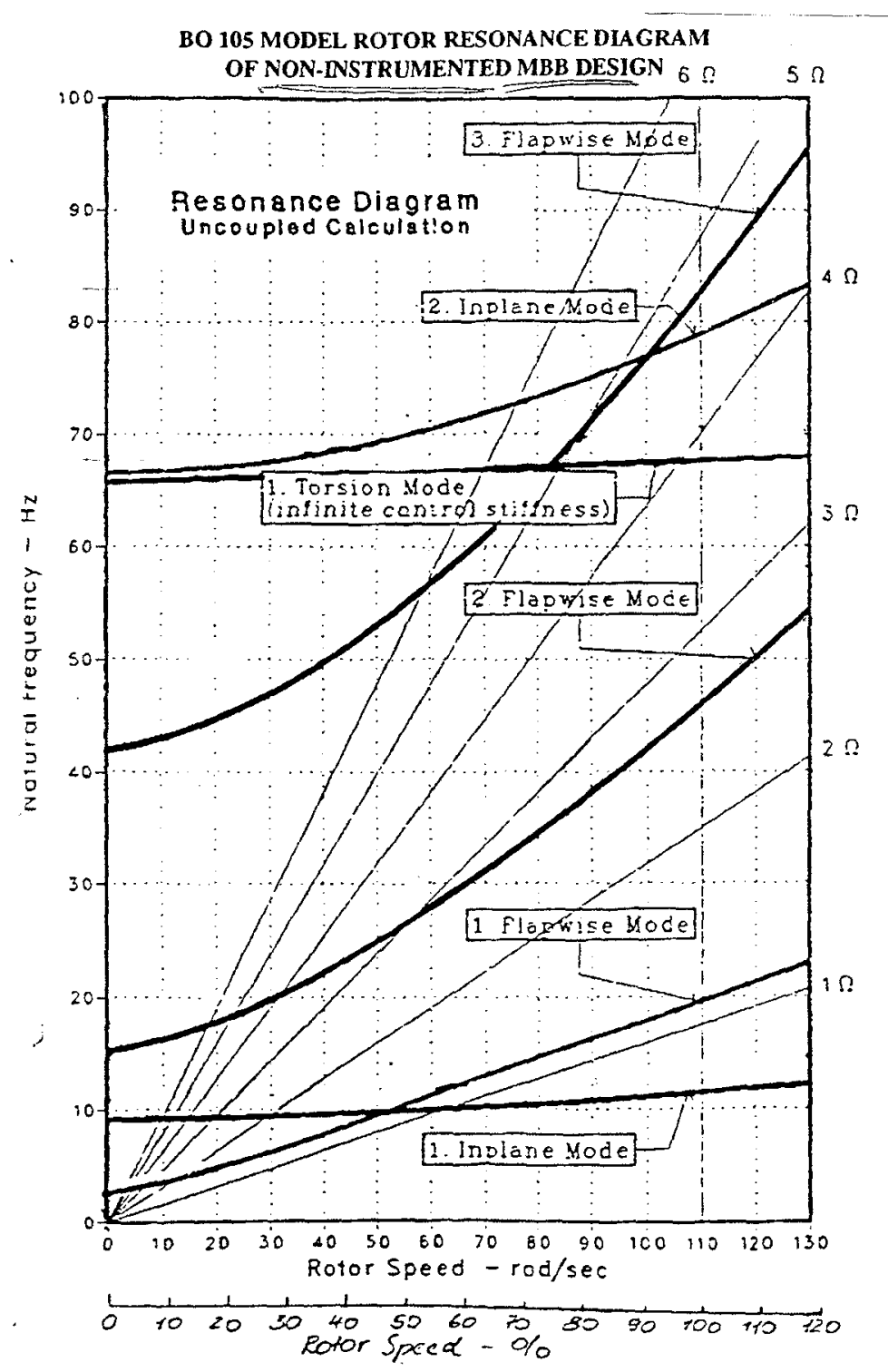

Reproduced with permission of the copyright owner. Further reproduction prohibited without permission. 